\title{
Homogenisation on homogeneous spaces
}

\author{
By Xue-Mei LI \\ Appendix by Dmitriy Rumynin
}

(Received June 11, 2016)

(Revised July 30, 2016)

\begin{abstract}
Motivated by collapsing of Riemannian manifolds and inhomogeneous scaling of left invariant Riemannian metrics on a real Lie group $G$ with a sub-group $H$, we introduce a family of interpolation equations on $G$ with a parameter $\epsilon>0$, interpolating hypo-elliptic diffusions on $H$ and translates of exponential maps on $G$ and examine the dynamics as $\epsilon \rightarrow 0$. When $H$ is compact, we use the reductive homogeneous structure of Nomizu to extract a converging family of stochastic processes (converging on the time scale $1 / \epsilon$ ), proving the convergence of the stochastic dynamics on the orbit spaces $G / H$ and their parallel translations, providing also an estimate on the rate of the convergence in the Wasserstein distance. Their limits are not necessarily Brownian motions and are classified algebraically by a Peter-Weyl's theorem for real Lie groups and geometrically using a weak notion of the naturally reductive property; the classifications allow to conclude the Markov property of the limit process. This can be considered as "taking the adiabatic limit" of the differential operators $\mathcal{L}^{\epsilon}=(1 / \epsilon) \sum_{k}\left(A_{k}\right)^{2}+(1 / \epsilon) A_{0}+Y_{0}$ where $Y_{0}, A_{k}$ are left invariant vector fields and $\left\{A_{k}\right\}$ generate the Lie-algebra of $H$.
\end{abstract}

\section{Introduction.}

By deforming the fibres of the Hopf fibration, the canonical round metric on the Lie group $S^{3}$ gives rise to a family of left invariant Berger's metrics which we denote by $m_{\epsilon}$. As $\epsilon$ approaches zero, the Riemannian manifolds $\left(S^{3}, m_{\epsilon}\right)$ converge to the lower dimensional two sphere $S^{2}(1 / 2)$ of radius $1 / 2$, while keeping the sectional curvatures bounded. See Cheeger and Gromov [13], Fukaya [25], and Kasue and Kumura [38] for other types of convergences of Riemannian manifolds. Let us denote by $\Delta_{S^{3}}^{\epsilon}$ and $\Delta_{S^{1}}$ the Laplacians on $\left(S^{3}, m_{\epsilon}\right)$ and on $S^{1}$ respectively, and also denote by $\Delta^{h}$ the horizontal Laplacian identified with the Laplacian on $S^{2}(1 / 2)=S^{3} / S^{1}$. These operators commute and $\Delta_{S^{3}}^{\epsilon}=(1 / \epsilon) \Delta_{S^{1}}+\Delta^{h}$. If $\left\{X_{1}, X_{2}, X_{3}\right\}$ are the Pauli matrices, identified with left invariant vector fields, then $\Delta_{S^{1}}=\left(X_{1}\right)^{2}, \Delta^{h}=\left(X_{2}\right)^{2}+\left(X_{3}\right)^{2}$. As $\epsilon$ approaches 0 , any eigenvalues of the Laplacian $\Delta_{S^{3}}^{\epsilon}$ coming from a non-zero eigenvalue of $(1 / \epsilon) \Delta_{S^{1}}$ is pushed to the back of the spectrum and an eigenfunction of $\Delta_{S^{3}}^{\epsilon}$, not constant in the fibre, flies away. In other words the spectrums of $S^{3}$ converge to that of $S^{2}$, see Tanno [67], Bérard-Bergery and Bourghignon [3], Urakawa [68] for discussions on the spectrum

2010 Mathematics Subject Classification. Primary 60Gxx, 60Hxx, 58J65, 58J70.

Key Words and Phrases. stochastic averaging, diffusion creation, adiabatic limit, Hörmander's conditions, classification of effective dynamics. 
of Laplacians on spheres, on homogeneous Riemannian manifolds and on Riemannian submersions with totally geodesic fibres.

Another interesting family of operators is $\left\{\gamma \Delta_{S^{1}}+\delta Y_{0}: \gamma>0, \delta>0\right\}$ where $Y_{0}=a X_{2}+b X_{3}$ is a non-zero unit length left invariant vector field. If $\gamma=0$, the time evolutions associated with the operators are geodesics on $S^{2}$; if $\delta=0$ the time evolutions with initial values in $H$ are scaled Brownian motions on $H$. Let us take $\gamma \rightarrow \infty$ and keep $\delta=1$. In other words we consider $\mathcal{L}^{\epsilon}=(1 / \epsilon) \Delta_{S^{1}}+Y_{0}$ where $\epsilon>0$. Unlike $\Delta_{S^{3}}^{\epsilon}$, the summands of $L^{\epsilon}$ do not commute, it is nevertheless easy to see that the orbits of the corresponding random evolutions exhibit interesting asymptotics. For this observe that the Hopf map on the Hopf fibration is a conservation law, this allows us to construct a converging family of first order random linear differential operators $\tilde{\mathcal{L}}^{\epsilon}$ (the evaluations of the Hopf map of the evolutions of $L^{\epsilon}$ and $\tilde{\mathcal{L}}^{\epsilon}$ agree) such that their time evolutions converge to a Markov process with effective limit $\lambda \Delta^{h}$ for an explicit constant $\lambda$. See Example 11.1, and also see $\mathrm{Li}[\mathbf{4 5}]$, where a related family of operators were studied. We study such phenomena on homogeneous manifolds.

Let $G$ be a smooth connected real Lie group, not necessarily compact, with a nontrivial closed proper subgroup $H$. Denote by $\mathfrak{g}$ and $\mathfrak{h}$ their respective Lie algebras. We assume that $H$ is connected, and compact for certain type of results and in this introduction. If $H$ is compact, there exists an $\mathrm{Ad}_{H}$-invariant inner product on $\mathfrak{g}$ which descends to $T_{o} M$ and induces a $G$-invariant Riemannian metric on $M$.

For a positive real number $\epsilon$, let us scale the inner product on $\mathfrak{h}$ by a number $\epsilon>0$. Let $\left\{X_{1}, \ldots, X_{p}, X_{p+1}, \ldots, X_{n}\right\}$ be an orthonormal basis of $\mathfrak{g}$ where $\left\{X_{j}, j \leq p\right\}$ is a basis of $\mathfrak{h}$. By declaring the left invariant vector fields $\left\{(1 / \sqrt{\epsilon}) X_{1}, \ldots,(1 / \sqrt{\epsilon}) X_{p}, X_{p+1}\right.$, $\left.\ldots, X_{n}\right\}$ an orthonormal frame we obtain a family of Riemannian metrics. Let us consider the sum of the squares of vector fields operators, arising from the non-homogeneous scaling,

$$
\mathcal{L}^{\epsilon}=\frac{1}{\epsilon} \mathcal{L}_{0}+Y_{0}, \quad \text { where } \quad \mathcal{L}_{0}=\frac{1}{2} \sum\left(A_{k}\right)^{2}+A_{0},
$$

$\left\{A_{k} \in \mathfrak{h}\right\}$ are bracket generating left invariant vector fields, and $Y_{0}$ is a left invariant vector field to be specified later. Similar to the Hopf fibration, a family of interpolation equations can be associated with $\mathcal{L}_{0}$ and $Y_{0}$. We study the asymptotics of the family of operators $\mathcal{L}^{\epsilon}$ on $[0,1]$ and on $[0,1 / \epsilon]$. Observe that $\mathcal{L}_{0}$ is not necessarily symmetric nor hypoelliptic in $G$.

We first consider $\mathcal{L}^{\epsilon}$ as a perturbation to the hypoelliptic diffusions induced by $(1 / \epsilon) \mathcal{L}_{0}$. The hypoelliptic diffusion processes stay in the orbits/fibres of their initial values while the perturbation in $Y_{0}$ direction induces motions transversal to the fibres, describing a conservation law. Our first task is to use it to understand the nature of the perturbation and to extract from them a family of first order random differential operators, $\tilde{\mathcal{L}}^{\epsilon}$, called the 'slow motions'. If the slow motions converge to a fixed point, we study the dynamics on the longer time scale $[0,1 / \epsilon]$. On the Riemannian homogeneous manifold, for $Y_{0}$ appropriately chosen, the 'effective limit' on $[0,1]$ is either a non-degenerate ODE or a fixed point. In the latter case and on the scale of $1 / \epsilon$, we would however treat the term involving $\mathcal{L}_{0}$ as a perturbation. It is counter intuitive to consider the dominate part as perturbation to the smaller part, but the perturbation, although very large in magnitude, 
is fast oscillating. The large oscillating motion will be averaged out, leaving an effective stochastic motion corresponding to a second order differential operator on $G$.

The singular perturbation problem described above can be considered as a stochastic homogenisation problem for the following family of stochastic differential equations (SDEs) on $G$,

$$
d g_{t}^{\epsilon}=\frac{1}{\sqrt{\epsilon}} \sum_{k=1}^{N} A_{k}\left(g_{t}^{\epsilon}\right) \circ d b_{t}^{k}+\frac{1}{\epsilon} A_{0}\left(g_{t}^{\epsilon}\right) d t+Y_{0}\left(g_{t}^{\epsilon}\right) d t, \quad g_{0}^{\epsilon}=g_{0},
$$

where $\circ$ denotes Stratonovich integrals. These SDEs belong to a family of equations, see Section 3, which interpolate between translates of one parameter subgroups of $G$ and hypoelliptic diffusion on $H$. Scaled by $1 / \epsilon$, the Markov generator of $\left(g_{t / \epsilon}^{\epsilon}\right)$ is precisely $(1 / \epsilon) \mathcal{L}^{\epsilon}$.

One of our tools is a reductive structure, in the sense of Nomizu [57], i.e. an $\mathrm{Ad}_{H^{-}}$ invariant sub-space $\mathfrak{m}$ of $\mathfrak{g}$ complementing $\mathfrak{h}$, not in the sense of having a completely irreducible adjoint representation. Such a complement $\mathfrak{m}$ exists if $H$ is compact, see Section 2 for further discussion where a non-Riemannian example is also given. If $Y_{0}$ belongs to a non-trivial irreducible invariant subspace $\mathfrak{m}_{k}$ of $\mathfrak{m}$, the time scale for taking the limit is determined by $\mathfrak{m}_{k}$, see Sections 4-6.2. Indeed, the effective limits can be classified using an orthogonal decomposition of $\mathfrak{m}$ into invariant subspaces, see Sections 7 10.

The reductive structure allows us to use the projection $\pi: G \rightarrow G / H$ as a 'conservation law', the projected process is a slow variable on the orbit space, and $\left(g_{t}^{\epsilon}\right)$ is a perturbation to the conservation law and we expect that the variable in the orbit space converge over a long time scale (equation (1.1) is already on this large time scale. To separate the slow and the fast variables, we 'horizontally lift back' the projected curves and obtain a slow variable on the total space $G$, the fast variable is a diffusion process on the subgroup $H$. The 'horizontal lifts' of the projected processes are random smooth curves driven by random ODEs whose generator will be denoted by $\tilde{\mathcal{L}}^{\epsilon}$, for almost surely all sample paths they are parallel translations along the projection. It is easy to see that they converge to the solution of an ODE: this is the averaging principle. We then identify those $Y_{0}$ for which the limiting ODE is trivial and in particular the projections of their solution curves are fixed points in the orbit space $G / H$. For such $Y_{0}$ we study the dynamics in a longer time interval and conclude that the family of random differentiable curves, determined by the family of random ODEs, converge to the sample paths of a non-trivial Markov process, i.e. diffusion reaction, the proof for this convergence is based on a theorem in [47]; we do however need to take care of the non-compactness of $G$ which is not entirely covered by [47]. In terms of the stochastic processes on the orbit manifolds, we prove their convergence on the interval of size $[0, \mathbf{1} / \epsilon]$ together with the convergence of their horizontal lifts, i.e. parallel translations along them converge to the stochastic parallel translation along the limiting diffusion process. The question whether or not the latter is a Markov process, on its own, will also be studied. The convergence will be in terms of the probability distributions induced by $(\mathbf{1} / \epsilon) \tilde{\mathcal{L}}_{\epsilon}$ in the weak topology and Wasserstein distance on the space $C([0,1] ; G)$ of continuous paths over $G$ and over $G / H$, see Section 6 , where the rate of convergence in the Wasserstein distance for their 
evaluations at specific times is given.

A heuristic argument based on multi-scale analysis, which we give shortly, appears to suggest the following centering condition: $\int_{H} Y_{0} F_{0} d h=0$ where $F_{0}$ is a solution to the effective parabolic equation and where $d h$ is the right invariant Haar measure on $H$. This is not quite the right assumption, we will assume instead that $Y_{0} \neq 0$, $\bar{Y}_{0} \equiv \int_{H} \operatorname{Ad}(h)\left(Y_{0}\right) d h=0$. Indeed, we show that $(\mathbf{1} / \epsilon) \tilde{\mathcal{L}}_{\epsilon}=\operatorname{Ad}\left(h_{t / \epsilon}(\omega)\right)\left(Y_{0}\right)$, where $\left(h_{t}\right)$ is a stochastic process in $H$; and that $\bar{Y}_{0}=0$ precisely when $Y_{0}$ is orthogonal to $\mathfrak{h} \oplus \mathfrak{m}_{0}$, where $\mathfrak{m}_{0}$ is the set of $\operatorname{Ad}_{H}$-invariant vectors in $\mathfrak{m}$ (Section 6.2 ).

\section{The heuristic Argument.}

Let us give the heuristics argument, made rigorous in Sections 4-6. For simplicity take $A_{0}=0$ and denote by $d h$ the normalised Haar measure on a compact $H$. Let $\mathcal{L}_{0}=(1 / 2) \sum_{k=1}^{p}\left(A_{k}\right)^{2}$ and let $F: \mathbb{R}_{+} \times G \rightarrow \mathbb{R}$ be a solution to the evolution equation $\partial F / \partial t=\mathcal{L}^{\epsilon} F$. Expand $F$ in $\epsilon, F(t)=F_{0}(t)+\epsilon F_{1}(t)+\epsilon^{2} F_{2}(t)+o\left(\epsilon^{2}\right)$, and plug this into the parabolic equation. Equating coefficients of $\epsilon^{0}$ and $\epsilon^{-1}$ we obtain the following equations:

$$
\mathcal{L}_{0} F_{1}+Y_{0} F_{0}=0, \quad \frac{\partial F_{0}}{\partial t}=Y_{0} F_{1}+\mathcal{L}_{0} F_{2}
$$

If $Y_{0} F_{0}$ averages to zero, the formal solution to the first equation is $F_{1}=-\mathcal{L}_{0}^{-1}\left(Y_{0} F_{0}\right)$. We should interpret this as an equation on $H$, and will solve this equation explicitly. The second equation reduces to $\partial F_{0} / \partial t=-Y_{0}\left(\mathcal{L}_{0}^{-1}\left(Y_{0} F_{0}\right)\right)+\mathcal{L}_{0} F_{2}$ which we integrate with respect to $d h$. Since $\mathcal{L}_{0}$ is symmetric by Lemma 5.1 and $\mathcal{L}_{0} F_{2}$ averages to zero. We obtain the equation for the effective motion:

$$
\frac{d}{d t} \int_{H} F_{0} d h=-\int_{H} Y_{0} \mathcal{L}_{0}^{-1}\left(Y_{0} F_{0}\right) d h .
$$

The computations are formal. Firstly we neglected higher powers of $1 / \epsilon$, which are very large for $\epsilon$ small. Secondly we assumed that the Poisson equation $\mathcal{L}_{0}=Y_{0} F_{0}$ is solvable. Finally we assumed that $\int_{H} \mathcal{L}_{0} F_{2} d h=0$. Observing that $\mathcal{L}_{0}$ is not hypoelliptic on $G$, we must reduce the system to a space where it is, to justify these assertions.

\section{Main result.}

We assume that $\mathfrak{m}$ is a reductive structure in the sense of Nomizu, a list of useful reductive decompositions are given in Section 2. Denote by $\operatorname{Ad}_{H}: H \rightarrow \mathbb{L}(\mathfrak{g} ; \mathfrak{g})$ the restriction of the adjoint of $G$ to $H$ and also the restricted representation $\operatorname{Ad}_{H}: H \rightarrow$ $\mathbb{L}(\mathfrak{m} ; \mathfrak{m})$. Denote by $\mathfrak{m}_{0} \subset \mathfrak{m}$ the space of invariant vectors of $\operatorname{Ad}_{H}$. Let $\mathfrak{m}=\mathfrak{m}_{0} \oplus \mathfrak{m}_{1} \oplus$ $\cdots \oplus \mathfrak{m}_{l}$ be an invariant decomposition for $\operatorname{Ad}_{H}$ where, for each $l \neq 0, \mathfrak{m}_{l}$ is an irreducible invariant space. If $H$ is compact we may and will assume that the decomposition is orthogonal. The main results are in three parts.

(1) Separation of slow and fast variables and Reduction. Use the Ehresman connection on the principal bundle $\pi: G \rightarrow G / H$, determined by $\mathfrak{m}$, and horizontal lifts of curves from $G / H$ to $G$ we deduce stochastic equations for the horizontal lifts $\tilde{x}_{t}^{\epsilon}$ of $x_{t}^{\epsilon}=\pi\left(g_{t}^{\epsilon}\right)$, the slow motions, and for the fast motions $h_{t}^{\epsilon}$ on $H$. 
(2) Convergence of the slow components. If $Y_{0}$ is orthogonal to $\mathfrak{m}_{0}$ and $\left\{A_{0}, A_{1}, \ldots\right.$, $\left.A_{N}\right\}$ generates $\mathfrak{h}$, the stochastic processes $\left(\tilde{x}_{t / \epsilon}^{\epsilon}, t \in[0, T]\right)$ and $\left(x_{t / \epsilon}^{\epsilon}, t \in[0, T]\right)$ converge weakly, as $\epsilon \rightarrow 0$, to $\bar{u}_{t}$ and $\bar{x}_{t}$ respectively, in the weak topology on the path space over $G$ and in Wasserstein distance. A rate of convergence is given.

(3) Effective Process. Assume $A_{0}=0$ and $Y_{0} \in \mathfrak{m}_{l}$. The effective process on $G$ is a Markov process with generator $c \Delta_{m_{l}}$. If furthermore $M$ is isotropy irreducible and if $M$ is naturally reductive (and more generally) then $\bar{x}_{t}$ is a scaled Brownian motion whose scale is computable.

We indicate the problems pertinent to part (3). The reduced first order random differential operators give rise to second order differential operators by the action of the Lie brackets. If the Lie bracket between $A_{k}$ and $Y_{0}$ is not trivial, randomness is generated in the $\left[A_{k}, Y_{0}\right]$ direction and transmitted from the vertical to the horizontal directions. We ask the question whether the limiting operators are scaled horizontal Laplacians. This is so for the Hopf fibration. However it only takes a moment to figure out this cannot be always true. The noise cannot be transmitted to directions in an irreducible invariant subspace of $\mathfrak{m}$ not containing $Y_{0}$. If $\mathfrak{m}_{l}$ is the irreducible invariant subspace containing $Y_{0}$, the action of $\operatorname{Ad}_{H}$ generates directions in $\mathfrak{m}_{l}$, not any direction in a component complementary to $\mathfrak{m}_{l}$ and so the rank of $\overline{\mathcal{L}}$ is at most $\operatorname{dim}\left(\mathfrak{m}_{l}\right)$. Within an irreducible $\operatorname{Ad}_{H}$-invariant subspace $\mathfrak{m}_{l}$, the transmission of the noise should be 'homogeneous' and we might expect that there is essentially only one, up to scalar multiplication, candidate effective second order differential operator: the generalised Laplacian 'operator' $\Delta_{\mathfrak{m}_{l}}:=$ $\sum L_{Y_{i}} L_{Y_{i}}$ where $\left\{Y_{i}\right\}$ is an orthonormal basis of $\mathfrak{m}_{l}$. The generalised Laplacian $\Delta_{\mathfrak{m}_{l}}$ is independent of the choice of the basis.

If $\left\{A_{1}, \ldots, A_{p}\right\}$ is a basis of $\mathfrak{h}$, we solve a Poisson equation and prove that $\overline{\mathcal{L}}=$ $\sum_{i, j} a_{i, j}\left(Y_{0}\right) L_{Y_{i}} L_{Y_{j}}$, where $\left\{Y_{i}\right\}$ is a basis of the irreducible $\operatorname{Ad}_{H}$ invariant space $\mathfrak{m}_{l}$ containing $Y_{0}$ and $a_{i, j}\left(Y_{0}\right)$ trigonometric functions of the adjoint sub-representation in this basis. It can be written in terms of eigenvalues of $\mathcal{L}_{0}$, computable from the 'Casimir', and has an $\mathrm{Ad}_{H}$-invariant form. If $Y_{0} \in \mathfrak{m}_{l}$ then by the real Peter-Weyl theorem, a proof of which by Dmitriy Rumynin is appended at the end of the paper, we see that $\overline{\mathcal{L}}=\left(1 / \operatorname{dim}\left(\mathfrak{m}_{l}\right) \lambda_{l}\right)\left|Y_{0}\right|^{2} \Delta_{\mathfrak{m}_{l}}$. In case $G$ is unimodular and $\mathfrak{m}$ is irreducible this is the 'horizontal Laplacian'. If $\left\{A_{k}\right\}$ is only a set of generators of the Lie algebra $\mathfrak{h}$, a similar formula holds with the constant $\lambda_{l}$ replaced by a constant $\lambda\left(Y_{0}\right)$, depending on $Y_{0}$ in general.

The above descriptions are algebraic, in Section 10 we discuss their differential geometric counterpart. Let $G$ be endowed with a left invariant and $\operatorname{Ad}_{H}$-invariant Riemannian metric and let $M=G / H$ be given the induced $G$-invariant Riemannian metric. The translates of the one parameter subgroups of $G$ are not necessarily geodesics for the Levi-Civita connection on $G$. Their projections to the Riemannian homogeneous manifold are not necessarily geodesics either. In general $\overline{\mathcal{L}}$ needs not be the Laplace-Beltrami operator even if it is elliptic for it may have a nontrivial drift. The more symmetries there are, the closer is the effective diffusion to a Brownian motion. A maximally symmetric and non-degenerate effective process ought to be a scaled Brownian motion. In other words we like to see the convergence of the random smooth curves in the orbit manifold to a scaled Brownian motion, which will be studied under the condition on the 
trace of $\operatorname{ad}(Z)$, under which $\operatorname{trace}_{\mathfrak{m}_{l}} \nabla^{L} d=\operatorname{trace}_{\mathfrak{m}_{l}} \nabla d$ and $\operatorname{trace}_{\mathfrak{m}_{l}} \nabla^{c} d=$ trace $_{\mathfrak{m}_{l}} \nabla d$ on $M$, where $\nabla$ denotes the Levi-Civita connections on $G$ and on $M$ and $\nabla^{c}$ denotes the canonical connection on $M$.

\section{The context.}

The study of parabolic differential equation of the type $\partial / \partial t=Y_{0}+(1 / \epsilon) \mathcal{L}_{0}$ where $\mathcal{L}_{0}$ is an elliptic operator and $Y_{0}$ a vector field, in the non-geometric settings goes back to Smoluchowski (1916) and to Kramers (1940) [41] and are known as SmoluchowskiKramers limits. This was taken up by Nelson [56] for unifying Einstein's theory and Ornstein-Uhlenbeck's theory of Brownian motions. The further scaling by $1 / \epsilon$, leading to the asymptotic problems for $(1 / \epsilon) Y_{0}+\left(1 / \epsilon^{2}\right) \mathcal{L}_{0}$, are known by the a number of terminologies in a great many subjects: averaging principle, stochastic homogenisation, multi-scale analysis, singular perturbation problem, or taking the adiabatic limit. They also appear in the study of interacting particle systems. We treat our problem as a perturbation to a non conventional conserved system and use techniques from multi-scale analysis and stochastic homogenisation. For perturbation to Hamiltonian systems see Freidlin [22], see also Freidlin and Weber [23]. The use of 'Hamiltonian' is quite liberal, by which we merely mean that they are conserved quantities, here they are manifold valued functions or orbits. Some ideas in this paper were developed from Li [45]. Convergences of Riemannian metrics from the probabilistic point of view, have been studied by Ikeda and Ogura [35] and Ogura and Taniguchi [59].

Our reduced equations are ODEs on manifolds with random coefficients, the study of random ODEs on $\mathbb{R}^{n}$ goes back to Stratonovich [66], Khasminski [28], Kohler and Papanicolaou [60], Papanicolaou and Varadhan [61] and Borodin and Freidlin [11]. The convergence from operator semigroup point of view is studied by Kurtz [42]. A collection of limit theorems, some of which are used here, together with a set of more complete references can be found by $\mathrm{Li}[\mathbf{4 7}]$. For stochastic averaging with geometric structures, we refer to $\mathrm{Li}[\mathbf{4 4}]$ for a stochastic integrable Hamiltonian systems in symplectic manifolds, and to Gargate and Ruffino [26] and Hogele and Ruffino [31] for studies of stochastic flows on foliated manifolds.

We also use multi-scale analysis to separate the slow and fast variables and draw from the work by Elworthy, LeJan and Li [20]. Our reduction in complexity bears resemblance to aspects of geometric mechanics and Hamiltonian reduction, see Lázaro-Camí and Ortega [43], also Holm, Marsden, Ratiu and Weinstein [32], and Marsden, Misiolek, Ortega, Perlmutter and Ratiu [51]. The problem of identifying limit operators is reminiscent of that in Freidlin and Wentzell [24] where an explicit Markov process on a graph was obtained from the level sets of a Hamiltonian function in $\mathbb{R}^{2}$, but they are obviously very different in nature.

The asymptotics of linear operators of the form $(1 / \epsilon) \mathcal{L}^{\epsilon}:=\left(1 / \epsilon^{2}\right) \mathcal{L}_{0}+(1 / \epsilon) \mathcal{L}_{1}$ are often referred as taking the adiabatic limits in some contexts, see for example Mazzeo and Melrose [52] and Berline, Getzler and Vergne [5]. These operators act not just on scalar functions and their objectives are motivated by problems in topology. Motivated by analysis of loop spaces Bismut [8], [9] studied 'limits' of a family of hypoelliptic differential operators on the tangent bundle or the cotangent bundle of a compact Riemannian manifold $M$, see also Bismut and Lebeau [10] for an earlier work. They are devoted to study 
the convergence of probability distributions of the projections of hypoelliptic diffusions to Brownian motion. Unlike in these studies our state space is not necessarily compact, the operators $\mathcal{L}^{\epsilon}$ are not necessarily hypoelliptic, the projections of the effective limiting diffusions are not always Brownian motions. Classify the limits are one of our objectives. In another direction, Nelson [56], proved that the physical particles whose velocity are one dimensional Ornstein-Uhlenbeck processes approximates, in high temperature, one dimensional Brownian motions. This was generalised to manifolds by Dowell [15]. This study bears much resemblance to the above mentioned articles; however the model and the objectives in $\mathrm{Li}[\mathbf{4 6}]$ are much closer: SDEs on the orthonormal frame bundle are used to show that Brownian motions are generated by 'geodesics' with rapidly changing directions.

\section{Notation, preliminaries, and examples.}

If $g \in G$ we denote by $L_{g}$ and $R_{g}$ the left and right multiplications on $G$. Denote by $d L_{g}$ and $d R_{g}$ their differentials. The differentials are also denoted by $T L_{g}$ and $T R_{g}$ where these are traditionally used. Denote by Ad the adjoint representation of $G$ with $\operatorname{Ad}_{H}$ its restriction to $H$ and ad the adjoint representation on $\mathfrak{g}$ with restriction $\operatorname{ad}_{\mathfrak{h}}$. Both the notation $\operatorname{ad}_{X} Y$ and $[X, Y]$ are used. If $\mathfrak{m}$ is a subspace of $\mathfrak{g}, g \mathfrak{m}$ denotes the set of left translates of elements of $\mathfrak{m}$ to $T_{g} G$. We identify the set of left invariant vector fields with elements of the Lie algebra, denote them by the same letters or with the superscript $*$. If $V$ is a vector field and $f$ function, both $L_{V} f$ and $V f$ are use to denote Lie differentiation in the direction $V$, the latter for simplicity, the first to avoid confusing with operators denoted by $\mathcal{L}$. Also, $\Delta_{H}$ denotes the Laplacian for a bi-invariant metric on the Lie group $H$.

A manifold $M$ is homogeneous if there is a transitive action by a Lie group $G$. It can be represented as the coset space $G / H$ where $H$ is the isotropy group at a point $o$. The coset space is given the unique manifold structure such that $g H \in G / H \mapsto \bar{L}_{g} O \in M$ is a diffeomorphism where $\bar{L}_{g}$ denotes the action of $g$. If $\pi: G \rightarrow M$ denotes the projection taking $g$ to the coset $g H$ and $1 \in G$ the identity, the sub-Lie algebra $\mathfrak{h}$ is the kernel of $(d \pi)_{1}$ and $T_{o} M$ is isomorphic to a complement of $\mathfrak{h}$ in $\mathfrak{g}$.

The homogeneous space $G / H$ is reductive if in the Lie algebra $\mathfrak{g}$ there exists a subspace $\mathfrak{m}$ such that $\operatorname{Ad}(H)(\mathfrak{m}) \subset \mathfrak{m}$ and $\mathfrak{g}=\mathfrak{h} \oplus \mathfrak{m}$ is a vector space direct sum. By $\operatorname{Ad}_{H}(\mathfrak{m})$ we mean the image of $H$ under the adjoint map, treated as linear maps restricted to $\mathfrak{m}$. We say that $\mathfrak{g}=\mathfrak{h} \oplus \mathfrak{m}$ is a reductive decomposition and $(\mathfrak{g}, \mathfrak{h})$ a reductive pair. This implies that $[\mathfrak{h}, \mathfrak{m}] \subset \mathfrak{m}$ and vice versa if $H$ is connected. The homogeneous space is also called reductive, a reductive property in the sense of Nomizu, a concept different from a Lie group being reductive. In particular, the Lie group $G$ is not necessarily reductive, by which we mean its adjoint representation is completely reducible.

We discuss briefly the connectedness of the Lie group. Firstly if $H$ and $G / H$ are connected, so is $G$. This follows from the fact that a topological space $X$ is connected if and only if every continuous function from $X$ to $\{0,1\}$ is constant. The identity component $G^{0}$ of $G$ is a normal subgroup of $G$, and any other component of $G$ is a coset of $G^{0}$. The component group $G / G^{0}$ is discrete. If a Lie group $G$ acts transitively on a connected smooth manifold $M$, so does $G^{0}$. See [27]. Our stochastic processes are 
continuous in time, and hence we may and will assume that both $G$ and $H$ are connected.

The existence of an $\operatorname{Ad}_{H}$ invariant inner product is much easier than requesting an Ad-invariant inner product on $\mathfrak{g}$ which is equivalent to $G$ is of compact type. If $H$ is compact, by the unitary trick, there exists an $\operatorname{Ad}_{H^{-}}$-invariant inner product on $\mathfrak{g}$ and a reductive structure by setting $\mathfrak{m}=\mathfrak{h}^{\perp}$. The compactness of $H$ is not a restriction for a Riemannian homogeneous manifold. If $G / H$ is a connected Riemannian homogeneous space and $G$ is connected then by a theorem of van Danzig and van der Waerden (1928), the isotropy isometry groups at every point is compact, Kobayashi [39]. If $H$ is connected and its Lie algebra is reductive in $\mathfrak{g}$, in the sense that $\operatorname{ad}(\mathfrak{h})$ in $\mathfrak{g}$ is completely reducible, then $G / H$ is reductive. The Euclidean space example below will cover the averaging model used in Liao and Wang [49]. Let $G=E(n)$ be the space of rigid motions on $\mathbb{R}^{n}$,

$$
E(n)=\left\{\left(\begin{array}{cc}
R & v \\
0 & 1
\end{array}\right): R \in S O(n), v \in \mathbb{R}^{n}\right\}
$$

and $H$ its subgroup of rotations. Elements of $H$ fix the point $o=(0,1)^{T}, E(n) / H=$ $\left\{(x, 1)^{T}, x \in \mathbb{R}^{n}\right\}$ and a matrix in $E(n)$ projects to its last column. We may take

$$
\mathfrak{h}=\left\{\left(\begin{array}{cc}
A & 0 \\
0 & 0
\end{array}\right), A \in \mathfrak{s o}(n)\right\}, \quad \mathfrak{m}=\left\{\left(\begin{array}{ll}
0 & v \\
0 & 0
\end{array}\right), v \in \mathbb{R}^{n}\right\} .
$$

A reductive structure may not be unique. For example, let $G$ be a connected compact Lie group, $H=\{(g, g): g \in G\}$ and $\mathfrak{h}=\{(X, X): X \in \mathfrak{g}\}$. Then $G=(G \times G) / H$ is a reductive homogeneous space in three ways: $\mathfrak{m}^{0}=\{(X,-X), X \in \mathfrak{g}\}$,

$$
\mathfrak{m}^{+}=\{(0, X), X \in \mathfrak{g}\}, \quad \mathfrak{m}^{-}=\{(X, 0), X \in \mathfrak{g}\} .
$$

The first one, $\mathfrak{h} \oplus \mathfrak{m}^{0}$, is the symmetric decomposition.

A definite metric is not necessary either. If $\mathfrak{g}$ admits an $\operatorname{Ad}_{H}$ invariant nondegenerate bilinear form such that its restriction to $\mathfrak{h}$ is non-degenerate, let $\mathfrak{m}=\{Y \in$ $\mathfrak{g}: B(X, Y)=0, \forall X \in \mathfrak{h}\}$. In [40, Chapter 10], Kobayashi and Nomizu considered the case where $B$ is $\operatorname{Ad}(G)$ invariant. Their proof can be modified to work here.

\subsection{The Motivating Example.}

As a motivating example, we take $G=S U(2), H=U(1)$ and the bi-invariant Riemannian metric such that $\langle A, B\rangle=(1 / 2)$ trace $A B^{*}$ where $A, B \in \mathfrak{g}$. Let

$$
X_{1}=\left(\begin{array}{cc}
i & 0 \\
0 & -i
\end{array}\right), \quad X_{2}=\left(\begin{array}{cc}
0 & 1 \\
-1 & 0
\end{array}\right), \quad X_{3}=\left(\begin{array}{cc}
0 & i \\
i & 0
\end{array}\right)
$$

be the Pauli matrices. They form an orthonormal basis of the Lie algebra $\mathfrak{g}$ with respect to the bi-invariant metric and $G$ is the canonical three sphere in $\mathbb{R}^{4}$. The Lie algebra $\mathfrak{h}$ is generated by $X_{1}$ and we take $\mathfrak{m}$ to be the vector space generated by the remaining two Pauli matrices and obtain the family of Berger's metric, which we denote as before by $\left\{m^{\epsilon}, \epsilon>0\right\}$.

Let us first take the Brownian motions on Bergers' spheres. A Brownian motion on $\left(S^{3}, m^{\epsilon}\right)$ is determined by the Hörmander type operator $\Delta^{\epsilon}=\left(X_{1}\right)^{2} / \epsilon+\sum_{i=2}^{3}\left(X_{i}\right)^{2}$. 
Although no longer associated with the round metric, there are many symmetries in the following SDEs,

$$
d g_{t}=\frac{1}{\sqrt{\epsilon}} X_{1}\left(g_{t}\right) \circ d b_{t}^{1}+X_{2}\left(g_{t}\right) \circ d b_{t}^{2}+X_{3}\left(g_{t}\right) \circ d b_{t}^{3} .
$$

In particular the probability distributions of its slow components at time $t$ are independent of $\epsilon$, see Example 4.3 in Section 4. Breaking up the symmetry we may consider the equation,

$$
d g_{t}=\frac{1}{\sqrt{\epsilon}} X_{1}\left(g_{t}\right) \circ d b_{t}^{1}+X_{2}\left(g_{t}\right) \circ d b_{t}^{2},
$$

in which the incoming noise is 2-dimensional and its Markov generator satisfies the strong Hörmander's conditions. We will go one step further and use a one dimensional noise. Let $\left(b_{t}\right)$ be a real valued Brownian motion. If $Y_{0}=c_{2} X_{2}+c_{3} X_{3}$, where $c_{2}, c_{3}$ are numbers not simultaneously zero, the infinitesimal generator of the equation

$$
d g_{t}=\frac{1}{\sqrt{\epsilon}} X_{1}\left(g_{t}\right) \circ d b_{t}+\left(Y_{0}\right)\left(g_{t}\right) d t
$$

satisfies weak Hörmander's conditions. Indeed by the structural equations, $\left[Y_{0}, X_{1}\right]=$ $2 c_{2} X_{3}-2 c_{3} X_{2}$, and the matrix

$$
\left(\begin{array}{ccc}
\frac{1}{\sqrt{\epsilon}} & 0 & 0 \\
0 & c_{2} & -2 c_{3} \\
0 & c_{3} & 2 c_{2}
\end{array}\right)
$$

is not degenerate. In general, equation (1.1) need not satisfy Hörmander's condition. This example is concluded in Example 11.1, using Corollary 9.2.

\section{The interpolation equations.}

Given a left invariant Riemannian metric on $G$, we consider a family of nonhomogeneously scaled Riemannian metrics. To define these let $\left\{X_{1}, \ldots, X_{n}\right\}$ be an orthonormal basis of $\mathfrak{g}$ extending an orthonormal basis $\left\{X_{1}, \ldots, X_{p}\right\}$ of $\mathfrak{h}$ and let

$$
E^{\epsilon}=\left\{\frac{1}{\sqrt{\epsilon}} X_{1}^{*}, \ldots, \frac{1}{\sqrt{\epsilon}} X_{p}^{*}, X_{p+1}^{*}, \ldots X_{n}^{*}\right\} .
$$

The superscript $*$ above a letter denotes the corresponding left invariant vector field which we will omit, from time to time, in favour of simplicity. The dual frame of $E^{\epsilon}$ defines a family of left invariant Riemannian metrics on $G$ which are denoted by $m^{\epsilon}$, then $E^{\epsilon}$ is an orthonormal frame. In this article we are not concerned with the problem of keeping the sectional curvatures bounded, and $G$ needs not be compact.

Let $\left(\Omega, \mathcal{F}, \mathcal{F}_{t}, P\right)$ be a filtered probability space satisfying the usual assumptions and $\left(b_{t}^{k}, k=1, \ldots, N,\right)$ a family of independent real valued Brownian motions. Let $\gamma, \delta$ be positive real numbers, $X_{k} \in \mathfrak{h}$ as above, and $Y_{0} \in \mathfrak{g}$. We study the stochastic dynamics 
associated with the above inhomogeneous scalings of Riemannian metrics and propose the following interpolation equation that, in the limit of $\epsilon \rightarrow 0$, describes the 'effective motion' across the 'orbits',

$$
d g_{t}=\sum_{k=1}^{p} \gamma X_{k}\left(g_{t}\right) \circ d b_{t}^{k}+\delta Y_{0}\left(g_{t}\right) d t
$$

with a given initial value $g_{0}$. Here o denotes Stratonovich integration. Their solutions are Markov processes whose probability laws are determined by the fundamental solutions to the following parabolic equation $\partial / \partial t=(1 / 2) \gamma^{2} \sum_{k=1}^{p}\left(X_{k}\right)^{2}+\delta Y_{0}$.

For $\gamma=1$ and $\delta=1 /\left|Y_{0}\right|$, these equations are driven by unit length vector fields on the Riemannian manifold $\left(G, m^{\epsilon}\right)$. If $\delta=0$ the solution with its initial value the identity of the group is a scaled Brownian motion on the subgroup $H$. If $\gamma=0$, the solutions are translates of the one parameter family subgroup generated by $Y_{0}$. We denote the vector space generated by $\left\{X_{p+1}, \ldots, X_{n}\right\}$ by $\mathfrak{m}$. We assume in addition that $\mathfrak{m}$ is $\operatorname{Ad}_{H^{-}}$ invariant. If $Y_{0} \in \mathfrak{m}$, these one parameter families, $\exp \left(\delta t Y_{0}\right)$, are horizontal curves for the horizontal distribution determined by $\mathfrak{m}$. The solutions of (3.1) interpolate between translates of the one parameter group, generated by $\delta Y_{0}$, on $G$ and Brownian motion on $H$. The question is: if we take $\gamma \rightarrow \infty$ while keeping $\delta$ fixed, what can we say about the solutions of these equations?

We will work on more general operators, allowing $\left\{A_{k}\right\}$ to be a Lie algebra generating subset of $\mathfrak{h}$ instead of assuming ellipticity. Take $Y_{0} \in \mathfrak{m}$. We study the following family of SDEs, where $\epsilon>0$ is a small parameter,

$$
d g_{t}^{\epsilon}=\frac{1}{\sqrt{\epsilon}} \sum_{k=1}^{N} A_{k}\left(g_{t}^{\epsilon}\right) \circ d b_{t}^{k}+\frac{1}{\epsilon} A_{0}\left(g_{0}^{\epsilon}\right) d t+Y_{0}\left(g_{t}^{\epsilon}\right) d t, \quad g_{0}^{\epsilon}=g_{0} .
$$

The condition $g_{0}^{\epsilon}=g_{0}$, independent of $\epsilon$, is assumed only for the simplicity of the statements. Let $\mathcal{L}_{0}=(1 / 2) \sum\left(A_{k}\right)^{2}+A_{0}$. The corresponding parabolic problems are:

$$
\frac{\partial}{\partial t}=\frac{1}{\epsilon} \mathcal{L}_{0}+Y_{0}, \quad \frac{\partial}{\partial t}=\frac{1}{\epsilon^{2}} \mathcal{L}_{0}+\frac{1}{\epsilon} Y_{0}
$$

For intuition, let us review the theory for randomly perturbed Hamiltonian dynamics. If $H$ is a function on $R^{2 n}$, it is a first integral for the following Hamiltonian system: $\dot{q}=-\partial H / \partial p, \dot{p}=\partial H / \partial q$. Let $x^{\epsilon}(t):=\left(q^{\epsilon}(t), p^{\epsilon}(t)\right)$ denote solutions to a perturbed Hamiltonian system, which we do not specify, then $H^{\epsilon}(t):=H\left(p^{\epsilon}(t), q^{\epsilon}(t)\right)$ varies slowly with $t$ on $[0,1]$. Under suitable mixing conditions on the perturbation the stochastic processes $H^{\epsilon}(t / \epsilon)$ converge, see e.g. Freidlin and Wentzel [24] and Arnold [2]. Although we do not have a Hamiltonian system, the projection $\pi: G \rightarrow G / H$ is a conservation law for an 'unperturbed' dynamical system, which is the key for the reduction to the slow varying dynamics. If $\left(y_{t}^{\epsilon}\right)$ are solutions to the equations $d y_{t}^{\epsilon}=(1 / \sqrt{\epsilon}) \sum_{k=1}^{N} A_{k}\left(y_{t}^{\epsilon}\right) \circ d b_{t}^{k}+(1 / \epsilon) A_{0}\left(y_{t}^{\epsilon}\right)$, where $A_{k} \in \mathfrak{h}$, then $\pi\left(y_{t}^{\epsilon}\right)=\pi\left(y_{0}^{\epsilon}\right)$ for all $t$. The orbits of $\left(g_{t}^{\epsilon}\right)$ are perturbations to the orbits of the vertical motions $\left(y_{t}^{\epsilon}\right)$. The 'constant of motion' for the latter takes its value in the orbit manifold $G / H$. There will be of course extra difficulties, which is mainly due to the fact that our slow motions are 
not necessarily local functions of $\left(g_{t}^{\epsilon}\right)$, they depend not simply on $\left(g_{t}^{\epsilon}\right)$ but also on its whole trajectory.

For $\epsilon$ small, we would expect $\pi\left(g_{t}^{\epsilon}\right)$ to measure its deviation from the 'orbit' containing $g_{0}^{\epsilon}$. The inherited non-linearity from $\pi$ causes some technical problems, for example the homogeneous manifold is in general not parallelisable, so it is not easy to work directly with $x_{t}^{\epsilon}=\pi\left(g_{t}^{\epsilon}\right)$. The operator $(1 / \epsilon) \mathcal{L}_{0}+Y_{0}$ does not satisfy Hörmander's conditions, so we do not wish to work directly with $\left(g_{t}^{\epsilon}\right)$ either. To overcome these difficulties we use an $\operatorname{Ad}_{H}$-invariant decomposition of $\mathfrak{g}$, with which we construct a stochastic process $\left(u_{t}^{\epsilon}\right)$ on $G$, having the same projection as $\left(g_{t}^{\epsilon}\right)$. Since $\mathfrak{m}$ is $\operatorname{Ad}_{H}$ invariant, $G$ is a principal bundle over $M$ with structure group $H$. We lift $\left(x_{t}^{\epsilon}\right)$ to $G$ to obtain a 'horizontal' stochastic process $\left(\tilde{x}_{t}^{\epsilon}\right)$ covering $\left(x_{t}^{\epsilon}\right)$. The horizontality is with respect to the Ehresmann connection determined by $\mathfrak{m}$. Then $\left(u_{t}^{\epsilon}\right)$ is the horizontal lift process. As 'perturbations' to the random motions on the fibres, the horizontal lifts $\left(\tilde{x}_{t}^{\epsilon}\right)$ describe transverse motions across the fibres.

This consideration has a bonus: in addition to asymptotic analysis of the $x$ processes, we also obtain information on the asymptotic properties of their horizontal lifts. This is more striking if we get out of the picture of homogenisation for a moment, and consider instead the three dimensional Heisenberg group as a fibre bundle over $\mathbb{R}^{2}$. The horizontal lift of an $\mathbb{R}^{2}$-valued Brownian motion to the Heisenberg group is their stochastic Lévy area, c.f. [45]. Horizontal lifts of stochastic processes are standard tools in the study of Malliavin calculus in association with the study of the space of continuous paths over a Riemannian manifold. In Section 4 below we deduce an explicit equation for the horizontal lift in terms of the vertical component of $\left(g_{t}^{\epsilon}\right)$, making further analysis possible.

\section{Reduction, separation of slow and fast variables.}

The sub-group $H$ acts on $G$ on the right by group multiplication and we have a principal bundle structure $P(G, H, \pi)$ with base space $M$ and structure group $H$. Each fibres $\pi^{-1}(x)$ is diffeomorphic to $H$ and the kernel of $d \pi_{g}$, the differential at $g$, is $g \mathfrak{h}=\left\{T L_{g}(X): X \in \mathfrak{h}\right\}$. For $a \in G$, denote by $\bar{L}_{a}$ the left action on $M: \bar{L}_{a}(g H)=a g H$. Then $\pi \circ L_{a}=\bar{L}_{a} \circ \pi$ and $\left.(d \pi)_{a} d L_{a}\right|_{T_{1} G}=\left(d \bar{L}_{a}\right)(d \pi)_{1}$ where 1 denotes the unit element of $G$.

In this section we assume that $H$ contains no normal subgroup of $G$, which is equivalent to $G$ acts on $G / H$ effectively, i.e. an element of $G$ acting as the identity transformation on $G / H$ is the identity element of $g$. This is not a restriction on the homogeneous space. If $G$ does not act transitively on $M=G / H$, then there exists a normal subgroup $H^{0}$ of $H$ such that $G / H^{0}$ acts transitively on $G / H=\left(G / H^{0}\right) /\left(H / H^{0}\right)$. We identify $\mathfrak{m}$ with $T_{o} M$ as below:

$$
X \mapsto(d \pi)_{1}(X)=\left.\frac{d}{d t}\right|_{t=0} d \bar{L}_{\exp (t X)} O .
$$

An Ehresmann connection is a choice of a set of complements of $g \mathfrak{h}$ that is right invariant by the action of $H$. Our basic assumption is that $\mathfrak{g}=\mathfrak{h} \oplus \mathfrak{m}$ is a reductive decomposition, in which case $(g h) \mathfrak{m}=T R_{h}(g \mathfrak{m})$ and $T_{g} G=g \mathfrak{h} \oplus g \mathfrak{m}$ is an Ehresmann connection. See 
Kobayashi and Nomizu [40].

An Ehresmann connection determines and is uniquely determined by horizontal lifting maps $\mathfrak{h}_{u}: T_{g H} M \rightarrow T_{u} G$ where $u \in \pi^{-1}(g H)$. Furthermore, to every piecewise $C^{1}$ curve $c$ on $M$ and every initial value $\tilde{c}(0) \in \pi^{-1}(c(0))$, there is a unique curve $\tilde{c}$ covering $c$ with the property that $(d / d t) \tilde{c}(t) \in \tilde{c}(t) \mathfrak{m}$. See Besse [7]. We can also horizontally lift a sample continuous semi-martingale. The case of the linear frame bundle is specially well known, see Eells and Elworthy [17], Malliavin [50]. See also Arnaudon [1], Emery [21], Elworthy [18], Ikeda and Watanabe [36]. This study has been taken further in Elworthy, LeJan and $\mathrm{Li}[\mathbf{2 0}]$, in connection with horizontal lifts of intertwined diffusions. A continuous time Markov process, whose infinitesimal generator is in the form of the sum of squares of vector fields, is said to be horizontal if the vector fields are horizontal vector fields.

Let $\left\{b_{t}^{l}, w_{t}^{k}, k=1, \ldots, N_{1}, l=1, \ldots, N_{2}\right\}$ be real valued, not necessarily independent, Brownian motions. Let $\mathfrak{g}=\mathfrak{h} \oplus \mathfrak{m}$ be a reductive structure. Let $\left\{A_{i}, 1 \leq i \leq p\right\}$ be a basis of $\mathfrak{h},\left\{X_{j}, p+1 \leq j \leq n\right\}$ a basis of $\mathfrak{m}$, and $\left\{c_{k}^{i}, c_{l}^{j}\right\}$ is a family of real valued smooth functions on $G$. Let $\varpi$ be the canonical connection 1-form on the principal bundle $P(G, H, \pi)$, determined by $\varpi\left(A_{k}^{*}\right)=A_{k}$ whenever $A_{k} \in \mathfrak{h}$ and $\varpi\left(X_{j}^{*}\right)=0$ whenever $X_{j} \in \mathfrak{m}$. A left invariant vector field corresponding to a Lie algebra element is denoted by an upper script $*$ for emphasizing.

Definition 4.1. A semi-martingale $\left(\tilde{x}_{t}\right)$ in $G$ is horizontal if $\varpi\left(\circ d \tilde{x}_{t}\right)=0$. If $\left(x_{t}\right)$ is a semi-martingale on $M$, we denote by $\left(\tilde{x}_{t}\right)$ a horizontal lift.

Let $Y_{k}^{h}(g)=\sum_{i=p+1}^{n} c_{k}^{i}(g) X_{i}^{*}(g)$ and $Y_{l}^{v}(g)=\sum_{j=1}^{p} c_{l}^{j}(g) A_{j}^{*}(g)$. Denote by $\left(g_{t}, t<\right.$ $\zeta)$ the maximal solution to the following system of equations,

$$
d g_{t}=Y_{0}^{h}\left(g_{t}\right) d t+\sum_{k=1}^{N_{1}} Y_{k}^{h}\left(g_{t}\right) \circ d w_{t}^{k}+Y_{0}^{v}\left(g_{t}\right) d t+\sum_{l=1}^{N_{2}} Y_{l}^{v}\left(g_{t}\right) \circ d b_{t}^{l},
$$

with initial value $g_{0}$ and $x_{t}=\pi\left(g_{t}\right)$. For simplicity let $b_{t}^{0}=t$ and $w_{t}^{0}=t$.

In the lemma below, we split $\left(g_{t}\right)$ into its 'horizontal' and 'vertical part' and describe the horizontal lift of the projection of $\left(g_{t}\right)$ by an explicit stochastic differential equation where the role played by the vertical part is transparent.

LEMma 4.1. Let $x_{0}=\pi\left(g_{0}\right)$. Take $u_{0} \in \pi^{-1}\left(x_{0}\right)$ and define $a_{0}=u_{0}^{-1} g_{0}$. Let $\left(u_{t}, a_{t}, t<\eta\right)$ be the maximal solution to the following system of equations

$$
\begin{aligned}
d u_{t} & =\sum_{k=0}^{N_{1}} \sum_{i=p+1}^{n} c_{k}^{i}\left(u_{t} a_{t}\right)\left(\operatorname{Ad}\left(a_{t}\right) X_{i}\right)^{*}\left(u_{t}\right) \circ d w_{t}^{k} \\
d a_{t} & =\sum_{l=0}^{N_{2}} \sum_{j=1}^{p} c_{l}^{j}\left(u_{t} a_{t}\right) A_{j}^{*}\left(a_{t}\right) \circ d b_{t}^{l} .
\end{aligned}
$$

Then the following statements hold.

(1) $\left(u_{t} a_{t}, t<\eta\right)$ solves (4.1). Furthermore $\eta \leq \zeta$ where $\zeta$ is the life time of $\left(g_{t}\right)$. 
(2) $\left(u_{t}, t<\eta\right)$ is a horizontal lift of $\left(x_{t}, t<\zeta\right)$. Consequently $\zeta=\eta$ a.s.

(3) If $\left(\tilde{x}_{t}, t<\zeta\right)$ is an horizontal lift of $\left(x_{t}, t<\zeta\right)$, it is a solution of (4.2) with $u_{0}=\tilde{x}_{0}$.

Proof. (1) Define $\tilde{g}_{t}:=u_{t} a_{t}$. On $\{t<\eta\}$, we have

$$
d \tilde{g}_{t}=d R_{a_{t}} \circ d u_{t}+\left(a_{t}^{-1} \circ d a_{t}\right)^{*}\left(\tilde{g}_{t}\right) .
$$

Here $d R_{g}$ denotes the differential of the right translation $R_{g}, a_{t}^{-1}$ in the last term denotes the action of the differential, $d L_{\left(a_{t}\right)^{-1}}$, of the left multiplication. See page 66 of Kobayashi and Nomizu [40]. The stochastic differential $d$ on both the left and right hand side denotes Stratonovich integration. Then $\left(\tilde{g}_{t}, t<\eta\right)$ is a solution of $(4.1)$, which follows from the computations below.

$$
\begin{aligned}
d \tilde{g}_{t}= & \sum_{k=0}^{N_{1}} \sum_{i=p+1}^{n} c_{k}^{i}\left(u_{t} a_{t}\right) d R_{a_{t}}\left(\operatorname{Ad}\left(a_{t}\right) X_{i}\right)^{*}\left(u_{t}\right) \circ d w_{t}^{k} \\
& +\sum_{l=0}^{N_{2}} \sum_{j=1}^{p} c_{l}^{j}\left(u_{t} a_{t}\right) A_{j}^{*}\left(\tilde{g}_{t}\right) \circ d b_{t}^{l} .
\end{aligned}
$$

Since $d R_{a_{t}}\left(\operatorname{Ad}\left(a_{t}\right)\left(X_{j}\right)\right)^{*}\left(u_{t}\right)=X_{j}^{*}\left(\tilde{g}_{t}\right)$,

$$
d \tilde{g}_{t}=\sum_{k=0}^{N_{1}} \sum_{j=p+1}^{n} c_{k}^{j}\left(\tilde{g}_{t}\right)\left(X_{k}\right)^{*}\left(u_{t}\right) \circ d w_{t}^{k}+\sum_{l=0}^{N_{2}} Y_{l}^{v}\left(\tilde{g}_{t}\right) \circ d b_{t}^{l},
$$

which is equation (4.1). Since the coefficients of (4.1) are smooth, pathwise uniqueness holds. In particular $g_{t}=u_{t} a_{t}$ and the life time $\zeta$ of (4.1) must be greater or equal to $\eta$.

(2) It is clear that $a_{0} \in H$ and $\varpi\left(\circ d u_{t}\right)=0$. Let $y_{t}=\pi\left(u_{t}\right)$. Then

$$
d y_{t}=\sum_{k=0}^{N_{1}} \sum_{i=p+1}^{n} c_{k}^{i}\left(u_{t} a_{t}\right) d \bar{L}_{u_{t} a_{t}}\left(d \pi\left(X_{i}\right)\right) \circ d w_{t}^{k},
$$

following from the identity $d \pi\left(\left(\operatorname{Ad}(a) X_{i}\right)^{*}(u)\right)=d \bar{L}_{u} d \bar{L}_{a}\left(d \pi\left(X_{i}\right)\right)$. By the same reasoning $\left(x_{t}\right)$ satisfies the equation

$$
d x_{t}=\sum_{k=0}^{N_{1}} d \pi\left(Y_{k}^{h}\left(g_{t}\right)\right) \circ d w_{t}^{k} .
$$

By the definition, $d \pi\left(Y_{k}^{h}\left(g_{t}\right)\right)=\sum_{i=p+1}^{n} c_{k}^{i}\left(g_{t}\right) d \pi\left(X_{i}^{*}\left(g_{t}\right)\right)$ and $d \pi\left(X_{i}^{*}\left(g_{t}\right)\right)=T \bar{L}_{g_{t}} d \pi\left(X_{i}\right)$. Using part (1), $g_{t}=u_{t} a_{t}$, we conclude that the two equations above are the same and $\pi\left(u_{t}\right)=x_{t}$. This concludes that $\left(u_{t}\right)$ is a horizontal lift of $\left(x_{t}\right)$ up to time $\eta$.

It is well known that through each $u_{0}$ there is a unique horizontal lift $\left(\tilde{x}_{t}\right)$ and the life time of $\left(\tilde{x}_{t}\right)$ is the same as the life time of $\left(x_{t}\right)$. See Shigekawa [64] and Darling [14]. The life time of $\left(x_{t}\right)$ is $\zeta$. Let $\tilde{a}_{t}$ be the process such that $g_{t}=u_{t} \tilde{a}_{t}$ for $t<\eta$. On $\{t<\eta\}$, $\tilde{a}_{t}=a_{t}$ and $u_{t}=\tilde{x}_{t}$. If $\eta<\zeta$, as $t \rightarrow \eta$, $\lim _{t \rightarrow \eta} u_{t}$ leaves every compact set. This is impossible as it agrees with $\tilde{x}_{t}$. Similarly $\left(a_{t}\right)$ cannot explode before $\zeta$. 
(3) Let $\left(g_{t}, t<\zeta\right)$ be a solution of (4.1) and set $x_{t}=\pi\left(g_{t}\right)$. For each $t, g_{t}$ and $\tilde{x}_{t}$ belong to the same fibre. Define $k_{t}=\tilde{x}_{t}^{-1} g_{t}$, which takes values in $H$ and is defined for all $t<\zeta$. Then,

$$
d \tilde{x}_{t}=d R_{\left(k_{t}\right)^{-1}} \circ d g_{t}+\left(k_{t} \circ d\left(k_{t}\right)^{-1}\right)^{*}\left(\tilde{x}_{t}\right) .
$$

From this and equation (4.1) we obtain the following,

$$
\begin{aligned}
d \tilde{x}_{t}= & d R_{\left(k_{t}\right)^{-1}}\left(\sum_{k=0}^{N_{1}} \sum_{i=p+1}^{n} c_{k}^{i}\left(g_{t}\right) X_{i}^{*}\left(g_{t}\right) \circ d w_{t}^{k}\right) \\
& +d R_{\left(k_{t}\right)^{-1}}\left(\sum_{l=0}^{N_{2}} \sum_{j=1}^{p} c_{l}^{j}\left(g_{t}\right) A_{j}^{*}\left(g_{t}\right) \circ d b_{t}^{l}\right)+\left(\left(k_{t}\right) \circ d\left(k_{t}\right)^{-1}\right)^{*}\left(\tilde{x}_{t}\right) .
\end{aligned}
$$

We apply the connection 1-form $\varpi$ to equation (4.4), observing $\omega\left(\circ d \tilde{x}_{t}\right)=0$ and $\operatorname{Ad}\left(k_{t}\right)\left(X_{i}\right) \in \mathfrak{m}$

$$
\begin{aligned}
0 & =\sum_{l=0}^{N_{2}} \sum_{j=1}^{p} c_{l}^{j}\left(g_{t}\right) \varpi_{\tilde{x}_{t}}\left(d R_{\left(k_{t}\right)^{-1}} A_{j}^{*}\left(g_{t}\right)\right) \circ d b_{t}^{l}+\left(k_{t}\right) \circ d\left(k_{t}\right)^{-1} \\
& =\sum_{l=0}^{N_{2}} \sum_{j=1}^{p} c_{l}^{j}\left(g_{t}\right) \operatorname{Ad}\left(k_{t}\right) A_{j} \circ d b_{t}^{l}+\left(k_{t}\right) \circ d\left(k_{t}\right)^{-1}
\end{aligned}
$$

We have used the fact that $X_{j}^{*}$ are horizontal, $d R_{\left(k_{t}\right)^{-1}}\left(\left(X_{j}\right)^{*}\left(g_{t}\right)\right)=\left(\operatorname{Ad}\left(k_{t}\right)\left(X_{j}\right)\right)^{*}\left(\tilde{x}_{t}\right)$, and $\varpi_{g a^{-1}}\left(R_{\left(a^{-1}\right)_{*}} w\right)=\operatorname{Ad}(a) \varpi_{g}(w)$ for any $w \in T_{g} G$. It follows that $d\left(k_{t}\right)^{-1}=$ $-\sum_{l=0}^{N_{2}} \sum_{j=1}^{p} c_{l}^{j}\left(g_{t}\right) R_{\left(k_{t}\right)^{-1}} A_{j} \circ d b_{t}^{l}$. By the product rule,

$$
d k_{t}=\sum_{l=0}^{N_{2}} \sum_{j=1}^{p} c_{l}^{j}\left(g_{t}\right) A_{j}^{*}\left(k_{t}\right) \circ d b_{t}^{l} .
$$

Thus $\left(k_{t}\right)$ solves equation (4.3) and we take this back to (4.4). Since the vertical vector field associated to $k_{t} \circ d k_{t}^{-1}$ evaluated at $\tilde{x}_{t}$ is given by the formula

$$
\left(k_{t} \circ d\left(k_{t}\right)^{-1}\right)^{*}\left(\tilde{x}_{t}\right)=-\sum_{l=0}^{N_{2}} \sum_{j=1}^{p} c_{l}^{j}\left(g_{t}\right)\left(\operatorname{Ad}\left(k_{t}\right) A_{j}\right)^{*}\left(\tilde{x}_{t}\right) \circ d b_{t}^{l},
$$

the second term and the third term on the right hand side of (4.4) cancel. Using the same computation given earlier, we see that

$$
\begin{aligned}
d \tilde{x}_{t} & =d R_{\left(k_{t}\right)^{-1}}\left(\sum_{k=0}^{N_{1}} \sum_{i=p+1}^{n} c_{k}^{i}\left(g_{t}\right) X_{i}^{*}\left(g_{t}\right) \circ d w_{t}^{k}\right) \\
& =\sum_{k=0}^{N_{1}} \sum_{i=p+1}^{n} c_{k}^{i}\left(\tilde{x}_{t} k_{t}\right)\left(\operatorname{Ad}\left(k_{t}\right) X_{i}\right)^{*}\left(\tilde{x}_{t}\right) \circ d w_{t}^{k},
\end{aligned}
$$


proving that $\left(\tilde{x}_{t}, t<\zeta\right)$ is a solution of (4.2) and concludes the proof. In particular $\zeta \geq \tau$.

We observe that the Ehresmann connection induced by the reductive decomposition is independent of the scaling of the Riemannian metric.

Corollary 4.2. Let $\epsilon_{l}>0$ and $Y_{0} \in \mathfrak{m}$. Let $\left(g_{t}^{\epsilon}\right)$ be a solution to the equation

$$
d g_{t}^{\epsilon}=Y_{0}^{h}\left(g_{t}^{\epsilon}\right) d t+\sum_{k=1}^{N_{1}} Y_{k}^{h}\left(g_{t}^{\epsilon}\right) \circ d w_{t}^{k}+\frac{1}{\epsilon_{0}} Y_{0}^{v}\left(g_{t}^{\epsilon}\right) d t+\sum_{l=1}^{N_{2}} \frac{1}{\epsilon_{l}} Y_{k}^{v}\left(g_{t}^{\epsilon}\right) \circ d b_{t}^{l}, \quad g_{0}^{\epsilon}=g_{0} .
$$

Then the horizontal lift of $\pi\left(g_{t}^{\epsilon}\right)$ satisfies the following system of equations

$$
\begin{aligned}
d \tilde{x}_{t}^{\epsilon}= & \sum_{k=1}^{N_{1}} \sum_{i=p+1}^{n} c_{k}^{i}\left(\tilde{x}_{t}^{\epsilon} a_{t}^{\epsilon}\right)\left(\operatorname{Ad}\left(a_{t}^{\epsilon}\right) X_{i}\right)^{*}\left(\tilde{x}_{t}^{\epsilon}\right) \circ d w_{t}^{k} \\
& +\sum_{i=p+1}^{n} c_{0}^{i}\left(\tilde{x}_{t}^{\epsilon} a_{t}^{\epsilon}\right)\left(\operatorname{Ad}\left(a_{t}^{\epsilon}\right) X_{i}\right)^{*}\left(\tilde{x}_{t}^{\epsilon}\right) d t, \\
d a_{t}^{\epsilon}= & \sum_{l=1}^{N_{2}} \frac{1}{\epsilon_{l}} \sum_{j=1}^{p} c_{l}^{j}\left(\tilde{x}_{t}^{\epsilon} a_{t}^{\epsilon}\right) A_{j}^{*}\left(a_{t}^{\epsilon}\right) \circ d b_{t}^{l}+\sum_{j=1}^{p} \frac{1}{\epsilon_{0}} c_{0}^{j}\left(\tilde{x}_{t}^{\epsilon} a_{t}^{\epsilon}\right) A_{j}^{*}\left(a_{t}^{\epsilon}\right) d t,
\end{aligned}
$$

up to an explosion time. Here $\tilde{x}_{0}^{\epsilon}=g_{0}$ and $a_{0}^{\epsilon}$ is the identity.

EXAmple 4.3. Let us take the Hopf fibration $\pi: S U(2) \rightarrow S^{2}(1 / 2)$, given the bi-invariant metric. If we represent $S U(2)$ by the unit sphere in $\mathbb{C}^{2}$ and $S^{2}(1 / 2)$ as a subset in $\mathbb{R} \oplus \mathbb{C}$, the Hopf map is given by the formula $\pi(z, w)=\left(\left(|w|^{2}-|z|^{2}\right) / 2, z \bar{w}\right)$. It is a Riemannian submersion. Let $\left\{X_{1}, X_{2}, X_{3}\right\}$ be Pauli matrices defined by (2.1) and let $\mathfrak{m}=\left\langle X_{2}, X_{3}\right\rangle$. Then $[\mathfrak{m}, \mathfrak{h}] \subset \mathfrak{m}$. This is easily seen from the structure of the Lie bracket: $\left[X_{1}, X_{2}\right]=-2 X_{3},\left[X_{2}, X_{3}\right]=-2 X_{1},\left[X_{3}, X_{1}\right]=-2 X_{2}$. Let $\left(g_{t}^{\epsilon}\right)$ be a solution to the equation (2.2) and $x_{t}^{\epsilon}=\pi\left(g_{t}^{\epsilon}\right)$. Denote by $\left(u_{t}^{\epsilon}\right)$ the horizontal lift of $\left(x_{t}^{\epsilon}\right)$. By Corollary $4.2,\left(u_{t}^{\epsilon}, a_{t}^{\epsilon}\right)$ satisfies

$$
\begin{aligned}
& d u_{t}^{\epsilon}=\left(\operatorname{Ad}\left(a_{t}^{\epsilon}\right) X_{2}\right)\left(u_{t}^{\epsilon}\right) \circ d b_{t}^{2}+\left(\operatorname{Ad}\left(a_{t}^{\epsilon}\right) X_{3}\right)\left(u_{t}^{\epsilon}\right) \circ d b_{t}^{3}, \\
& d a_{t}^{\epsilon}=\frac{1}{\sqrt{\epsilon}} X_{1}\left(a_{t}^{\epsilon}\right) \circ d b_{t}^{1} .
\end{aligned}
$$

Since the metric is invariant by $\operatorname{Ad}_{H},\left(u_{t}^{\epsilon}, t \geq 0\right)$ is a horizontal Brownian motion, its Markov generator is the horizontal Laplacian $\Delta^{h}=\operatorname{trace} \nabla_{\mathfrak{m}} d$. Furthermore $\left\{\pi_{*}\left(\left(\operatorname{Ad}\left(a_{t}^{\epsilon}\right) X_{2}\right)^{*}\right), \pi_{*}\left(\left(\operatorname{Ad}\left(a_{t}^{\epsilon}\right) X_{3}\right)^{*}\right)\right\}$ is an orthonormal frame in $S^{2}(1 / 2)$ and then for each $\epsilon>0,\left(x_{t}^{\epsilon}, t \geq 0\right)$ is a Brownian motion on $S^{2}(1 / 2)$.

Corollary 4.4. Let $Y_{0} \in \mathfrak{m}, A_{0} \in \mathfrak{h}$ and $\left\{A_{k}, 1 \leq k \leq N\right\} \subset \mathfrak{h}$. If there is an $\operatorname{Ad}_{H}$ invariant inner product on $\mathfrak{g}$, the following SDEs are conservative for every $\epsilon$.

$$
\dot{u}_{t}^{\epsilon}=\left(\operatorname{Ad}\left(h_{t / \epsilon}\right) Y_{0}\right)^{*}\left(u_{t}^{\epsilon}\right),
$$




$$
\begin{aligned}
& d h_{t}=\sum_{k=1}^{N} A_{k}^{*}\left(h_{t}\right) \circ d b_{t}^{k}+A_{0}^{*}\left(h_{t}\right) d t, \\
& d g_{t}^{\epsilon}=Y_{0}^{*}\left(g_{t}^{\epsilon}\right) d t+\frac{1}{\sqrt{\epsilon}} \sum_{k=1}^{N} A_{k}^{*}\left(g_{t}^{\epsilon}\right) \circ d b_{t}^{k}+\frac{1}{\epsilon} A_{0}^{*}\left(g_{t}^{\epsilon}\right) d t .
\end{aligned}
$$

Furthermore $\pi\left(g_{t}^{\epsilon}\right)=\pi\left(u_{t}^{\epsilon}\right)$, and $\left(g_{t}^{\epsilon}\right)$ and $\left(u_{t}^{\epsilon} h_{t / \epsilon}\right)$ are equal in law.

Proof. With respect to the left invariant Riemannian metric on $G$ generated by the $\operatorname{Ad}_{H}$-invariant inner product, the random vector fields $\left(\operatorname{Ad}\left(h_{t / \epsilon}^{\epsilon}\right) Y_{0}\right)^{*}$ are bounded, so (4.7) is conservative for almost surely all $\omega$. That (4.8) does not explode is clear, c.f. Lemma 5.1 below. Since $\pi\left(g_{t}^{\epsilon}\right)=\pi\left(u_{t}^{\epsilon}\right)$, by Lemma $4.1,(4.9)$ is also conservative.

REMARK. By the averaging principle we expect that the processes $\left\{u_{.}^{\epsilon}, \epsilon>0\right\}$ converge to the solution of the ODE

$$
\dot{u}_{t}=\int_{H}\left(\operatorname{Ad}(h)\left(Y_{0}\right)\right)^{*}\left(u_{t}\right) d h
$$

where $d h$ is the Haar measure on $H$. This can be seen, assuming $G$ compact for simplicity, by sub-dividing $[0, t / \epsilon]$ into sub-intervals of size $\epsilon^{-\delta}$ for a suitable positive number $\delta$ and by the law of large numbers for Brownian motions on a compact manifold, stochastic averaging of stochastic slow-fast systems is treated in [48]. Our main aim is to study the effective diffusion on the next time scale in case $\bar{Y}_{0}:=\int_{H} \operatorname{Ad}(h)(Y) d h$ vanishes. We do not need an explicit statement on the averaging principle, and will therefore refer the interested reader to $[\mathbf{4 5}]$ and also to $[\mathbf{4 4}]$.

\section{Elementary lemmas.}

Let $\left\{X_{k}, k=1, \ldots, m\right\}$ be smooth vector fields on a smooth manifold $N$. Denote by $\mathcal{L}$ the Hörmander type operator $(1 / 2) \sum_{k=1}^{m}\left(X_{k}\right)^{2}+X_{0}$. If at each point, $X_{1}, \ldots$, $X_{m}$ and their Lie brackets generate the tangent space, we say that $\mathcal{L}$ satisfies strong Hörmander's condition. It satisfies Hörmander's condition if $X_{0}$ is allowed. A Hörmander type operator on a compact manifold satisfying strong Hörmander's condition has a unique invariant probability measure $\pi$; furthermore for $f \in C^{\infty}(N ; \mathbb{R}), \mathcal{L} F=f$ is solvable if and only if $\int f d \pi=0$. We denote by $\mathcal{L}^{-1} f$ a solution to the Poisson equation $\mathcal{L} F=f$ whenever it exists. If a Markov operator $\mathcal{L}$ has a unique invariant probability measure $\pi$ and $f \in L^{1}(N ; \pi)$ we write $\bar{f}=\int_{N} f d \pi$.

Lemma 5.1. Let $G$ be a Lie group with left invariant Riemannian metric. Then an SDE driven by left invariant vector fields is conservative. If $\mu$ is a right invariant measure on $G$ then left invariant vector fields are divergence free and the linear operator $\mathcal{B}=(1 / 2) \sum_{i=1}^{m}\left(X_{i}\right)^{2}$, where $X_{i} \in \mathfrak{g}$, is symmetric on $L^{2}(G ; d \mu)$.

PROOF. The SDE is conservative follows from the fact that a Lie group with left invariant metric is geodesically complete and has positive injectivity radius. The left invariant vector fields and their covariant derivatives are bounded, so by localisa- 
tion or the uniform cover criterion in Elworthy [18, Chapter vii], solutions of equation (4.8) from any initial point exist for all time. If $f \in B C^{1}(G ; \mathbb{R})$, using the right invariance of the measure $\mu, \int_{G}(X f)(g) \mu(d g)=\left.\int_{G}(d / d t) f(g \exp (t X))\right|_{t=0} \mu(d g)=0$. Consequently $X$ having vanishing divergence with respect to $\mu$ and for $f_{1}, f_{2} \in B C^{1}(G ; \mathbb{R})$, $\int_{G} f_{2}\left(X f_{1}\right) d \mu=-\int_{G} f_{1}\left(X f_{2}\right) d \mu$. In particular $\mathcal{B}$ is symmetric on $L^{2}(G, \mu)$.

We say a family of vectors $\left\{A_{1}, \ldots, A_{N}\right\}$ in $\mathfrak{h} \subset \mathfrak{g}$ is Lie algebra generating if $\left\{A_{1}, \ldots, A_{N}\right\}$ and their iterated brackets generate $\mathfrak{h}$. Define $\mathcal{L}_{0}=(1 / 2) \sum_{k=1}^{N}\left(A_{k}\right)^{2}+A_{0}$. We restrict $A_{k}$ to the compact manifold $H$ and treat $\mathcal{L}_{0}$ as an operator on $H$. If $\mathcal{L}_{0}$ is symmetric and satisfies Hörmander's condition, the maximal principle states that $\mathcal{L}_{0}^{*} u=0$ has only constant solutions.

Lemma 5.2. If $H$ is compact and $\left\{A_{0}, A_{1}, \ldots, A_{N}\right\} \subset \mathfrak{h}$ is Lie algebra generating, the following statements hold.

1. The normalised Haar measure $d h$ is the unique invariant probability measure for $\mathcal{L}_{0}$, and $\mathcal{L}_{0}$ is a Fredholm operator with Fredholm index 0.

2. If $\int_{H}\left\langle\operatorname{Ad}(h)\left(Y_{0}\right), Y\right\rangle d h=0$, where $Y \in \mathfrak{g}$, there is a unique function $F \in C^{\infty}(H ; \mathbb{R})$ solving the Poisson equation $\mathcal{L}_{0} F=\left\langle\operatorname{Ad}(\cdot)\left(Y_{0}\right), Y\right\rangle$.

Proof. Denote by $\mathcal{L}_{0}^{*}$ the dual of $\mathcal{L}_{0}$ on $L^{2}(H ; \mathbb{R})$ which, by Lemma 5.1 , is $\mathcal{L}_{0}^{*}=\sum_{k}\left(A_{k}\right)^{2}-A_{0}$. Both $\mathcal{L}_{0}$ and $\mathcal{L}_{0}^{*}$ satisfies Hörmander's condition. We have seen that $\int_{H} \mathcal{L}_{0} f d h$ vanishes for all $f \in C^{\infty}$ and $d h$ is an invariant measure, with full topological support. Distinct ergodic invariant measures have disjoint supports, and since every invariant measure is a convex combination of ergodic invariant measures, the Haar measure is therefore the only invariant measure, up to a scaling. Also $\mathcal{L}_{0}$ satisfies a sub-elliptic estimate: $\|u\|_{s} \leq\left\|\mathcal{L}_{0} u\right\|_{L^{2}}+c\|u\|_{L^{2}}$, Hörmander [33], which implies that $\mathcal{L}_{0}$ has compact resolvent, and $\mathcal{L}_{0}$ is a Fredholm operator. In particular $\mathcal{L}_{0}$ has closed range, Hörmander [34] and $\mathcal{L}_{0} u=\left\langle\operatorname{Ad}(h)\left(Y_{0}\right), Y\right\rangle$ is solvable if and only if $\left\langle\operatorname{Ad}(h)\left(Y_{0}\right), Y\right\rangle$ annihilates the kernel of $\mathcal{L}_{0}^{*}$, i.e. $\int_{H}\left\langle\operatorname{Ad}(h)\left(Y_{0}\right), Y\right\rangle d h$ vanishes. By the earlier argument the dimension of the kernels of $\mathcal{L}_{0}$ and $\mathcal{L}_{0}^{*}$ agree and $\mathcal{L}_{0}$ has Fredholm index 0.

\section{Diffusion creation and rate of convergence.}

Let $\left(h_{t}\right)$ be a Markov process on a compact manifold $H$ with generator $\mathcal{L}_{0}=$ $\sum_{k}\left(A_{k}\right)^{2}+A_{0}$ where $A_{k}$ are smooth vector fields satisfying Hörmander's condition, and a invariant probability measure $\mu$. Let $\Phi_{t}^{\epsilon}(y)$ be the solution to a family of conservative random differential equations $\dot{y}_{t}^{\epsilon}=\sum_{k=1}^{m} \alpha_{k}\left(h_{t / \epsilon}\right) Y_{k}\left(y_{t}^{\epsilon}\right)$ with $y_{0}^{\epsilon}=y_{0}$ and $Y_{k}$ smooth vector fields.

Lemma 6.1. Suppose that $N$ is compact; or satisfies the following conditions.

- The injectivity radius of $N$ is greater than a positive number $2 a$.

- For a Riemannian distance function $\rho$ on $N$,

$$
C_{1}(p):=\sup _{s, t \leq 1} \sup _{x \in N} \boldsymbol{E}\left(\left|Y_{j}\left(y_{t / \epsilon}^{\epsilon}\right)\right|^{p} \mathbf{1}_{\rho\left(y_{t / \epsilon}^{\epsilon}, x\right) \leq 2 a}\right)<\infty,
$$


for all $p ;$ also $C_{2}(p):=\sup _{s, t \leq 1} \sup _{x \in N} \boldsymbol{E}\left(\left|\nabla d \rho^{2}\left(y_{t / \epsilon}^{\epsilon}, x\right)\right|^{p} \mathbf{1}_{\rho\left(y_{t / \epsilon}^{\epsilon}, x\right) \leq 2 a}\right)$ is finite.

Then $\left(y_{t / \epsilon}^{\epsilon}\right)$ converge weakly to a Markov process with generator $-\sum_{i, j} \overline{\alpha_{i} \mathcal{L}_{0}^{-1} \alpha_{j}} L_{Y_{i}} L_{Y_{j}}$.

Proof. Let $\beta_{j}=\mathcal{L}_{0}^{-1} \alpha_{j}$ and we first prove the tightness of the family of stochastic processes $\left\{y_{t / \epsilon}^{\epsilon}, \epsilon>0\right\}$. Note that

$$
\rho\left(y_{s / \epsilon}^{\epsilon}, y_{t / \epsilon}^{\epsilon}\right)=\int_{s / \epsilon}^{t / \epsilon} \nabla \rho\left(y_{s / \epsilon}^{\epsilon}, y_{r}^{\epsilon}\right)\left(\sum_{k=1}^{m} \alpha_{k}\left(h_{r / \epsilon}\right) Y_{k}\left(y_{r}^{\epsilon}\right)\right) d r,
$$

where the gradient is on the second variable. At the first glance we expect that $\rho\left(y_{t / \epsilon}^{\epsilon}, y_{s / \epsilon}^{\epsilon}\right)$ to be of order $(t-s) / \epsilon$, we will use a trick, the Itô trick, to overcome this problem, see $[\mathbf{4 4}]$ where it was also used. We may assume that $\left(h_{t}\right)$ solves (4.8). Since $\mathcal{L}_{0} \beta_{j}=\alpha_{j}$,

$$
\beta_{j}\left(h_{t / \epsilon}\right)=\beta_{j}\left(h_{0}\right)+\int_{0}^{t / \epsilon} \alpha_{j}\left(h_{r}\right) d r+M_{t / \epsilon}^{\epsilon}
$$

where $M_{t / \epsilon}^{\epsilon}$ denotes a local martingale, and $\int_{0}^{t / \epsilon} \alpha_{j}\left(h_{r}\right) d r$ is of order 1 , modulus the fast oscillating local martingale. To use this in our setting let us take a $C^{2}$ function $f: G \rightarrow \mathbb{R}$, by the product rule:

$$
\begin{aligned}
d f\left(Y_{j}\left(y_{t / \epsilon}^{\epsilon}\right)\right) \beta_{j}\left(h_{t / \epsilon^{2}}\right)= & d f\left(Y_{j}\left(y_{s / \epsilon}^{\epsilon}\right)\right) \beta_{j}\left(h_{s / \epsilon^{2}}\right)+\sum_{j=1}^{m} \int_{s / \epsilon}^{t / \epsilon} L_{Y_{i}} L_{Y_{j}} f\left(y_{r}^{\epsilon}\right) \alpha_{i}\left(h_{r / \epsilon}\right) \beta_{j}\left(h_{r / \epsilon}\right) d r \\
& +\frac{1}{\sqrt{\epsilon}} \sum_{k=1}^{m^{\prime}} \int_{s / \epsilon}^{t / \epsilon} L_{Y_{j}} f\left(y_{r}^{\epsilon}\right) d \beta_{j}\left(A_{k}\left(h_{r / \epsilon}\right)\right) d b_{r}^{k} \\
& +\frac{1}{\epsilon} \int_{s / \epsilon}^{t / \epsilon} L_{Y_{j}} f\left(y_{r}^{\epsilon}\right) \mathcal{L}_{0} \beta_{j}\left(h_{r / \epsilon}\right) d r .
\end{aligned}
$$

Since the Riemannian distance function fails to be $C^{2}$ if the two points are on the cut locus of each other, we take a smooth function $\phi: \mathbb{R}_{+} \rightarrow \mathbb{R}_{+}$such that $\phi(r)=r$ if $r<a$ and $\phi(r)=1$ for all $r>2 a$ and define $\tilde{\rho}=\phi \circ \rho$. We inverse engineer with the last term in the equation above, replacing $f$ by $\tilde{\rho}^{2}$ to see that, c.f. [47, Lemma 3.1], for $s, t \leq 1$,

$$
\begin{aligned}
\tilde{\rho}^{2}\left(y_{s / \epsilon}^{\epsilon}, y_{t / \epsilon}^{\epsilon}\right)= & \int_{s / \epsilon}^{t / \epsilon} \sum_{k=1}^{m} L_{Y_{k}\left(y_{r}^{\epsilon}\right)}\left(\tilde{\rho}^{2}\right)\left(y_{s / \epsilon}^{\epsilon}, y_{r}^{\epsilon}\right)\left(\alpha_{k}\left(h_{r / \epsilon}\right)\right) d r \\
= & \epsilon \sum_{j=1}^{m}\left(L_{Y_{j}} \tilde{\rho}^{2}\left(y_{s / \epsilon}^{\epsilon}, y_{t / \epsilon}^{\epsilon}\right)\right) \beta_{j}\left(h_{t / \epsilon^{2}}\right) \\
& -\epsilon \sum_{i, j=1}^{m} \int_{s / \epsilon}^{t / \epsilon}\left(L_{Y_{i}} L_{Y_{j}} \tilde{\rho}^{2}\left(y_{s / \epsilon}^{\epsilon}, y_{r}^{\epsilon}\right)\right) \alpha_{i}\left(h_{r / \epsilon}\right) \beta_{j}\left(h_{r / \epsilon}\right) d r \\
& -\sqrt{\epsilon} \sum_{j=1}^{m} \sum_{k=1}^{m^{\prime}} \int_{s / \epsilon}^{t / \epsilon}\left(L_{Y_{j}} \tilde{\rho}^{2}\right)\left(y_{s / \epsilon}^{\epsilon}, y_{r}^{\epsilon}\right)\left(L_{A_{k}} \beta_{j}\right)\left(h_{r / \epsilon}\right) d b_{r}^{k} .
\end{aligned}
$$


We raise both sides to the power $p$ where $p>2$ to see for a constant $c_{p}$ depending on $\left|\beta_{j}\right|_{\infty},\left|\alpha_{j}\right|_{\infty},\left|A_{k}\right|_{\infty}, m$, and $p, C_{1}$ and $C_{2}$, which may represent a different number in a different line,

$$
\begin{aligned}
& \boldsymbol{E}\left[\tilde{\rho}^{2 p}\left(y_{s / \epsilon}^{\epsilon}, y_{t / \epsilon}^{\epsilon}\right)\right] \\
\leq & c_{p} \epsilon^{p} \sum_{j=1}^{m} \boldsymbol{E}\left|L_{Y_{j}} \tilde{\rho}^{2}\left(y_{s / \epsilon}^{\epsilon}, y_{t / \epsilon}^{\epsilon}\right)\right|^{p}+c_{p} \epsilon^{p} \sum_{i, j=1}^{m} \boldsymbol{E}\left(\int_{s / \epsilon}^{t / \epsilon}\left|L_{Y_{i}} L_{Y_{j}} \tilde{\rho}^{2}\left(y_{s / \epsilon}^{\epsilon}, y_{r}^{\epsilon}\right)\right| d r\right)^{p} \\
& +c_{p} \epsilon^{p / 2} \sum_{j=1}^{m} \sum_{k=1}^{m^{\prime}} \boldsymbol{E}\left(\int_{s / \epsilon}^{t / \epsilon}\left|L_{Y_{j}} \tilde{\rho}^{2}\left(y_{s / \epsilon}^{\epsilon}, y_{r}^{\epsilon}\right)\right|^{2} d r\right)^{p / 2} \\
\leq & c_{p} C_{1}(p) \epsilon^{p}+c_{p}(t-s)^{p} \sqrt{C_{1}(4 p)} \sqrt{C_{2}(2 p)}+c_{p}(t-s)^{p / 2} \sqrt{C_{1}(2 p)} \sqrt{C_{2}(2 p)} .
\end{aligned}
$$

Applying $\tilde{\rho}$ directly to $y_{t / \epsilon}^{\epsilon}$ giving another estimate:

$$
\begin{aligned}
\boldsymbol{E}\left|\tilde{\rho}^{2}\left(y_{s / \epsilon}^{\epsilon}, y_{t / \epsilon}^{\epsilon}\right)\right|^{p} & \leq c_{p} \boldsymbol{E} \sum_{j}\left|\int_{s / \epsilon}^{t / \epsilon} L_{Y_{j}} \tilde{\rho}^{2}\left(y_{s / \epsilon}^{\epsilon}, y_{r / \epsilon}^{\epsilon}\right) \alpha_{j}\left(h_{r / \epsilon}\right) d r\right|^{p} \\
& \leq c_{p}\left(\frac{t-s}{\epsilon}\right)^{p} \sqrt{C_{1}(2 p)} \sqrt{C_{2}(2 p)} .
\end{aligned}
$$

Interpolate the estimates for $\epsilon^{p} \leq(t-s)^{p / 2}$ and for $\epsilon^{p} \geq(t-s)^{p / 2}$, to see

$$
\boldsymbol{E}\left|\tilde{\rho}\left(y_{s / \epsilon}^{\epsilon}, y_{t / \epsilon}^{\epsilon}\right)\right|^{2 p} \leq c_{p}|t-s|^{p / 2} .
$$

Taking $p>4$ and applying Kolmogorov's theorem we obtain the required tightness. The weak convergence follows just as for the proof of [47, Theorem 5.4], using a law of large numbers with rate of convergence the square root of time [47, Lemma 5.2]. For a Fredholm operator $\mathcal{L}_{0}$ of index zero, the limit is identified as following. Let $\left\{u_{i}, i=\right.$ $\left.1, \ldots, n_{0}\right\}$ be a basis of $\operatorname{ker}\left(\mathcal{L}_{0}\right)$ and $\left\{\pi_{i}, i=1, \ldots, n_{0}\right\}$ the dual basis for the null space of $\mathcal{L}_{0}^{*}$. Then $\overline{\mathcal{L}}=-\sum_{i, j} \sum_{b=1}^{n_{0}} u_{b}\left\langle\alpha_{i} \beta_{j}, \pi_{b}\right\rangle L_{Y_{i}} L_{Y_{j}}$, where the bracket denotes the dual pairing between $L^{2}$ and $\left(L^{2}\right)^{*}$. In our case there is only one invariant probability measure for $\mathcal{L}_{0}$, from which we conclude that $\overline{\mathcal{L}}=-\sum_{i, j} \overline{\alpha_{i} \beta_{j}} L_{Y_{i}} L_{Y_{j}}$.

Let use return to our equations on the product space $G \times H$,

$$
\begin{aligned}
\dot{u}_{t}^{\epsilon} & =\left(\operatorname{Ad}\left(h_{t / \epsilon}\right) Y_{0}\right)^{*}\left(u_{t}^{\epsilon}\right), \quad u_{0}^{\epsilon}=u_{0} \\
d h_{t} & =\sum_{k=1}^{N} A_{k}^{*}\left(h_{t}\right) \circ d b_{t}^{k}+A_{0}^{*}\left(h_{t}\right) d t, \quad \mathfrak{h}_{0}=1 .
\end{aligned}
$$

Let $x_{\cdot / \epsilon}^{\epsilon}=\pi\left(u_{\cdot / \epsilon}^{\epsilon}\right)$ and $x_{0}=\pi\left(u_{0}\right)$. Let $\langle$,$\rangle denote a left invariant and \operatorname{Ad}_{H}$ invariant scalar product on $\mathfrak{g},\left\{Y_{j}\right\}$ an orthonormal basis of $\mathfrak{m}$ and define

$$
\alpha\left(Y_{0}, Y_{j}\right)(h)=\left\langle\operatorname{Ad}(h)\left(Y_{0}\right), Y_{j}\right\rangle .
$$


Also denote by $d h$ the normalised Haar measure on $H$, we define

$$
\bar{Y}_{0} \equiv \int_{H} \operatorname{Ad}(h)\left(Y_{0}\right) d h .
$$

Proposition 6.2. Suppose that the subgroup $H$ is compact, $\left\{A_{0}, A_{1}, \ldots, A_{N}\right\}$ is a Lie algebra-generating subset of $\mathfrak{h}$, and $Y_{0} \in \mathfrak{m}$ is such that $\bar{Y}_{0}=0$. Let $T$ be a positive number. Then as $\epsilon \rightarrow 0,\left(u_{s / \epsilon}^{\epsilon}, s \leq T\right)$ converge weakly to a Markov process $\left(\bar{u}_{s}, s \leq T\right)$, whose Markov generator is

$$
\overline{\mathcal{L}}=-\sum_{i, j=1}^{m} \overline{\alpha\left(Y_{0}, Y_{i}\right)\left(\mathcal{L}_{0}^{-1} \alpha\left(Y_{0}, Y_{j}\right)\right)} L_{Y_{i}^{*}} L_{Y_{j}^{*}}
$$

Also, $\left(x_{s / \epsilon}^{\epsilon}, s \leq T\right)$ converges weakly to a stochastic process $\left(\bar{x}_{s}, s \leq T\right)$.

Proof. By the left invariance of the Riemannian metric, Equation (6.1) is equivalent to $u_{t}^{\epsilon}=\sum_{j=1}^{N} \alpha\left(Y_{0}, Y_{j}\right)\left(h_{t / \epsilon}\right) Y_{j}^{*}\left(u_{t}^{\epsilon}\right)$ and

$$
\left(\operatorname{Ad}(h)\left(Y_{0}\right)\right)^{*}(g)=\sum_{j=1}^{N}\left\langle\left(\operatorname{Ad}(h)\left(Y_{0}\right)\right)^{*}, Y_{j}^{*}\right\rangle Y_{j}^{*}(g)=\sum_{j=1}^{N} \alpha\left(Y_{0}, Y_{j}\right)(h) Y_{j}^{*}(g) .
$$

We may rewrite $(6.1)$ as $\dot{u}_{t}^{\epsilon}=\sum_{j=1}^{N} Y_{j}\left(u_{t}^{\epsilon}\right) \alpha\left(Y_{0}, Y_{j}\right)\left(h_{t / \epsilon}\right)$. Since $\bar{Y}_{0}$ vanishes apply Lemma 5.2 , so $\mathcal{L}_{0}^{-1} \alpha_{j}$ exists and is smooth for each $j$. Furthermore by Lemma 5.1, the $\overline{\mathcal{L}}$ diffusions exist for all time. Since the Riemannian metric on $G$ is left invariant, its Riemannian distance function $\rho$ is also left invariant, $\rho\left(g g_{1}, g g_{2}\right)=\rho\left(g_{1}, g_{2}\right)$ for any $g, g_{1}, g_{2} \in G$. Furthermore, $\sup _{p: \rho(p, y)<\delta} \nabla^{2} \rho(y, p)$ is finite, for sufficiently small $\delta>0$, and is independent of $y$, where $\nabla$ is the Levi-Civita connection. Thus $C_{1}(p)$ and $C_{2}(p)$, from Lemma 6.1 , are both finite. We observe that $G$ has positive injectivity radius and bounded geometry, and we then apply Lemma 6.1 to conclude that $\left(u_{s / \epsilon}^{\epsilon}, s \leq t\right)$ converges weakly as $\epsilon$ approaches 0 . As any continuous real valued function $f$ on $M$ lifts to a continuous function on $G$, the weak convergence passes, trivially, to the processes $\left(x_{t / \epsilon}^{\epsilon}\right)$.

For $p \geq 1$, denote by $W_{p}$ or $W_{p}(N)$ the Wasserstein distance on probability measures over a metric space $N$. For $T$ a positive number, let $C_{x}([0, T] ; N)$ denote the space of continuous curves defined on the interval $[0, T]$ starting from $x$. Given two probability measures $\mu_{1}, \mu_{2}$ and $C_{x}([0, T] ; N)$,

$$
W_{p}\left(\mu_{1}, \mu_{2}\right):=\left(\inf \int \sup _{0 \leq s \leq T} \rho^{p}\left(\sigma_{1}(s), \sigma_{2}(s)\right) d \nu\left(\sigma_{1}, \sigma_{2}\right)\right)^{1 / p} .
$$

Here $\sigma_{1}$ and $\sigma_{2}$ take values in $C_{x}([0, T] ; N)$ and the infimum is taken over all probability measures $\nu$ whose marginals are $\mu_{1}, \mu_{2}$.

REMARK 6.3. The weak convergence of a sequence of stochastic processes $\left\{y_{s / \epsilon}^{\epsilon}\right\}$, in the case where the limit belongs to $W_{p}$ and that $\boldsymbol{E} \sup _{s \leq t} \rho\left(0, y_{s / \epsilon}^{\epsilon}\right)^{p}$ is uniformly bounded in $\epsilon$, implies their convergence in the Wasserstein $p$ distance. Thus, if $G$ is 
compact or has negative curvature and s.t. every point is a pole, our processes converge in the Wasserstein distance. The compact case is trivial. For the latter just note that in the proof of the lemma, $\tilde{\rho}^{2}$ can be replaced by $\rho^{2}$ and the left invariant vector fields are bounded. Since the sectional curvature is bounded, $\left|\nabla d \rho^{2}\right|$ is bounded by curvature comparison theorem. Thus $\rho\left(0, y_{s / \epsilon}^{\epsilon}\right)^{2}$ has finite moments, the assertion follows.

\subsection{Rate of convergence.}

The definition of $B C^{r}$ functions on a manifold depend on the linear connection on $T M$, in general. We use the flat connection $\nabla^{L}$ for $B C^{r}$ functions on the Lie group $G$. The canonical connection $\nabla^{c}$ is a convenient connection for defining $B C^{r}$ functions on $M=G / H$, as the parallel transports for $\nabla^{c}$ are differentials of the left actions of $G$ on $M$. See Section 10 below for more discussions on the canonical connection. In the theorem below, $M$ is given the induced $G$-invariant Riemannian metric. Set

$$
\begin{aligned}
& B C^{r}(M ; \mathbb{R})=\left\{f \in C^{r}(M ; \mathbb{R}):|f|_{\infty}+|\nabla f|_{\infty}+\sum_{k=1}^{r}\left|\left(\nabla^{c}\right)^{(k)} d f\right|_{\infty}<\infty\right\} \\
& B C^{r}(G ; \mathbb{R})=\left\{f \in C^{r}(G ; \mathbb{R}):|f|_{\infty}+|\nabla f|_{\infty}+\sum_{k=1}^{r}\left|\left(\nabla^{L}\right)^{(k)} d f\right|_{\infty}<\infty\right\} .
\end{aligned}
$$

Denote $|f|_{r, \infty}=\sum_{k=0}^{r}\left|\nabla^{(k)} d f\right|_{\infty}$ where $\nabla$ is one of the connections above.

REMARK 6.4. A function $f$ belongs to $B C^{r}(G)$ if and only if for an orthonormal basis $\left\{Y_{i}\right\}$ of $\mathfrak{g}$, the following functions are bounded for any set of indices: $f, Y_{i_{1}}^{*} f, \ldots$, $Y_{i_{1}}^{*} \ldots Y_{i_{r}}^{*} f$. In fact $\nabla f=\sum_{i} d f\left(Y_{i}^{*}\right) Y_{i}^{*}$ and $\nabla^{L} \nabla f=\sum_{i} Y_{i}^{*} L .\left(Y_{i}^{*} f\right)$, and so on. Here $L$. denotes Lie differentiation so $L . f=d f(\cdot)$.

Denote by $\bar{\mu}$ the probability measure of $\left(\bar{u}_{\text {. }}\right)$ and $\bar{P}_{t}$ the associated probability semigroup. We give the rate of convergence, which essentially follows from [47, Theorem 7.2], to follow which we would assume one of the following conditions. (1) $G$ is compact; (2) For some point $o \in G, \rho_{o}^{2}$ is smooth and $\left|\nabla^{L} d \rho_{o}^{2}\right| \leq C+K \rho_{o}^{2}$. (3) There exist $V \in C^{2}\left(M ; \mathbb{R}_{+}\right)$, $c>0, K>0$ and $q \geq 1$ such that

$$
|\nabla V| \leq C+K V, \quad|\nabla d V| \leq c+K V, \quad\left|\nabla d \rho_{o}^{2}\right| \leq c+K V^{q} .
$$

However it is better to prove the rate of convergence directly.

THEOREM 6.5. Suppose that $H$ is compact and $\left\{A_{1}, \ldots, A_{N}\right\}$ is Lie algebra generating and $\bar{Y}_{0}=0$. Then,

(1) Both $\left(u_{t / \epsilon}^{\epsilon}, t \leq T\right)$ and $\left(x_{t / \epsilon}^{\epsilon}, t \leq T\right)$ converge in $W_{p}$ for any $p>1$.

(2) There are numbers c such that for all $f \in B C^{4}(G ; \mathbb{R})$,

$$
\sup _{0 \leq t \leq T}\left|\boldsymbol{E} f\left(u_{t / \epsilon}^{\epsilon}\right)-\bar{P}_{t} f\left(u_{0}\right)\right| \leq C \epsilon \sqrt{|\log \epsilon|}\left(1+|f|_{4, \infty}\right), \quad u_{0} \in G .
$$

(3) For any $r \in(0,1 / 4), \sup _{0 \leq t \leq T} W_{1}\left(\operatorname{Law}\left(u_{t / \epsilon}^{\epsilon}\right), \bar{\mu}_{t}\right) \leq C \epsilon^{r}$. An analogous statement holds for $x_{t / \epsilon}^{\epsilon}$. 
Proof. If $\rho^{2}$ is bounded or smooth, $L_{Y_{i}^{*}} L_{Y_{j}^{*}} \rho=\nabla^{L} d \rho\left(Y_{i}^{*}, Y_{j}^{*}\right)+\nabla_{Y_{i}^{*}}^{L} Y_{j}^{*}$ is bounded. Denote $\mathcal{T}^{L}$ the torsion of $\nabla^{L}$ and $\nabla$ the Levi-Civita connection. The derivative flow for (6.1) satisfying the following equation

$$
\nabla v_{t}^{\epsilon}=-\frac{1}{2} \sum_{j} \alpha\left(Y_{0}, Y_{j}\right)\left(h_{t}^{\epsilon}\right) \mathcal{T}^{L}\left(v_{t}^{\epsilon}, Y_{j}\left(u_{t}^{\epsilon}\right)\right) .
$$

Indeed this follows from linearising the equation $\dot{u}_{t}^{\epsilon}=\sum_{j=1}^{N} Y_{j}^{*}\left(u_{t}^{\epsilon}\right) \alpha\left(Y_{0}, Y_{j}\right)\left(h_{t / \epsilon}\right)$ and the relation $\nabla^{L}=\nabla+(1 / 2) \mathcal{T}^{L}$. Since $\mathcal{T}^{L}(u, v)=-[u, v]$, the torsion tensor and their covariant derivatives are bounded. Thus all covariant derivatives of $Y_{j}^{*}$ with respect to the Levi-Civita connection are bounded. We use Lemma 6.2 and follow the proof of $[\mathbf{4 7}$, Theorem 7.2], to conclude the first assertion for the $u$ process. These are proved by discretising time and writing the differences as telescopic sums. The main ingredients are: $(1)\left(h_{t}\right)$ has an exponential mixing rate which follows from Hörmander's conditions, (2) estimates for $\left|\bar{P}_{t} f-\bar{P}_{s} f-(t-s) \bar{P}_{s} \overline{\mathcal{L}} f\right| \leq C(t-s)^{2}$ which follows from the fact that the vector fields $Y_{j}$ have bounded derivatives of all order.

For the process on the homogeneous manifold, take $f \in B C^{4}(M ; \mathbb{R})$ and let $\tilde{f}=$ $f \circ \pi$. Then $Y_{i} Y_{j} \tilde{f}=\nabla^{c} d f\left(\pi_{*} Y_{i}^{*}, \pi_{*} Y_{j}^{*}\right)+d f\left(\nabla_{Y_{i}^{*}}^{c} d \pi\left(Y_{j}^{*}\right)\right)$. The last term vanishes, as $(d \pi)_{u}\left(Y_{i}^{*}\right)=T \bar{L}_{u}\left((d \pi)_{1} Y_{i}\right)$, c.f. Lemma 10.2 below. Since $\pi_{*}$ is a Riemannian isometry, $Y_{i}^{*} Y_{j}^{*} \tilde{f}$ is bounded if $\nabla^{c} d f$ is. The same assertion holds for higher order derivatives. Thus $f \circ \pi \in B C^{4}(G ; \mathbb{R})$ and

$$
\sup _{0 \leq t \leq T}\left|\boldsymbol{E} f\left(x_{t / \epsilon}^{\epsilon}\right)-\pi_{*} \bar{P}_{t} f\left(x_{0}\right)\right| \leq C \epsilon \sqrt{|\log \epsilon|} \gamma\left(x_{0}\right)\left(1+|f|_{4, \infty}\right),
$$

where $\gamma$ is a function in $B_{\rho, o}$.

For the convergence in the Wasserstein distance, denote by $\tilde{\rho}$ and $\rho$ respectively the Riemannian distance function on $G$ and on $M$. For $i=1,2$, let $x_{i} \in M$ and $u_{i} \in \pi^{-1}\left(x_{i}\right)$. If $u_{1}$ and $u_{2}$ are the end points of a horizontal lift of the unit speed geodesic connecting $x_{1}$ and $x_{2}$, then $\tilde{\rho}\left(u_{1}, u_{2}\right)=\rho\left(x_{1}, x_{2}\right)$. Otherwise $\tilde{\rho}\left(u_{1}, u_{2}\right) \geq \rho\left(x_{1}, x_{2}\right)$. If $c_{1}$ and $c_{2}$ are $C^{1}$ curves on $M$ with $c^{1}$ curves $\tilde{c}_{1}$ and $\tilde{c}_{2}$ on $G$ covering $c_{1}$ and $c_{2}$ respectively, then $\rho\left(c_{1}, c_{2}\right)=\sup _{t \in[0, T]} \rho\left(c_{1}(t), c_{2}(t)\right) \leq \tilde{\rho}\left(\tilde{c}_{1}, \tilde{c}_{2}\right)$.

Since $C_{u_{0}}([0, T] ; G)$ is a Polish space, there is an optimal coupling of the probability law of $u_{\cdot / \epsilon}^{\epsilon}$ and $\bar{\mu}$ which we denote by $\mu$. Then $\pi_{*} \bar{\mu}$ is a coupling of $\operatorname{Law}\left(x_{\cdot / \epsilon}^{\epsilon}\right)$ and $\pi_{*} \bar{\mu}$ and

$$
\begin{aligned}
& W_{p}\left(\operatorname{Law}\left(u_{\cdot / \epsilon}^{\epsilon}\right), \bar{\mu}\right) \\
= & \left(\int_{C_{u_{0}}([0, T] ; G)} \tilde{\rho}^{p}\left(\gamma_{1}, \gamma_{2}\right) d \mu\left(\gamma_{1}, \gamma_{2}\right)\right)^{1 / p}\left(\int_{C_{x_{0}}([0, T] ; M)} \rho^{p}\left(\pi\left(\gamma_{1}\right), \pi\left(\gamma_{2}\right)\right) d \mu\left(\gamma_{1}, \gamma_{2}\right)\right)^{1 / p} \\
= & \left(\int_{C_{x_{0}}([0, T] ; M)} \sup _{0 \leq t \leq T} \rho^{p}\left(\sigma_{1}(t), \sigma_{2}(t)\right) d \pi_{*} \bar{\mu}\left(\sigma_{1}, \sigma_{2}\right)\right)^{1 / p} \\
\geq & W_{p}\left(\operatorname{Law}\left(x_{\cdot / \epsilon}^{\epsilon}\right), \pi_{*} \bar{\mu}\right) .
\end{aligned}
$$

Consequently $x_{\cdot / \epsilon}^{\epsilon}$ converges in $W_{p}$. Similarly the rate of convergence passes from the 
$u$ process to the $x$ process. For $r<1 / 4$ there is a number $C$ such that, $W_{1}\left(\operatorname{Law}\left(x_{t / \epsilon}^{\epsilon}\right)\right.$, $\left.\pi_{*} \bar{\mu}_{t}\right) \leq W_{p}\left(\operatorname{Law}\left(u_{t / \epsilon}^{\epsilon}\right), \bar{\mu}\right) \leq C \gamma\left(x_{0}\right) \epsilon^{r}$.

\subsection{The centre condition.}

We identify those vectors $Y_{0}$ satisfying $\bar{Y}_{0}=0$. Given a reductive structure, $\operatorname{Ad}_{H}$ is the direct sum of sub representations $\operatorname{Ad}_{H}=\left.\left.\operatorname{Ad}_{H}\right|_{\mathfrak{h}} \oplus \operatorname{Ad}_{H}\right|_{\mathfrak{m}}$. The condition $\operatorname{Ad}(H)(\mathfrak{m}) \subset \mathfrak{m}$ implies that $[\mathfrak{h}, \mathfrak{m}] \subset \mathfrak{m}$ and $\operatorname{Ad}(H)(\mathfrak{m})=\mathfrak{m}$. Let

$$
\mathfrak{m}_{0}=\{X \in \mathfrak{m}: \operatorname{Ad}(h)(X)=X \text { for all } h \in H\},
$$

be the subspace on which $\operatorname{Ad}_{H}$ acts trivially. We consider an $\operatorname{Ad}_{H}$-invariant subspace space $\tilde{\mathfrak{m}}$ of $\mathfrak{m}$, transversal to $\mathfrak{m}_{0}$, i.e. $\tilde{\mathfrak{m}} \cap \mathfrak{m}_{0}=\{0\}$. It is more intuitive to study the action of $\operatorname{Ad}_{H}$ through its isotropy representation $\tau$ of $H$ on $T_{o} M$ which is defined by the formula $\tau_{h}\left(\pi_{*} X\right):=\left(\bar{L}_{h}\right)_{*}\left(\pi_{*} X\right)$. Since left translation on $T_{o} M$ corresponds to adjoint action on $\mathfrak{m}$, then $\pi_{*} \operatorname{Ad}(h)(X)$ agree with $\left(\bar{L}_{h}\right)_{*}\left(\pi_{*} X\right)$, the linear representation $\operatorname{Ad}_{H}$ is equivalent to the isotropy representation. A representation $\rho$ of $H$ is said to acts transitively on the unit sphere, of the representation space $V$, if for any two unit vectors in $V$ there is a $\rho(h)$ taking one to the other. If $\operatorname{Ad}_{H}$ acts transitively, its representation space is irreducible and $\mathfrak{m}_{0}=\{0\}$.

Recall that $H$ is unimodular is equivalent to that the Haar measure $d h$ is bi-invariant.

Lemma 6.6. Suppose that $H$ is uni-modular.

(1) If $Y \in \mathfrak{m}_{0}$ is non-trivial, then $\bar{Y}$ does not vanish.

(2) $\bar{Y}=0$ for every $Y \in \tilde{\mathfrak{m}}$. In particular, if $\left.\operatorname{Ad}_{H}\right|_{\mathfrak{m}}$ has no non-trivial invariant vectors, then $\bar{Y}=0$ for all $Y \in \mathfrak{m}$.

Proof. Part (1) is clear by the definition. For any $Y \in \tilde{\mathfrak{m}}$, the integral $\bar{Y}:=$ $\int_{H} \operatorname{Ad}(h)(Y) d h$ is an invariant vector of $\operatorname{Ad}_{H}$, using the bi-invariance of the measure $d h$. Since $\bar{Y} \in \tilde{\mathfrak{m}}$ and $\tilde{\mathfrak{m}} \cap\left\{\mathfrak{m}_{0}\right\}=\{0\}$ the conclusion follows.

Example 6.7. Let $M$ be the Stiefel manifold $S(k, n)$ of oriented $k$ frames in $\mathbb{R}^{n}$. The orthogonal group takes a $k$-frame to a $k$ frame and acts transitively. The isotropy group of the $k$-frame $o=\left(e_{1}, \ldots, e_{k}\right)$, the first $k$ vectors from the standard basis of $\mathbb{R}^{n}$, contains rotation matrices that keep the first $k$ frames fixed and rotates the rest. Hence, $S(k, n)=S O(n) / S O(n-k)$. Then $\mathfrak{h}=\left\{\left(\begin{array}{ll}0 & 0 \\ 0 & A\end{array}\right)\right\}$ where $A \in \mathfrak{s o}(n-k)$. Denote by $M_{n-k, k}$ the set of $(n-k) \times k$ matrices and let $\mathfrak{m}=\left\{\left(\begin{array}{c}S \\ C\end{array} C^{T}\right)\right\}$ where $S \in \mathfrak{s o}(k)$ and $C \in M_{n-k, k}$. Since $\operatorname{Ad}\left(\left(\begin{array}{ll}1 & 0 \\ 0 & R\end{array}\right)\right)\left(\left(\begin{array}{cc}S & -C^{T} \\ C & 0\end{array}\right)\right)=\left(\begin{array}{cc}S & -C^{T} R^{T} \\ R C & 0\end{array}\right)$ for $R \in S O(n-k)$, we see $\mathfrak{m}_{0}=\left\{\left(\begin{array}{ll}S & 0 \\ 0 & 0\end{array}\right)\right\}$. Define $\tilde{\mathfrak{m}}=\left\{\left(\begin{array}{cc}0 & -C^{T} \\ C & 0\end{array}\right)\right\}$. Let us identify $\tilde{\mathfrak{m}}$ with $(n-k) \times k$ matrices. For $C \in M_{n-k, k}$ denote by $Y_{0}$ the corresponding skew symmetric matrix in $\tilde{m}$. It is clear that $\int_{H} R C d R$ and $\int_{H} \operatorname{Ad}(R)\left(Y_{0}\right) d R$ vanish.

The symmetry group of a Riemannian homogeneous space of dimension $d$ has at most dimension $d(d+1) / 2$. If $G / H$ is a connected $d$-dimensional manifold and if $G$ admits an $\operatorname{Ad}_{H}$-invariant inner product with $\operatorname{dim}(G)=(1 / 2) d(d+1)$, then $\bar{Y}$ vanishes for all $Y \in \mathfrak{m}$. Such Riemannian homogenous manifolds are of constant curvature, isometric to 
one of the following spaces: an Euclidean space, a sphere, a real projective space, and a simply connected hyperbolic space.

Example 6.8. Suppose that $M=G / H$ is a symmetric space, it is in particular a reductive homogeneous space. Then $L_{h} \pi=\pi \operatorname{Ad}(h)$ and $\left(d L_{h}\right)_{o} d \pi=d \pi \operatorname{Ad}_{h}$, where $o$ is the identity coset. Since $d \pi$ restricts to an isomorphism on $\mathfrak{m}$, left actions correspond to the Ad action on $G$. For every $Y_{0}$ there is an $h$ with the property that $\operatorname{Ad}(h)\left(Y_{0}\right)=-Y_{0}$. A compact Lie group with the bi-invariant metric is a symmetric space.

ExAmple 6.9. A more general class of manifolds, for which $\bar{Y}_{0}=0$ for every $Y_{0} \in \mathfrak{m}$, are weakly symmetric spaces among the Riemannian homogeneous spaces. A Riemannian manifold is weakly symmetric if there exists some closed subgroup $K$ of the Isometry group $I(M)$ and some $\mu \in I(M)$ such that $\mu^{2} \in K$ and for all $p, q \in M$ there exists $I \in K$ such that $I(p)=\mu(q)$ and $I(q)=\mu(p)$. This concept was introduced in Selberg [63] and studied by Szabó who characterises them as ray symmetric space. Also, a Riemannian manifold is weakly symmetric if and only if any two points in it can be interchanged by some isometry, see Berndt and Vanhecke [6]. The following property characterizes weakly symmetric spaces among Riemannian homogeneous spaces: let $H$ be the isotropy subgroup of $I(M)$ and $\tau: H \rightarrow G L\left(T_{o} M\right)$ its isotropy representation. Then for each $X \in \mathfrak{h}$ there exists an element of $h \in H$ such that $\rho(h)(X)=-X$. Some of the weakly symmetric spaces are given in the form of $G / H$ where $G$ is a connected semi simple Lie group, $K$ a closed subgroup of $G$, and $H$ a closed subgroup $K$. Then there is a principal fibration $G / H \rightarrow G / K$ with fibre $K / H$.

The three spheres with the left invariant Berger's metrics, $\epsilon \neq 1$, are not locally Riemannian symmetric spaces, they are reductive homogeneous spaces. We remark also that irreducible symmetric spaces are in fact strongly isotropy irreducible. A connected homogeneous manifold $G / H$ is strongly isotropy irreducible if $H$ is compact and its identity component acting irreducibly on the tangent space. Such spaces admit left invariant Einstein metric and are completely classified, for more study see [7] and see also [12]. If the symmetric space has rank 1, i.e. it is the quotient space of a semisimple Lie group $G$ whose maximal torus group $H$ is of dimension 1, they are two point homogeneous spaces, see [30, Proposition 5.1].

TheOrem 6.10. Suppose that $H$ is compact and $\left\{A_{0}, A_{1}, \ldots, A_{N}\right\}$ is Lie algebra generating. Let $Y_{0} \in \tilde{\mathfrak{m}}, x_{t}^{\epsilon}=\pi\left(g_{t}^{\epsilon}\right)$, where $\left(g_{t}^{\epsilon}\right)$ is the solution to (1.1), and $\left(u_{t}^{\epsilon}\right)$ its horizontal lift through $g_{0}$. Then $\left(u_{t / \epsilon}^{\epsilon}\right)$ and $\left(x_{t / \epsilon}^{\epsilon}\right)$ converge weakly with respective limit diffusion process $\left(\bar{u}_{t}\right)$ and $\left(\bar{x}_{t}\right)$. Let $\left\{Y_{j}, j=1, \ldots, N\right\}$ be an orthonormal basis of $\tilde{\mathfrak{m}}$. Then the Markov generator of $\left(\bar{u}_{t}\right)$ is

$$
\overline{\mathcal{L}}=-\sum_{i, j=1}^{N} a_{i, j}\left(Y_{0}\right) L_{Y_{i}^{*}} L_{Y_{j}^{*}}, \quad a_{i, j}\left(Y_{0}\right)=\int_{H} \alpha\left(Y_{0}, Y_{i}\right) \mathcal{L}_{0}^{-1}\left(\alpha\left(Y_{0}, Y_{j}\right)\right) d h .
$$

Proof. By Corollary 4.4 the horizontal lift process of $\left(x_{t}^{\epsilon}\right)$ solves equations (6.1)(6.2). By Lemma 6.6, $\bar{Y}_{0}=0$. Since $\tilde{\mathfrak{m}}$ is an invariant space of $\operatorname{Ad}_{H}$ and $Y_{0} \in \tilde{\mathfrak{m}}$, $\operatorname{Ad}(h)\left(Y_{0}\right) \in \tilde{\mathfrak{m}}$. In Proposition 6.2 it is sufficient to take $\left\{Y_{j}\right\}$ to be an orthonormal 
basis of the invariant subspace $\tilde{\mathfrak{m}}$.

In the rest of the paper we study the effective limits $\overline{\mathcal{L}}$ given in Theorem 6.10 . Although $\bar{u}_{t}$ is always a Markov process, that its projection $\left(\bar{x}_{t}\right)$ (describing the motion of the effective orbits) is a Markov process on its own is not automatic. In the next two sections we study this and identify the stochastic processes $\left(\bar{u}_{t}\right)$ and $\left(\bar{x}_{t}\right)$ by computing the Markov generator $\overline{\mathcal{L}}$ and its projection.

\section{Effective limits, Casimir, and Markov property.}

In this section $H$ is a compact connected proper subgroup of $G, \mathcal{L}_{0}=$ $(1 / 2) \sum_{k=1}^{N}\left(A_{k}\right)^{2},\langle$,$\rangle an \operatorname{Ad}_{H}$-invariant inner product on $\mathfrak{g}$ and $\mathfrak{m}=\mathfrak{h}^{\perp}$. Let $\mathfrak{m}=$ $\mathfrak{m}_{0} \oplus \mathfrak{m}_{1} \oplus \cdots \oplus \mathfrak{m}_{r}$ be the orthogonal sum of $\operatorname{Ad}_{H}$-invariant subspaces, where each $\mathfrak{m}_{j}$ is irreducible for $j \neq 0$. This is also an invariant decomposition for $\operatorname{ad}_{\mathfrak{h}}$.

Let $\mathcal{B}_{\mathrm{ad}_{\mathfrak{h}}, \mathfrak{m}_{l}}(X, Y)$ be the symmetric associative bilinear form of the adjoint subrepresentation of $\mathfrak{h}$ on $\mathfrak{m}_{l}$. The symmetric bilinear form for a representation $\rho$ of a Lie algebra $\mathfrak{g}$ on a finite dimensional vector space is given by the formula $\mathcal{B}_{\rho}(X, Y)=$ trace $\rho(X) \rho(Y)$ for $X, Y \in \mathfrak{h}$. A bilinear form $\mathcal{B}$ on a Lie algebra is associative if $\mathcal{B}([X, Y], Z)=\mathcal{B}(X,[Y, Z])$. Let $\operatorname{Id}_{\mathfrak{m}_{l}}$ be the identity map on $\mathfrak{m}_{l}$. The following lemma allows us to compute the coefficients of the generator.

Lemma 7.1. Let $\left\{A_{j}\right\}$ be an orthonormal basis of $\mathfrak{h}$. The following statements hold.

1. There exists $\lambda_{l}$ such that $\left.(1 / 2) \sum_{k=1}^{p} \operatorname{ad}^{2}\left(A_{k}\right)\right|_{\mathfrak{m}_{l}}=-\lambda_{l} \operatorname{Id}_{\mathfrak{m}_{l}}$. Furthermore $\lambda_{l}=0$ if and only if $l=0$.

2. Suppose that $\operatorname{ad}_{\mathfrak{h}}: \mathfrak{h} \rightarrow \mathbb{L}\left(\mathfrak{m}_{l} ; \mathfrak{m}_{l}\right)$ is faithful. Then $\mathcal{B}_{\mathrm{ad}_{\mathfrak{h}}, \mathfrak{m}_{l}}$ is non-degenerate, negative definite, and $\operatorname{Ad}_{H}$-invariant. If $l \neq 0$,

$$
\lambda_{l}=-\mathcal{B}_{\operatorname{ad}_{h}, \mathfrak{m}_{l}}(X, X) \frac{\operatorname{dim}(\mathfrak{h})}{2 \operatorname{dim}\left(\mathfrak{m}_{l}\right)},
$$

where $X$ is any unit vector in $\mathfrak{h}$. If the inner product on $\mathfrak{h}$ agrees with $-\mathcal{B}_{\mathrm{ad}_{h}, \mathfrak{m}_{l}}$ then $\lambda_{l}=\operatorname{dim}(\mathfrak{h}) / 2 \operatorname{dim}\left(\mathfrak{m}_{l}\right)$.

Proof. Part (1). For $Z \in \mathfrak{h}$ and $X, Y \in \mathfrak{g}$, we differentiate the identity

$$
\langle\operatorname{Ad}(\exp (t Z))(X), \operatorname{Ad}(\exp (t Z))(Y)\rangle=\langle X, Y\rangle
$$

at $t=0$ to see that

$$
\langle\operatorname{ad}(Z)(X), Y\rangle+\langle X, \operatorname{ad}(Z)(Y)\rangle=0
$$

Let $\left\{A_{1}, \ldots, A_{p}\right\}$ be an orthonormal basis of $\mathfrak{h}$, for the $\operatorname{Ad}_{H}$ invariant metric, then $\sum_{k=1}^{p}\left(\operatorname{ad}\left(A_{k}\right)\right)^{2}$ commutes with every element of $\mathfrak{h}$. Indeed if $X \in \mathfrak{h}$ let $\left[X, A_{k}\right]=$ $\sum_{l^{\prime}} a_{k l^{\prime}} A_{l^{\prime}}$, then

$$
a_{k l}=\sum_{l^{\prime}} a_{k, l^{\prime}}\left\langle A_{l^{\prime}}, A_{l}\right\rangle=\left\langle\left[X, A_{k}\right], A_{l}\right\rangle=-\left\langle\left[X, A_{l}\right], A_{k}\right\rangle=-a_{l k},
$$




$$
\begin{aligned}
{\left[\operatorname{ad}(X), \sum_{k=1}^{p} \operatorname{ad}^{2}\left(A_{k}\right)\right] } & =\sum_{k}\left[\operatorname{ad}(X), \operatorname{ad}\left(A_{k}\right)\right] \operatorname{ad}\left(A_{k}\right)+\sum_{k} \operatorname{ad}\left(A_{k}\right)\left[\operatorname{ad}(X), \operatorname{ad}\left(A_{k}\right)\right] \\
& =\sum_{k} \operatorname{ad}\left(\left[X, A_{k}\right]\right) \operatorname{ad}\left(A_{k}\right)+\sum_{k} \operatorname{ad}\left(A_{k}\right) \operatorname{ad}\left(\left[X, A_{k}\right]\right)=0 .
\end{aligned}
$$

If $Y_{0} \in \mathfrak{m}, \operatorname{ad}\left(A_{k}\right)\left(Y_{0}\right) \in \mathfrak{m}$ and by the skew symmetry, (7.1),

$$
\left\langle\sum_{k=1}^{p}\left(\operatorname{ad}\left(A_{k}\right)\right)^{2}\left(Y_{0}\right), Y_{0}\right\rangle=-\sum_{k=1}^{p}\left\langle\operatorname{ad}\left(A_{k}\right)\left(Y_{0}\right), \operatorname{ad}\left(A_{k}\right)\left(Y_{0}\right)\right\rangle .
$$

Thus $\sum_{k=1}^{p}\left(\operatorname{ad}\left(A_{k}\right)\right)^{2}\left(Y_{0}\right)=0$, where $p=\operatorname{dim}(\mathfrak{h})$, implies that $\operatorname{ad}\left(A_{k}\right)\left(Y_{0}\right)=0$ for all $k$ which in turn implies that $Y_{0} \in \mathfrak{m}_{0}$. Conversely if $Y_{0} \in \mathfrak{m}_{0}$ it is clear that $\sum_{k=1}^{p} \operatorname{ad}^{2}\left(A_{k}\right)=0$.

(2) Firstly, $\mathcal{B}_{\mathrm{ad}_{\mathfrak{h}}, \mathfrak{m}_{l}}$ is non-degenerate. If $\left\{Y_{j}\right\}$ is a basis of $\mathfrak{m}_{l}$ with respect to the $\operatorname{ad}_{H}$ invariant inner product and $X \in \mathfrak{h}$, then

$$
\mathcal{B}_{\mathrm{ad}_{\mathfrak{h}}, \mathfrak{m}_{l}}(X, X)=-\sum_{j}\left|\left[X, Y_{j}\right]\right|^{2},
$$

which vanishes only if $\left[X, Y_{j}\right]=0$ for all $j$. By the skew symmetry of $\operatorname{ad}_{\mathfrak{h}}$, for any $Y \in \mathfrak{m}_{l}$, $0=\left\langle\left[X, Y_{j}\right], Y\right\rangle=-\left\langle Y_{j},[X, Y]\right\rangle$ for all $j$. Since $\operatorname{ad}(X)\left(\mathfrak{m}_{l}\right) \subset \mathfrak{m}_{l}, \mathcal{B}_{\operatorname{ad}_{\mathfrak{h}}, \mathfrak{m}_{l}}(X, X)=0$ implies that $[X, Y]=0$ for all $Y$ which implies $X$ vanishes from the assumption that $\operatorname{ad}_{\mathfrak{h}}$ is faithful. It is clear that $\mathcal{B}_{\mathrm{ad}_{h}, \mathfrak{m}_{l}}$ is $\operatorname{Ad}_{H}$-invariant. Let $X \in \mathfrak{h}$ and $h \in H$, then

$$
\begin{aligned}
& \mathcal{B}_{\operatorname{ad}_{\mathfrak{h}}, \mathfrak{m}_{l}}(\operatorname{Ad}(h)(X), \operatorname{Ad}(h)(X))=\sum_{j}\left\langle\operatorname{ad}(\operatorname{Ad}(h)(X)) \operatorname{ad}(\operatorname{Ad}(h)(X)) Y_{j}, Y_{j}\right\rangle \\
& =-\sum_{j}\left\langle\left[\operatorname{Ad}(h)(X), Y_{j}\right],\left[\operatorname{Ad}(h)(X), Y_{j}\right]\right\rangle \\
& =-\sum_{j}\left\langle\operatorname{Ad}(h)\left[X, \operatorname{Ad}\left(h^{-1}\right) Y_{j}\right], \operatorname{Ad}(h)\left[X, \operatorname{Ad}\left(h^{-1}\right) Y_{j}\right]\right\rangle \\
& =-\sum_{j}\left\langle\left[X, \operatorname{Ad}\left(h^{-1}\right) Y_{j}\right],\left[X, \operatorname{Ad}\left(h^{-1}\right) Y_{j}\right]\right\rangle=\mathcal{B}_{\operatorname{ad}_{\mathfrak{h}}, \mathfrak{m}_{l}}(X, X) .
\end{aligned}
$$

Since there is a unique, up to a scalar multiple, $\mathrm{Ad}_{H}$-invariant inner product on a compact manifold, $\mathcal{B}_{\mathrm{ad}_{\mathfrak{h}}, \mathfrak{m}_{l}}$ is essentially the inner product on $H$. There is a positive number $a_{l}$ such that $\mathcal{B}_{\mathrm{ad}_{\mathfrak{h}}, \mathfrak{m}_{l}}=-a_{l}\langle$,$\rangle . It is clear that a_{l}>0$. For the orthonormal basis $\left\{A_{k}\right\}$, $\mathcal{B}_{\mathrm{ad}_{\mathfrak{h}}, \mathfrak{m}_{l}}\left(A_{i}, A_{j}\right)=-a_{l} \delta_{i, j}$. We remark that $\left\{\left(1 / a_{l}\right) A_{k}\right\}$ is a dual basis of $\left\{A_{k}\right\}$ with respect to $\mathcal{B}_{\mathrm{ad}_{\mathfrak{h}}, \mathfrak{m}_{l}}$ and $\left(1 / a_{l}\right) \sum_{k} \operatorname{ad}^{2}\left(A_{k}\right)$ is the Casimir element.

By part (1), there is a number $\lambda_{l}$ such that $(1 / 2) \sum_{k=1}^{\operatorname{dim}(\mathfrak{h})} \operatorname{ad}^{2}\left(A_{k}\right)=-\lambda_{l} \operatorname{Id}_{\mathfrak{m}_{l}}$. The ration between the symmetric form $\mathcal{B}_{\mathrm{ad}_{\mathfrak{h}}, \mathfrak{m}_{l}}$ and the inner products on $\mathfrak{m}_{l}$ can be determined by any unit length vector in $\mathfrak{h}$. It follows that

$$
\operatorname{trace}_{\mathfrak{m}_{l}} \sum_{k=1}^{\operatorname{dim}(\mathfrak{h})} \operatorname{ad}^{2}\left(A_{k}\right)=\operatorname{trace}_{\mathfrak{m}_{l}}\left(-2 \lambda_{l} \operatorname{Id}_{\mathfrak{m}_{l}}\right)=-2 \lambda_{l} \operatorname{dim}\left(\mathfrak{m}_{l}\right) .
$$


On the other hand,

$$
\operatorname{trace}_{\mathfrak{m}_{l}}\left(\sum_{k=1}^{\operatorname{dim}(\mathfrak{h})} \operatorname{ad}^{2}\left(A_{k}\right)\right)=\sum_{k=1}^{\operatorname{dim}(\mathfrak{h})} \operatorname{trace}_{\mathfrak{m}_{l}}\left(\operatorname{ad}^{2}\left(A_{k}\right)\right)=\sum_{k=1}^{\operatorname{dim}(\mathfrak{h})} \mathcal{B}_{\operatorname{ad}_{h}, \mathfrak{m}_{l}}\left(A_{k}, A_{k}\right) .
$$

Consequently $\lambda_{l}=-\mathcal{B}_{\mathrm{ad}_{h}, \mathfrak{m}_{l}}\left(A_{1}, A_{1}\right) \operatorname{dim}(\mathfrak{h}) / 2 \operatorname{dim}\left(\mathfrak{m}_{l}\right)$. We completed part (2).

If $\mathfrak{h}=\mathfrak{h}_{0} \oplus \mathfrak{h}_{1}$ s.t. $\mathfrak{h}_{0}$ acts trivially on $\mathfrak{m}_{l}$ and $\mathfrak{h}_{1}$ a sub Lie-algebra acts faithfully, we take $\mathfrak{h}_{1}$ in place of $\mathfrak{h}, \lambda_{l}$ can be computed using the formula in (2) with $\left\{A_{k}\right\}$ taken to be an orthonormal basis of $\mathfrak{h}_{1}$.

If $\mathcal{A}=(1 / 2) \sum_{k=1}^{N}\left(X_{k}\right)^{2}+X_{0}$ where $X_{k} \in \mathfrak{g}$, we denote by $c(\mathcal{A})=$ $(1 / 2) \sum_{k=1}^{N} \operatorname{ad}^{2}\left(X_{k}\right)+\operatorname{ad}\left(X_{0}\right)$ to be the linear map on $\mathfrak{g}$.

Lemma 7.2. For any $Y_{0} \in \mathfrak{m}, \mathcal{L}_{0}\left(\operatorname{Ad}(\cdot) Y_{0}\right)=\operatorname{Ad}(\cdot)\left(c\left(\mathcal{L}_{0}\right)\left(Y_{0}\right)\right)$. If $Y_{0}$ is an eigenvector of $c\left(\mathcal{L}_{0}\right)$ corresponding to an eigenvalue $-\lambda\left(Y_{0}\right)$ then for any $Y \in \mathfrak{m}$, $\left\langle Y, \operatorname{Ad}(h)\left(Y_{0}\right)\right\rangle$ is an eigenfunction of $\mathcal{L}_{0}$ corresponding to $-\lambda\left(Y_{0}\right)$. The converse also holds.

Proof. Just note that

$$
L_{A_{k}}\left(\operatorname{Ad}(\cdot)\left(Y_{0}\right)\right)(h)=\left.\frac{d}{d t}\right|_{t=0} \operatorname{Ad}\left(h \exp \left(t A_{k}\right)\right)\left(Y_{0}\right)=\operatorname{Ad}(h)\left(\left[A_{k}, Y_{0}\right]\right),
$$

which by iteration leads to $L_{A_{k}} L_{A_{k}} \operatorname{Ad}(\cdot)\left(Y_{0}\right)=\operatorname{Ad}(h)\left(\left[A_{k},\left[A_{k}, Y_{0}\right]\right]\right)$ and to the required identity $\mathcal{L}_{0}\left(\operatorname{Ad}(\cdot) Y_{0}\right)=\operatorname{Ad}(\cdot) c\left(\mathcal{L}_{0}\right)\left(Y_{0}\right)$. Furthermore, for every $Y \in \mathfrak{m}$,

$$
\mathcal{L}_{0}\left(\left\langle\operatorname{Ad}(\cdot) Y_{0}, Y\right\rangle\right)=\left\langle\operatorname{Ad}(\cdot)\left(c\left(\mathcal{L}_{0}\right) Y_{0}\right), Y\right\rangle
$$

from which follows the statement about the eigenfunctions.

We take an orthonormal basis $\left\{Y_{j}\right\}$ of $\mathfrak{m}_{l}$ and for $Y_{0}$ fixed set $\alpha_{j}\left(Y_{0}\right)=$ $\left\langle\operatorname{Ad}(\cdot)\left(Y_{0}\right), Y_{j}\right\rangle$. If $f$ is a real valued function, set $\bar{f}=\int_{H} f d h$ and by $\mathcal{L}_{0}^{-1} f$ we denote a solution to the Poisson equation $\mathcal{L}_{0} u=f$. Also set $\overline{\mathcal{L}}=$ $-\sum_{i, j=1}^{m_{l}} \overline{\alpha_{j}\left(Y_{0}\right) \mathcal{L}_{0}^{-1}\left(\alpha_{i}\left(Y_{0}\right)\right)} L_{Y_{i}} L_{Y_{j}}$. Below we give an invariant formula for the limiting operator on $G$.

TheOREm 7.3. Suppose that $\left\{A_{1}, \ldots, A_{p}\right\}$ is a basis of $\mathfrak{h}$ and $Y_{0} \in \mathfrak{m}_{l}$ where $l \neq 0$. Then, for any orthonormal basis $\left\{Y_{1}, \ldots, Y_{d}\right\}$ of $\mathfrak{m}_{l}$,

$$
\overline{\mathcal{L}} f=\sum_{i, j=1}^{d} a_{i, j}\left(Y_{0}\right) \nabla^{L} d f\left(Y_{i}, Y_{j}\right), \quad f \in C^{2}(G ; \mathbb{R}),
$$

where $a_{i, j}\left(Y_{0}\right)=\left(1 / \lambda_{l}\right) \int_{H}\left\langle Y_{i}, \operatorname{Ad}(h) Y_{0}\right\rangle\left\langle Y_{j}, \operatorname{Ad}(h) Y_{0}\right\rangle d h, \lambda_{l}$ is an eigenvalue of $\mathcal{L}_{0}$, and $(-1 / 2) \sum_{k=1}^{p} \operatorname{ad}^{2}\left(A_{k}\right)=\lambda_{l} \operatorname{Id}_{\mathfrak{m}_{l}}$. Equivalently,

$$
\overline{\mathcal{L}} f=\frac{1}{\lambda_{l}} \int_{H} \nabla^{L} d f\left(\left(\operatorname{Ad}(h)\left(Y_{0}\right)\right)^{*},\left(\operatorname{Ad}(h)\left(Y_{0}\right)\right)\right) d h .
$$


Furthermore, $\bar{x}_{t}$ is a Markov process with Markov generator

$$
\overline{\mathcal{L}}(F \circ \pi)(u)=\frac{1}{\lambda_{l}} \int_{H}\left(\nabla^{c} d F\right)_{\pi(u)}\left(d \bar{L}_{u h} Y_{0}, d \bar{L}_{u h} Y_{0}\right) d h, \quad F \in C^{2}(M ; \mathbb{R}),
$$

where $\nabla^{c}$ be the canonical connection on the Riemannian homogeneous manifold.

Proof. For the left invariant connection, $\nabla_{Y_{i}}^{L} Y_{j}^{*}=0$ for any $i, j$, and so

$$
-\sum_{i, j=1}^{m} \overline{\alpha_{i}\left(Y_{0}\right)\left(\mathcal{L}_{0}^{-1} \alpha_{j}\left(Y_{0}\right)\right)} Y_{i}^{*} Y_{j}^{*} f=-\sum_{i, j=1}^{m} \overline{\alpha_{i}\left(Y_{0}\right)\left(\mathcal{L}_{0}^{-1} \alpha_{j}\left(Y_{0}\right)\right)} \nabla^{L} d f\left(Y_{i}^{*}, Y_{j}^{*}\right) .
$$

We can always take the $\operatorname{Ad}_{H}$ invariant inner product on $\mathfrak{h}$ w.r.t. which $\left\{A_{1}, \ldots, A_{p}\right\}$ is an orthonormal basis of $\mathfrak{h}$, and so Lemma 7.1 applies. By Lemma 7.1, $(1 / 2) c\left(\mathcal{L}_{0}\right)=-\lambda_{l} \operatorname{Id}_{\mathfrak{m}}$. For any $Y_{0}, Y \in \mathfrak{m}_{l}, \beta\left(Y, Y_{0}\right)(\cdot):=\left\langle Y, \operatorname{Ad}(\cdot) Y_{0}\right\rangle$ is an eigenfunction of $\mathcal{L}_{0}$ with eigenvalue $-2 \lambda_{l}$, using Lemma 7.2. Note also that $\lambda \neq 0$. Consequently,

$$
\overline{\alpha_{i}\left(Y_{0}\right)\left(\mathcal{L}_{0}^{-1} \alpha_{j}\left(Y_{0}\right)\right)}=-\frac{1}{\lambda_{l}} \int_{H}\left\langle Y_{i}, \operatorname{Ad}(h) Y_{0}\right\rangle\left\langle Y_{j}, \operatorname{Ad}(h)\left(Y_{0}\right)\right\rangle d h .
$$

The equivalent formula is obtained from summing over the basis of $\mathfrak{m}_{l}$ :

$$
\begin{aligned}
& \sum_{i, j} \alpha_{i, j}\left(Y_{0}\right) \nabla^{L} d f\left(Y_{i}^{*}(u), Y_{j}^{*}(u)\right) \\
& =\frac{1}{\lambda_{l}} \int_{H}\left(\nabla^{L} d f\right)_{u}\left(\left(\operatorname{Ad}(h)\left(Y_{0}\right)\right)^{*}(u),\left(\operatorname{Ad}(h)\left(Y_{0}\right)\right)^{*}(u)\right) d h .
\end{aligned}
$$

Finally we prove the Markov property of the projection of the effective process. For $g \in G$,

$$
\begin{aligned}
& \nabla^{L} d(F \circ \pi)\left(\left(\operatorname{Ad}(h)\left(Y_{0}\right)\right)^{*}(g),\left(\operatorname{Ad}(h)\left(Y_{0}\right)\right)^{*}(g)\right) \\
& =L_{\operatorname{Ad}(h)\left(Y_{0}\right)^{*}}\left(d F\left(d \pi\left(\left(\operatorname{Ad}(h) Y_{0}\right)^{*}\right)\right)\right)(g)=\nabla^{c} d F\left(d \pi\left(\operatorname{Ad}(h)\left(Y_{0}\right)^{*}\right), d \pi\left(\operatorname{Ad}(h)\left(Y_{0}\right)^{*}\right)\right) .
\end{aligned}
$$

The last step is due to part (4) of Lemma 10.2 in Section 10, from which we conclude that $d F\left(\nabla_{\pi_{*} \operatorname{Ad}(h)\left(Y_{0}\right)^{*}}^{c} d \pi\left(\left(\operatorname{Ad}(h) Y_{0}\right)^{*}\right)\right)$ vanishes. It is clear that,

$$
\begin{aligned}
& \int_{H}\left(\nabla^{c} d F\right)_{\pi(g)}\left(d \pi\left(\operatorname{Ad}(h)\left(Y_{0}\right)^{*}(g)\right), d \pi\left(\operatorname{Ad}(h)\left(Y_{0}\right)(g)\right)\right) d h \\
& =\int_{H}\left(\nabla^{c} d F\right)_{\pi(g)}\left(d \bar{L}_{g h} d \pi\left(Y_{0}\right), d \bar{L}_{g h} d \pi\left(Y_{0}\right)\right) d h .
\end{aligned}
$$

Since $d h$ is right invariant, the above formulation is independent of the choice of $g$ in $\pi^{-1}(x)$. That the stochastic process $\pi\left(u_{t}\right)$ is a Markov process follows from Dynkin's criterion which states that if $\left(u_{t}\right)$ is a Markov process with semigroup $P_{t}$ and if $\pi: G \rightarrow M$ is a map such that $P_{t}(f \circ \pi)(y)$ depends only on $\pi(y)$ then $\pi\left(u_{t}\right)$ is a Markov process. See e.g. Dynkin $[\mathbf{1 6}]$ and Rosenblatt [62].

REMARKs. If we take the $\operatorname{Ad}_{H}$ invariant product on $\mathfrak{h}$ to be the one for which 
$\left\{A_{k}\right\}$ is an o.n.b. then $\mathcal{L}_{0}=\Delta_{H}=\sum_{k=1}^{p}\left(A_{k}\right)^{2}$ which follows from $H$ being compact, c.f. Lemma 10.1. The operator $\overline{\mathcal{L}}$ in the above Lemma provides an example of a cohesive operator, as defined by Elworthy, LeJan and Li [19].

\section{Using symmetries.}

We fix an $\operatorname{Ad}_{H}$ invariant inner product on $\mathfrak{g}$, and as usual, set $\mathfrak{m}=\mathfrak{h} \mathfrak{h}^{\perp}$ w.r.t an $\operatorname{Ad}_{H}$ invariant inner product on $\mathfrak{g}$. Let $\mathfrak{m}=\mathfrak{m}_{0} \oplus \mathfrak{m}_{1} \oplus \cdots \oplus \mathfrak{m}_{k}$ be an orthogonal decomposition. By $\tilde{\mathfrak{m}}$ we denote an $\mathrm{Ad}_{H}$ invariant subspace of $\mathfrak{m}$ not containing any non-trivial $\mathrm{Ad}_{H^{-}}$ invariant vectors. In this section we explore the symmetries of the manifold $M$ to study the functions of the form $\left\langle\operatorname{Ad}(\cdot) Y_{0}, Y_{j}\right\rangle$ where $\left\{Y_{i}\right\}$ is an orthonormal basis of $\tilde{\mathfrak{m}}$.

Definition 8.1. We say that $\operatorname{Ad}_{H}$ acts quasi doubly transitively (on the unit sphere) of $\tilde{\mathfrak{m}}$ if for any orthonormal basis $\left\{Y_{i}\right\}$ of $\tilde{\mathfrak{m}}$ and for any pair of numbers $i \neq j$ there is $h^{i, j} \in H$ such that $\operatorname{Ad}\left(h^{i, j}\right)\left(Y_{i}\right)=Y_{i}$ and $\operatorname{Ad}\left(h^{i, j}\right)\left(Y_{j}\right)=-Y_{j}$.

The family of Riemannian manifold, with a lot of symmetry, are two-point homogeneous spaces by which mean for any two points $x_{1}, x_{2}$ and $y_{1}, y_{2}$ with $d\left(x_{1}, x_{2}\right)=d\left(y_{1}, y_{2}\right)$ sufficiently small, there exist an isometry taking $\left(x_{1}, x_{2}\right)$ to $\left(y_{1}, y_{2}\right)$. Such spaces were classified by H.-C. Wang (1952, Annals) to be isometric to a symmetric Riemannian space $\operatorname{Iso}^{\circ}(M) / K$ where $K$ is compact. They have constant sectional curvatures in odd dimensions and the non-compact spaces are all simply connected and homeomorphic to an Euclidean space. On a locally two point homogeneous space $\operatorname{Ad}_{H}$ acts transitively on the unit sphere, and acts quasi doubly transitively. If $(e, f)$ or orthogonal unit vectors at $T_{o} M$. Let $x=\exp _{o}(\delta e), x^{\prime}=\exp _{o}(-\delta e)$, and $y=\exp _{o}(\delta f)$ where $\delta$ is a number sufficiently small for them to be defined. There is an isometry $\phi$ taking $x$ to $y$ leaving $o$ fixed. This isometry taking the geodesic $0 x$ to the geodesic oy. It would take $y$ to $x^{\prime}$ which implies that $\rho(x, y)=\rho\left(y, x^{\prime}\right)$.

LEMMA 8.1. Let $\left\{Y_{1}, \ldots, Y_{d}\right\}$ be an orthonormal basis of $\tilde{\mathfrak{m}}$, an $\operatorname{Ad}_{H}$ invariant subspace of $\mathfrak{m}$ with $\tilde{\mathfrak{m}} \cap \mathfrak{m}_{0}=\{0\}$. Let $Y_{0} \in \tilde{\mathfrak{m}}$.

1. If $\operatorname{Ad}_{H}$ acts transitively on the unit sphere of $\tilde{\mathfrak{m}}$,

$$
\int_{H}\left\langle Y_{i}, \operatorname{Ad}(h) Y_{0}\right\rangle^{2} d h=\frac{\left|Y_{0}\right|^{2}}{\operatorname{dim}(\tilde{\mathfrak{m}})}, \quad i=1, \ldots, \operatorname{dim}(\tilde{\mathfrak{m}}) .
$$

2. If $\operatorname{Ad}_{H}$ acts quasi doubly transitively on $\tilde{\mathfrak{m}}$ then for $i \neq j$,

$$
\int_{H}\left\langle Y_{i}, \operatorname{Ad}(h) Y_{0}\right\rangle\left\langle Y_{j}, \operatorname{Ad}(h)\left(Y_{0}\right)\right\rangle d h=0 .
$$

3. $\operatorname{dim}(\tilde{\mathfrak{m}})>1$.

4. If $\operatorname{dim}(\tilde{\mathfrak{m}})=2$ then $\int_{H}\left\langle Y_{i}, \operatorname{Ad}(h) Y_{0}\right\rangle\left\langle Y_{j}, \operatorname{Ad}(h)\left(Y_{0}\right)\right\rangle d h=\left(\left|Y_{0}\right|^{2} / 2\right) \delta_{i, j}$ for all $i, j$.

Proof. Suppose that $\operatorname{Ad}\left(h_{0}\right)$ take $Y_{j}$ to $Y_{1}$. Then 


$$
\int_{H}\left\langle Y_{j}, \operatorname{Ad}(h) Y_{0}\right\rangle^{2} d h=\int_{H}\left\langle Y_{j}, \operatorname{Ad}\left(h_{0}^{-1}\right) \operatorname{Ad}(h)\left(Y_{0}\right)\right\rangle^{2} d h=\int_{H}\left\langle Y_{1}, \operatorname{Ad}(h)\left(Y_{0}\right)\right\rangle^{2} d h,
$$

using the $\operatorname{Ad}_{H}$-invariance of the Haar measure and the inner product. Set $d=\operatorname{dim}(\tilde{\mathfrak{m}})$. Since $\tilde{\mathfrak{m}}$ is an invariant space of $\operatorname{Ad}_{H}$ we sum over the basis vectors to obtain

$$
\sum_{j=1}^{d} \int_{H}\left\langle Y_{j}, \operatorname{Ad}(h) Y_{0}\right\rangle^{2} d h=\int_{H} \sum_{j=1}^{d}\left\langle\operatorname{Ad}\left(h^{-1}\right) Y_{j}, Y_{0}\right\rangle^{2} d h=\left|Y_{0}\right|^{2} .
$$

It follows that $\int_{H}\left\langle Y_{j}, \operatorname{Ad}(h) Y_{0}\right\rangle^{2} d h=\left|Y_{0}\right|^{2} / \operatorname{dim}(\tilde{\mathfrak{m}})$. For $i \neq j$, there exists $h^{i, j} \in H$ such that $\operatorname{Ad}\left(h^{i, j}\right)\left(Y_{i}\right)=Y_{i}$ and $\operatorname{Ad}\left(h^{i, j}\right)\left(Y_{j}\right)=-Y_{j}$. By the bi-invariance of the Haar measure and the $\mathrm{Ad}_{H}$-invariance of the inner product again we obtain,

$$
\begin{aligned}
& \int_{H}\left\langle Y_{i}, \operatorname{Ad}(h) Y_{0}\right\rangle\left\langle Y_{j}, \operatorname{Ad}(h) Y_{0}\right\rangle d h \\
& =\int_{H}\left\langle Y_{i}, \operatorname{Ad}\left(h^{i, j}\right) \operatorname{Ad}(h)\left(Y_{0}\right)\right\rangle\left\langle Y_{j}, \operatorname{Ad}\left(h^{i, j}\right) \operatorname{Ad}(h) Y_{0}\right\rangle d h \\
& =-\int_{H}\left\langle Y_{i}, \operatorname{Ad}(h)\left(Y_{0}\right)\right\rangle\left\langle Y_{j}, \operatorname{Ad}(h) Y_{0}\right\rangle d h
\end{aligned}
$$

and part (2) follows.

We observe that no $\mathrm{Ad}_{H^{-}}$-invariant subspace of $\mathfrak{m}$, containing no trivial $\mathrm{Ad}_{H^{-}}$ invariant vectors, can be one dimensional, for otherwise, every orthogonal transformation $\operatorname{Ad}(h)$ takes a $Y_{0} \in \tilde{\mathfrak{m}}$ to itself or to $-Y_{0}$, and one of which takes $Y_{0}$ to $-Y_{0}$, violating connectedness. The connected component of the compact matrix $\operatorname{group} \operatorname{Ad}(H)$ is its normal subgroup of the same dimension to which we apply the following facts to conclude the lemma.

Suppose that $d=2$, then $\tilde{\mathfrak{m}}$ is irreducible. If we identify $\tilde{\mathfrak{m}}$ with $\mathbb{R}^{2}$ then $H$ can be identified with $S O(2)$. Let $\left\{Y_{1}, Y_{2}\right\}$ be an orthonormal basis of $\mathfrak{m}$. Then

$$
\int_{H}\left\langle Y_{1}, \operatorname{Ad}(h) Y_{0}\right\rangle\left\langle Y_{2}, \operatorname{Ad}(h) Y_{0}\right\rangle d h=\int_{S O(2)}\left\langle Y_{1}, g Y_{0}\right\rangle\left\langle Y_{2}, g Y_{0}\right\rangle d g
$$

where $d g$ is the pushed forward measure $\operatorname{Ad}^{*}(d h)$. Since $d g$ is bi-invariant it is the standard measure on $S O(2)$, normalised to have volume 1 . Thus the above integral vanishes. This can be computed explicitly. The same proof as in Proposition 8.2 shows that $\int_{H}\left\langle Y, \operatorname{Ad}(h) Y_{0}\right\rangle^{2} d h=\left|Y_{0}\right|^{2} / 2$.

Proposition 8.2. Let $\tilde{\mathfrak{m}}$ be an $\operatorname{Ad}_{H}$ invariant subspace of $\mathfrak{m}$ not containing any non-trivial $\operatorname{Ad}_{H}$-invariant vectors. Suppose $Y_{0} \in \tilde{\mathfrak{m}}$ and let

$$
\overline{\mathcal{L}} f=\frac{1}{\lambda\left(Y_{0}\right)} \int_{H} \nabla^{L} d f\left(\left(\operatorname{Ad}(h)\left(Y_{0}\right)\right)^{*},\left(\operatorname{Ad}(h)\left(Y_{0}\right)\right)^{*}\right) d h .
$$

Then $\overline{\mathcal{L}}=\left(\left|Y_{0}\right|^{2} / \lambda\left(Y_{0}\right) \operatorname{dim}(\tilde{\mathfrak{m}})\right) \Delta_{\tilde{\mathfrak{m}}}$ under one of the following conditions:

(a) $\operatorname{dim}(\tilde{\mathfrak{m}})=2$; 
(b) $\operatorname{Ad}_{H}$ acts transitively and quasi doubly transitively on the unit sphere of $\tilde{\mathfrak{m}}$.

Proof. Expanding $\left(\operatorname{Ad}(h)\left(Y_{0}\right)\right)^{*}$ in $\left\{Y_{j}^{*}\right\}$ we see that $\overline{\mathcal{L}} f=\sum_{i, j=1}^{d} a_{i, j}\left(Y_{0}\right) \nabla^{L}$ $d f\left(Y_{i}^{*}, Y_{j}^{*}\right)$ where $a_{i, j}\left(Y_{0}\right)=\left(1 / \lambda\left(Y_{0}\right)\right) \int_{H}\left\langle Y_{i}, \operatorname{Ad}(h) Y_{0}\right\rangle\left\langle Y_{j}, \operatorname{Ad}(h)\left(Y_{0}\right)\right\rangle d h$. If $\operatorname{dim}(\tilde{\mathfrak{m}})=2$ then $\operatorname{Ad}(H)$ can be identified with $S O(2)$. In both cases, by Lemma 8.1, $a_{11}\left(Y_{0}\right)=\cdots=$ $a_{d d}\left(Y_{0}\right)$ and the cross terms disappear.

Since $\operatorname{ad}(\mathfrak{h})$ consists of skew symmetric matrices, with respect to the invariant Riemannian metric on the homogeneous manifold $G / H, H$ acts as a group of isometries. So do the left actions by elements of $H$ on $\pi_{*}(\tilde{\mathfrak{m}})$. Since $\pi$ takes the identity to the coset $H$, we may rule out translations and every element of $\operatorname{Ad}(h)$ is rotation on $\tilde{\mathfrak{m}}$. We identify $\tilde{\mathfrak{m}}$ with $\mathbb{R}^{d}$, where $d$ is the dimension of $\tilde{\mathfrak{m}}$. Since $H$ is connected, they are orientation preserving rotations. Since $H$ is compact, so is $\operatorname{Ad}(H)$ and $\operatorname{Ad}(H)$ can be identified with a compact subgroup of $S O(d)$.

It would be nice to classify subgroups of $S O(d)$ that acts transitively and quasi doubly transitively on the spheres. Sub-groups of $S O(d)$ acting transitively on the spheres are completely classified and coincide with the list of possible holonomy groups of simply connected, see [4], non-symmetric irreducible complete Riemannian manifolds [65] by Simons. See also Heintze and Ziller [29]. The large subgroups of $S O(d)$ are reasonably well understood, which is due to a theorem of Montgomery and Samelson [54] and also a theorem of Obata [58]. There are however two exceptions: the non-simple group $S O(4)$ and also $S O(8)$. The latter has two interesting subgroups: the 21 dimensional $\operatorname{spin}(7)$ and the exceptional 14 dimensional compact simple Lie group $G_{2}$, automorphism of the Octonians. We end this discussion with the following remark.

REMARK 8.3. Let $d=\operatorname{dim}(\tilde{\mathfrak{m}})$ where $\tilde{\mathfrak{m}}$ is $\operatorname{Ad}_{H}$-invariant. Suppose that $\operatorname{dim}\left(\left.\operatorname{Ad}(H)\right|_{\tilde{\mathfrak{m}}}\right) \geq \operatorname{dim}(S O(d-1))$; or suppose that $\tilde{\mathfrak{m}}$ has no two dimensional invariant subspace with $d \geq 13$ and suppose that $\operatorname{dim}\left(\left.\operatorname{Ad}(H)\right|_{\tilde{\mathfrak{m}}}\right) \geq \operatorname{dim}(O(d-3))+\operatorname{dim}(O(3))+1$. Then the identity component of $\operatorname{Ad}(H)_{\tilde{\mathfrak{m}}}$ is

1. $S O(d)$ if $d \neq 4,8$;

2. $S O(4)$ or $S^{3}$ if $d=4$;

3. $S O(8)$ or $\operatorname{spin}(7)$, if $d=8$.

Furthermore, $\operatorname{Ad}_{H}: H \rightarrow \mathbb{L}(\tilde{\mathfrak{m}} ; \tilde{\mathfrak{m}})$ acts transitively on the unit sphere of $\tilde{\mathfrak{m}}$, and acts quasi doubly transitively on $\tilde{\mathfrak{m}}$ if $d>2$.

Our aim is to prove that $\operatorname{Ad}_{H}$ acts transitively and quasi doubly transitively, using properties of connected closed subgroups of the rotation groups, we are not concerned with whether the image of $H$ under the representation, considered as subgroups of $S O(d)$, $d=\operatorname{dim}\left(\mathfrak{m}_{l}\right)$, is connected. It is sufficient to prove its identity component has the required property. The connected component of the compact matrix group $\operatorname{Ad}(H)$ is its normal subgroup of the same dimension to which we apply the following facts to conclude the lemma. For our purpose, the image of $\operatorname{Ad}_{H}$ acts faithfully on $\mathfrak{m}_{l}$ in the sense that if $H_{0}$ is the group of identity transformations, acting on $\mathfrak{m}_{l}$, then $H / H_{0}$ has the same orbit as $H_{0}$ and both act transitively. 
We have already seen that no $\mathrm{Ad}_{H}$-invariant subspace of $\mathfrak{m}$ can be one dimensional. By a theorem of Montgomery and Samelson [54, Lemma 3, 4], there is no proper closed subgroup $H^{\prime}$ of $S O(d)$ of dimension greater than the dimension of $S O(d-1)$. If $H^{\prime}$ is a connected closed sub-group of $S O(d)$ of the dimension of $S O(d-1)$, then $H$ is continuously isomorphic to $S O(d-1)$ or to the double cover of $S O(d-1)$. If $d \neq 4,8$, then $H$ is conjugate with $Q(d-1)$, the sub-matrix of $S O(d)$ leaving invariant the first axis. Note that the identity component of $\operatorname{Ad}(H)$ has the dimension of $\operatorname{Ad}(H)$. Since $\mathfrak{m}$ has no one dimensional invariant subspace we conclude that $\operatorname{Ad}(H)=S O(d)$ for $d=4,8$.

In dimension 4, the subgroup $S^{3}$ is not conjugate with $Q(d-1)$. The subgroup $S^{3}$ acts on itself transiently, freely, and leaves no one dimensional sub-space invariant. It is also doubly transitive.

If $d=8$, the 21-dimensional sub-group $\operatorname{spin}(7)$ is embedded into $S O(8)$ by the spin representation, it acts transitively on $S^{7}$ and its isotropy subgroup at a point is $G_{2}$. We learnt from Dmitriy Rumynin that $G_{2} \sim S U(4) \subset \operatorname{spin}(7)$ and $G_{2}$ act transitively on unit spheres of $S^{7}$ and transitive on any two pairs of orthogonal unit vectors. Finally we quote a theorem from Obata [58] : if $K$ is a Lie group of orthogonal $d \times d$ matrices where $d \geq 13$ and if $\operatorname{dim}(O(d))>\operatorname{dim}(K) \geq \operatorname{dim}(O(d-3))+\operatorname{dim}(d(3))+1$, then $K$ is reducible in the real vector space. Since $\mathfrak{m}_{l}$ is irreducible, the group $\operatorname{Ad}(H)$ must be $S O(d)$ where $d=\operatorname{dim}\left(m_{l}\right)$, and so the analysis on $S O(d)$ can be applied.

\section{Laplacian like operators as effective limits.}

Let $\left\{Y_{j}\right\}$ be an orthonormal basis of $\tilde{\mathfrak{m}}$. Denote $\Delta_{\tilde{\mathfrak{m}}}=\sum_{j=1}^{m} L_{Y_{j}^{*}} L_{Y_{j}^{*}}$, this is the 'round' operator on $\tilde{\mathfrak{m}}$. For the left invariant connection $\nabla^{L}, \Delta_{\tilde{\mathfrak{m}}}=\operatorname{trace}_{\mathfrak{m}} \nabla^{L} d$ is a 'generalised' horizontal Laplacian and is independent of the choice of the basis. In the special case where $G$ is isomorphic to the Cartesian product of a compact group and an additive vector group, there is a bi-invariant metric, $\nabla$ is the Levi-Civita connection for a bi-invariant metric, then $\nabla_{X} X^{*}=0$ and $\Delta_{\tilde{\mathfrak{m}}}=$ trace $_{\mathfrak{\mathfrak { m }}} \nabla d$. In the irreducible case, this operator $\Delta_{\mathfrak{m}}$ is the horizontal Laplacian and we denote the operator by $\Delta^{\text {hor }}$. Its corresponding diffusion is a horizontal Brownian motion. In the reducible case, we abuse the notation and define a similar concept. Since the limit operator in Theorem 6.10 is given by averaging the action of $\mathrm{Ad}_{H}$, we expect that the size of the isotropy group $H$ is correlated with the 'homogeneity' of the diffusion operator, which we explore in the remaining of the section.

Definition 9.1. A sample continuous Markov process is a (generalised) horizontal Brownian motion if its Markov generator is $(1 / 2) \Delta_{\tilde{\mathfrak{m}}}$; it is a (generalised) scaled horizontal Brownian motion with scale $c$ if its Markov generator is $(1 / 2) c \Delta_{\tilde{\mathfrak{m}}}$ for some constant $c \neq 0$.

We use the notation in Theorem 6.10. Let, $\alpha\left(Y_{j}, Y_{0}\right)=\left\langle Y_{j}, \operatorname{Ad}(h)\left(Y_{0}\right)\right\rangle$ and

$$
\overline{\mathcal{L}}=\sum_{i, j=1}^{d} a_{i, j}\left(Y_{0}\right) L_{Y_{i}^{*}} L_{Y_{j}^{*}}, \quad a_{i, j}\left(Y_{0}\right)=-\int_{H} \alpha\left(Y_{0}, Y_{i}\right) \mathcal{L}_{0}^{-1}\left(\alpha\left(Y_{0}, Y_{j}\right)\right) d h .
$$

If the representation space of $\operatorname{Ad}_{H}$ were complex, then $\left\langle Y, \operatorname{Ad}(h) Y_{0}\right\rangle$ where $Y, Y_{0} \in \mathfrak{m}_{l}$ 
are known as trigonometric functions.

TheOREM 9.1. Suppose that $\left\{A_{1}, \ldots, A_{N}\right\}$ generates $\mathfrak{h}, \mathcal{L}_{0}=(1 / 2) \sum_{k=1}^{N}\left(A_{k}\right)^{2}$, and $Y_{0} \in \mathfrak{m}_{l}$ where $l \neq 0$. If $Y_{0}=\sum_{m=1}^{d} c_{m} Y_{m}$, then

$$
\overline{\mathcal{L}}=\sum_{m=1}^{d} \frac{\left(c_{m}\right)^{2}}{\lambda\left(Y_{m}\right)} \frac{1}{\operatorname{dim}\left(\mathfrak{m}_{l}\right)} \Delta_{\mathfrak{m}_{l}}
$$

If furthermore $\left\{A_{1}, \ldots, A_{N}\right\}$ is an o.n.b. of $\mathfrak{h}$, then $\overline{\mathcal{L}}=\left(\left|Y_{0}\right|^{2} / \operatorname{dim}\left(\mathfrak{m}_{l}\right) \lambda_{l}\right) \Delta_{\mathfrak{m}_{l}}$.

Proof. Set $d=\operatorname{dim}\left(\mathfrak{m}_{l}\right)$. With respect to the $\operatorname{Ad}_{H}$-invariant inner product on $\mathfrak{g}, \operatorname{ad}^{2}\left(A_{k}\right)$ is a self-adjoint linear map on $\mathfrak{m}_{l}$. For an orthonormal basis $\left\{Y_{1}, \ldots, Y_{d}\right\}$ of $\mathfrak{m}_{l}$ consisting of eigenvectors of $(-1 / 2) \sum_{k=1}^{N} \operatorname{ad}^{2}\left(A_{k}\right)$ with $\lambda\left(Y_{j}\right)$ the corresponding eigenvalues. Then $\alpha\left(Y_{j}, Y_{0}\right)$ is an eigenfunction of $\mathcal{L}_{0}$ corresponding to the eigenvalue $-\lambda\left(Y_{0}\right)$, and $-\alpha\left(Y_{j}, Y_{0}\right) / \lambda\left(Y_{0}\right)$ solves the Poisson equation with right hand side $\alpha\left(Y_{j}, Y_{0}\right)$, see Lemma 7.2. Evidently $\lambda\left(Y_{j}\right) \neq 0$, for otherwise $\left\langle\sum_{k} \operatorname{ad}^{2}\left(A_{k}\right)\left(Y_{j}\right), Y_{j}\right\rangle=$ $-\sum_{k}\left|\operatorname{ad}\left(A_{k}\right)\left(Y_{j}\right)\right|^{2}=0$ which means $Y_{j}$ is in the kernel of $\operatorname{ad}_{H}$ which is possible only if $l=0$.

Consequently,

$$
\begin{aligned}
a_{i, j}\left(Y_{0}\right) & =-\sum_{m, m^{\prime}} c_{m} c_{m^{\prime}} \int_{H}\left\langle\operatorname{Ad}(h)\left(Y_{m^{\prime}}\right), Y_{i}\right\rangle \mathcal{L}_{0}^{-1}\left\langle\operatorname{Ad}(h)\left(Y_{m}\right), Y_{j}\right\rangle d h \\
& =\sum_{m, m^{\prime}} \frac{c_{m} c_{m^{\prime}}}{\lambda\left(Y_{m}\right)} \int_{H}\left\langle\operatorname{Ad}(h)\left(Y_{m^{\prime}}\right), Y_{i}\right\rangle\left\langle\operatorname{Ad}(h)\left(Y_{m}\right), Y_{j}\right\rangle d h .
\end{aligned}
$$

By Peter-Weyl's theorem, which states in particular that if $V$ is an irreducible unitary representation of a compact Lie group $H$ and $\left\{Y_{i}\right\}$ an o.n.b. of $V$, then the collection of functions $\left\{\left\langle Y_{i}, \rho(\cdot) Y_{k}\right\rangle\right\}$, where $V$ ranges through all equivalent classes of irreducible unitary representations $\rho$, is orthogonal with norm $\sqrt{\operatorname{dim}(V)}$, and these functions determine $L^{2}(G)$. In particular

$$
\int_{H}\left\langle Y_{i}, \operatorname{Ad}\left((h) Y_{k}\right)\right\rangle\left\langle Y_{j}, \operatorname{Ad}(h)\left(Y_{l}\right)\right\rangle d h=\frac{1}{d} \delta_{i j} \delta_{k l} .
$$

We are grateful to Dmitriy Rumynin for providing us with a version of this theorem, valid for orthogonal representations, which is appended at the end of the paper. See also Iwahori $[\mathbf{3 7}]$ on representations of real Lie algebras.

From this we see that $a_{i, j}=0$ for $i \neq j$ and

$$
a_{i, i}\left(Y_{0}\right)=\sum_{m=1}^{d} \frac{\left(c_{m}\right)^{2}}{\operatorname{dim}\left(\mathfrak{m}_{l}\right) \lambda\left(Y_{m}\right)}, \quad \overline{\mathcal{L}}=\frac{1}{d} \sum_{m=1}^{d} \frac{\left(c_{m}\right)^{2}}{\lambda\left(Y_{m}\right)} \sum_{i=1}^{m} L_{Y_{i}} L_{Y_{i}} .
$$

Then part (1) follows by setting

$$
\frac{1}{\lambda^{\prime}\left(Y_{0}\right)}=\sum_{m=1}^{d} \frac{\left(c_{m}\right)^{2}}{\lambda\left(Y_{m}\right)}
$$


Note that $\sum_{i=1}^{m} L_{Y_{i}} L_{Y_{i}}=$ trace $\nabla^{L} d$ is independent of the choice of the basis vectors $\left\{Y_{j}\right\}$ so is also $\mathcal{L}$, as a Markov generator to (4.4), which means $\lambda^{\prime}\left(Y_{0}\right)$ is independent of the choice of the basis vectors. For the case $\left\{A_{1}, \ldots, A_{N}\right\}$ is an orthonormal basis of $\mathfrak{h}$, we apply Lemma 7.1 to conclude.

If $Y_{0}$ belongs to a subspace of $\tilde{\mathfrak{m}}$, say $Y_{0} \in \mathfrak{m}_{l} \oplus \mathfrak{m}_{l^{\prime}}$, then an analogous claim holds if the representations $\operatorname{Ad}_{H}$ on $\mathfrak{m}_{l}$ and $\mathfrak{m}_{l^{\prime}}$ are not equivalent, especially if $\mathfrak{m}_{l}$ and $\mathfrak{m}_{l^{\prime}}$ have different dimensions.

Let $d=2$. Then $\left.\sum_{k} \operatorname{ad}^{2}\left(A_{k}\right)\right|_{\mathfrak{m}_{l}}=\lambda_{l}$ Id $\left.\right|_{\mathfrak{m}_{l}}$, for some number $\lambda_{l} \neq 0$, if and only if $\overline{\mathcal{L}}=\left(\left|Y_{0}\right|^{2} / 2 \lambda_{l}\right) \Delta_{\mathfrak{m}_{l}}$. Indeed, $H$ is essentially $S O(2)$. Let $Y_{1}, Y_{2}$ be a pair of orthogonal unit length eigenvectors of the linear map $(1 / 2) \sum_{k} \operatorname{ad}^{2}\left(A_{k}\right)$, restricted to $\mathfrak{m}_{l}$. Let $Y_{0}=c_{1} Y_{1}+c_{2} Y_{2}$. For $j^{\prime}, j, k^{\prime}, k=1,2$, let

$$
b_{j^{\prime}, j}^{k, k^{\prime}}\left(Y_{0}\right)=\frac{c_{k} c_{k^{\prime}}}{\lambda\left(Y_{k^{\prime}}\right)} \int_{S^{1}}\left\langle\operatorname{Ad}\left(e^{i 2 \pi \theta}\right)\left(Y_{k}\right), Y_{j^{\prime}}\right\rangle\left\langle\operatorname{Ad}\left(e^{i 2 \pi \theta}\right)\left(Y_{k^{\prime}}\right), Y_{j}\right\rangle d \theta .
$$

By direct computation, it is easy to see that $b_{j^{\prime}, j}^{k^{\prime}, k}=0$ unless $j^{\prime}=j, k=k^{\prime}$ or $j^{\prime}=k, j=$ $k^{\prime}$, In particular, we examine the cross term:

$$
\begin{aligned}
a_{1,2}\left(Y_{0}\right)= & \sum_{k, l=1}^{2} b_{1,2}^{k, l}=b_{1,2}^{2,1}+b_{1,2}^{1,2} \\
= & \frac{c_{1} c_{2}}{\lambda\left(Y_{2}\right)} \int_{S^{1}}\left\langle\operatorname{Ad}\left(e^{i 2 \pi \theta}\right)\left(Y_{1}\right), Y_{1}\right\rangle\left\langle\operatorname{Ad}\left(e^{i 2 \pi \theta}\right)\left(Y_{2}\right), Y_{2}\right\rangle d \theta \\
& +\frac{c_{1} c_{2}}{\lambda\left(Y_{1}\right)} \int_{S^{1}}\left\langle\operatorname{Ad}\left(e^{i 2 \pi \theta}\right)\left(Y_{2}\right), Y_{1}\right\rangle\left\langle\operatorname{Ad}\left(e^{i 2 \pi \theta}\right)\left(Y_{1}\right), Y_{2}\right\rangle d \theta .
\end{aligned}
$$

Direct computation shows that

$$
\begin{aligned}
a_{1,2}\left(Y_{0}\right) & =\frac{c_{1} c_{2}}{\lambda\left(Y_{2}\right)} \int \cos ^{2}(i 2 \pi \theta) d \theta-\frac{c_{1} c_{2}}{\lambda\left(Y_{1}\right)} \int \sin ^{2}(i 2 \pi \theta) d \theta \\
& =\frac{1}{2}\left(\frac{c_{1} c_{2}}{\lambda\left(Y_{2}\right)}-\frac{c_{1} c_{2}}{\lambda\left(Y_{1}\right)}\right) .
\end{aligned}
$$

Thus

$$
\overline{\mathcal{L}}=\left(\frac{c_{1} c_{2}}{\lambda\left(Y_{2}\right)}-\frac{c_{1} c_{2}}{\lambda\left(Y_{1}\right)}\right) L_{Y_{1}} L_{Y_{2}}+\frac{1}{2}\left(\frac{c_{1}^{2}}{\lambda\left(Y_{1}\right)}+\frac{c_{2}^{2}}{\lambda\left(Y_{2}\right)}\right) \Delta_{\mathfrak{m}_{l}} .
$$

Thus $a_{1,2}\left(Y_{0}\right)$ vanishes if and only if $\lambda_{l}:=\lambda\left(Y_{2}\right)=\lambda\left(Y_{1}\right)$, the latter allows us to conclude that $a_{1,1}\left(Y_{0}\right)=a_{2,2}\left(Y_{0}\right)=\left(\left|Y_{0}\right|^{2} / \lambda_{l}\right) / 2$ and $(1 / 2) \sum_{k} \operatorname{ad}^{2}\left(A_{k}\right)=\lambda_{l}$ Id.

We next work with semi-simple Lie groups. A Lie algebra is simple if it is not one dimensional and if $\{0\}$ and $\mathfrak{g}$ are its only ideals; it is semi-simple if it is the direct sum of simple algebras. Cartans criterion for semi-simplicity states that $\mathfrak{g}$ is semi-simple if and only if its killing form is non-degenerate. Another useful criterion is that a lie algebra is semi-simple if and only if it has no solvable (i.e. Abelian) ideals. The special unitary group $S U(n)$ is semi-simple if $n \geq 2 ; S O(n)$ is semi-simple for $n \geq 3 ; S L(n, \mathbb{R})$ is a non-compact semi-simple Lie group for $n \geq 2 ; \mathfrak{s o}(p, q)$ is semi-simple for $p+q \geq 3$. 
Corollary 9.2. Let $H$ be a maximal torus group of a semi-simple group $G$. Let $Y_{0} \in \mathfrak{m}_{l}$ and suppose that $A_{0}=0$ and $\left\{A_{i}\right\}$ generates $\mathfrak{h}$. Then $\overline{\mathcal{L}}=\left(\left|Y_{0}\right|^{2} / 2 \lambda_{l}\right) \Delta_{\mathfrak{m}_{l}}$ where $\lambda_{l}$ is determined by $(1 / 2) \sum_{k=1}^{p} \operatorname{ad}^{2}\left(A_{k}\right)=-\lambda_{l} \operatorname{Id}_{\mathfrak{m}_{l}}$.

Proof. If $\mathfrak{h}$ is the Cartan sub-algebra of the semi-simple Lie algebra $\mathfrak{g}$ the dimension of $\mathfrak{m}_{l}$ is 2 and Proposition 8.2 applies. It is clear that $\operatorname{dim}\left(\mathfrak{m}_{l}\right) \geq 2$, for otherwise it consists of invariant vectors. It is well known that $\mathfrak{g}$ is semi-simple if and only if $\mathfrak{g}_{\mathbb{C}}=\mathfrak{g} \otimes \mathbb{C}$ is semi-simple. Denote the complexification of $\mathfrak{h}$ by $\mathfrak{t}$, it is a Cartan subalgebra of $\mathfrak{g} \otimes \mathbb{C}$. Let $\alpha \in \mathfrak{t}^{*}$ be a weight for $\mathfrak{g}_{\mathbb{C}}$. Take $Y=Y_{1}+i Y_{2}$ from the root space $\mathfrak{g}_{\alpha}$ corresponding to $\alpha$. Let $X \in \mathfrak{h}$, and write $\alpha=\alpha_{1}+i \alpha_{2}$. Then

$$
[X, Y]=\left[X, Y_{1}\right]+i\left[X, Y_{2}\right]=\left(\alpha_{1}(X)+i \alpha_{2}(X)\right)\left(Y_{1}+i Y_{2}\right)
$$

and $Y_{1}$ and $Y_{2}$ generate an invariant subspace for $\operatorname{ad}_{H}$ following from:

$$
\left[X, Y_{1}\right]=\alpha_{1}(X) Y_{1}-\alpha_{2}(X) Y_{2} ; \quad\left[X, Y_{2}\right]=\alpha_{1}(X) Y_{1}+\alpha_{2}(X) Y_{2}
$$

Restricted on each vector space $\mathfrak{g}_{\alpha}$ only one specific $A_{k}$ in the sum $\sum_{k=1}^{p} \operatorname{ad}^{2}\left(A_{k}\right)$ makes non-trivial contribution to the corresponding linear operation. Thus we can restrict to the one dimensional torus sub-group of $H$, which acts faithfully on $\mathfrak{g}_{\alpha}$. By the earlier discussion, $\overline{\mathcal{L}}=\left(\left|Y_{0}\right|^{2} / \lambda_{l} \operatorname{dim}\left(\mathfrak{m}_{l}\right)\right) \Delta_{\mathfrak{m}_{l}}=\left(1 / 2 \lambda_{l}\right)\left|Y_{0}\right|^{2} \Delta_{\mathfrak{m}_{l}}$.

EXAMPLE 9.3. Let $G_{0}(k, n)=S O(n) / S O(k) \times S O(n-k)$ be the oriented Grassmannian manifold of $k$ oriented planes in $n$ dimensions. It is a connected manifold of dimension $k(n-k)$. The Lie group $S O(n)$ act on it transitively. Let $o$ be $k$-planes spanned by the first $k$ vectors of the standard basis in $\mathbb{R}^{n}$. Then $S O(k) \times O(n-k)$ keeps $\mathbb{R}^{k}$ fixed and as well as keeps the orientation of the first $k$-frame. Let $\pi: S O(n) \rightarrow G(k, n)$, then $\pi O=\left\{O_{1}, \ldots, O_{k}\right\}$, the first $k$ columns of the matrix $O$. Let $\sigma(A)=S A S^{-1}$, where $S$ is the diagonal block matrix with $-I_{k}$ and $I_{n-k}$ as entries, be the symmetry map on $G$. The Lie algebra has the symmetric decomposition $\mathfrak{s o}(n)=\mathfrak{h} \oplus \mathfrak{m}$ :

$$
\mathfrak{h}=\left\{\left(\begin{array}{cc}
\mathfrak{s o}(k) & 0 \\
0 & \mathfrak{s o}(n-k)
\end{array}\right)\right\}, \quad \mathfrak{m}=\left\{Y_{M}:=\left(\begin{array}{cc}
0 & M \\
-M^{T} & 0
\end{array}\right), M \in M_{k, n-k}\right\} .
$$

The adjoint action on $\mathfrak{m}$ is given by

$$
\operatorname{Ad}(h)\left(Y_{M}\right)=\left(\begin{array}{cc}
0 & R M Q^{T} \\
-\left(R M Q^{T}\right)^{T} & 0
\end{array}\right)
$$

for $h=\left(\begin{array}{ll}R & 0 \\ 0 & Q\end{array}\right), R \in S O(k)$ and $Q \in S O(n-k)$. We identify $\mathfrak{m}$ with $T_{o} G_{0}(k, n)$. The isotropy action on $T_{o} G_{0}(k, n)$ is now identified with the map $\left(\left(\begin{array}{ll}R & 0 \\ 0 & Q\end{array}\right), M\right) \rightarrow R M Q^{T}$. Let $M_{1}=\left(e_{1}, 0, \ldots, 0\right)$. Then $R M_{1} Q^{T}=R_{1} Q_{1}^{T}$ where $R_{1}$ and $Q_{1}$ are the first columns of $R$ and $Q$ respectively. The orbit of $M_{1}$ by the Adjoint action generates a basis of $M_{k, n-k}$ and $M$ is isotropy irreducible. Let $M_{2}=\left(0, e_{2}, 0, \ldots, 0\right), d R$ and $d Q$ the Haar measure on $S O(k)$ and $S O(n-k)$ respectively. Then if $R=\left(r_{i j}\right)$ and $Q=\left(q_{i j}\right)$. Then 


$$
\int_{H}\left\langle\operatorname{Ad}(h) M_{1}, M_{1}\right\rangle\left\langle\operatorname{Ad}(h)\left(M_{1}\right), M_{2}\right\rangle d R d Q=\int_{H}\left(r_{11}\right)^{2} q_{11} q_{12} d Q d R=0 .
$$

Hence $\overline{\mathcal{L}}$ is proportional to $\Delta^{\text {hor }}$. It is easy to see that $\overline{\mathcal{L}}$ satisfies the one step Hörmander condition and is hypoelliptic on $G$. Given $M, N \in M_{k \times(n-k)}$ whose corresponding elements in $\mathfrak{m}$ denoted by $\tilde{M}, \tilde{N}$. Then $[\tilde{M}, \tilde{N}]=N^{T} M-M N^{T}$. There is a basis of $\mathfrak{h}$ in this form. If $\left\{e_{i}\right\}$ is the standard basis of $\mathbb{R}^{k}, E_{i j}=e_{i} e_{j}^{T},\left\{E_{i j}-E_{j i}, i<j\right\}$ is a basis of $\mathfrak{s o}(\mathfrak{k})$.

\section{Classification of limits on Riemannian homogeneous manifolds.}

Let $M$ be a smooth manifold with a transitive action by a Lie group $G$. A Riemannian metric on $M$ is $G$-invariant if $\bar{L}_{a}$ for all $a \in G$, are isometries, in which case $M$ is a Riemannian homogeneous space and $G$ is a subgroup of $\operatorname{Iso}(M)$. We identify $G$ with the group of actions and $M$ with $G / H$ where $H=G_{o}$, the subgroup fixing a point $o$. By declaring $d \pi$ at the identity an isometry, an $\operatorname{Ad}(H)$-invariant inner product on $\mathfrak{m}$ induces a $G$ invariant inner product on $T_{o} M$ and vice versa. This extends to a $G$ invariant Riemannian metric by defining: $\left\langle\left(d \bar{L}_{g}\right)_{o} \pi_{*} Y_{1},\left(d \bar{L}_{g}\right)_{o} \pi_{*} Y_{2}\right\rangle_{g o}=\left\langle\pi_{*} Y_{1}, \pi_{*} Y_{2}\right\rangle_{o}$. Furthermore, $G$-invariant metrics on $M$ are in one to one correspondence with $\operatorname{Ad}_{H}$-invariant metrics on $\mathfrak{m}$. We should mention that, by a theorem of Myers and Steenrod [55], the set of all isometries of a Riemannian manifold $M$ is a Lie group under composition of maps, and furthermore the isotropy subgroup $\operatorname{Iso}_{o}(M)$ is compact. See also Kobayashi and Nomizu [40]. If a subgroup $G$ of $\operatorname{Iso}(M)$ acts on $M$ transitively, $G / H$ is a Riemannian homogeneous space, in the sense that $G$ acts effectively on $M$.

A connected Lie group admits an Ad-invariant metric if and only if $G$ is of compact type, i.e. $G$ is isomorphic to the Cartesian product of a compact group and an additive vector group [53, Lemma 7.5], in which case we choose to use the bi-invariant metric for simplicity. The existence of an $\mathrm{Ad}_{H}$-invariant metric is less restrictive. If $H$ is compact by averaging we can construct an $\mathrm{Ad}_{H}$ invariant inner product in each irreducible invariant subspace of $\mathfrak{m}$. If $\mathfrak{m}=\mathfrak{m}_{0} \oplus \bigoplus_{i=1}^{r} \mathfrak{m}_{i}$ is an irreducible invariant decomposition of $\mathfrak{m}$, $\mathrm{Ad}_{H}$-invariant inner products on $\mathfrak{m}$ are precisely of the form $g_{0}+\sum_{i=1}^{r} a_{i} g_{i}$ where $g_{0}$ is any inner product on $\mathfrak{m}_{0}, a_{i}$ are positive numbers and $g_{i}$ are $\operatorname{Ad}_{H}$ invariant inner products on $\mathfrak{m}_{i}$. In particular an irreducible homogeneous space with compact $H$ admits a $G$-invariant Riemannian metric, unique up to homotheties. See Wolf [69] and Besse [7, Theorem 7.44].

In the remaining of the section we assume that $G$ is given a left invariant Riemannian metric which induces a $G$-invariant Riemannian metric on $M$ as constructed. We ask the question whether the projection of the limiting stochastic process in Theorem 7.3 is a Brownian motion like process. Firstly, we note that the projections of 'exponentials', $g \exp (t X)$, are not necessarily Riemannian geodesics on $M$. They are not necessarily Riemannian exponential maps on $G$. Also, given an orthonormal basis $\left\{Y_{i}, 1 \leq i \leq n\right\}$ of $\mathfrak{g}, \sum_{i=1}^{n} L_{Y_{i}^{*}} L_{Y_{i}^{*}}$ is not necessarily the Laplace-Beltrami operator.

A connected Lie group $G$ admits a bi-invariant Riemannian metric if and only if its Lie algebra admits an $\operatorname{Ad}_{G}$ invariant inner product, the latter is equivalent to $\operatorname{ad}(X)$ is skew symmetric for every $X \in \mathfrak{g}$. It is also equivalent to that $G$ is the Cartesian product 
of a compact group and an additive vector space. For $X, Y, Z \in \mathfrak{g}$, Koszul's formula for the Levi-Civita connection of the left-invariant metric on $G$ gives:

$$
\left\langle 2 \nabla_{X} Y, Z\right\rangle=\langle[X, Y], Z\rangle+\langle[Z, Y], X\rangle+\langle[Z, X], Y\rangle .
$$

By polarisation, $\nabla_{X} X=0$ for all $X$ if and only if

$$
\langle[Z, Y], X\rangle+\langle[Z, X], Y\rangle=0, \quad \forall X, Y, Z,
$$

in which case $\nabla_{X} Y=(1 / 2)[X, Y]$. In another word, (10.1) holds if and only if $\nabla^{L}$ and $\nabla$ have the same set of geodesics, they are translates of the one parameter subgroups. If the Riemannian metric on $G$ is bi-invariant then translates of the one parameter subgroups are indeed geodesics for the Levi-Civita connection and for the family of connections interpolating left and right invariant connections. A connection is torsion skew symmetric if its torsion $\mathcal{T}$ satisfies: $\langle\mathcal{T}(u, v), w\rangle+\langle\mathcal{T}(u, w), v\rangle=0$ for all $u, v, w \in T_{x} M$ and $x \in M$. Denote by $T^{L}(X, Y)$ the torsion for the flat connection $\nabla^{L}$. Since $T^{L}(X, Y)=-[X, Y]$, (10.1) is equivalent to $\nabla^{L}$ being torsion skew symmetric. By Milnor, if a connected Lie group has a left invariant connection whose Ricci curvatures is non-negative then $G$ is unimodular. We expand this in the following lemma, the unimodular case is essentially Lemma 6.3 in Milnor [53].

Lemma 10.1. Let $G$ be a connected Lie group with a left invariant metric. Then $\sum_{i=1}^{n} L_{Y_{i}^{*}} L_{Y_{i}^{*}}=\Delta$ if and only if $G$ is unimodular. If $\mathfrak{m}_{l}$ is a subspace of $\mathfrak{g}$, then trace $_{\mathfrak{m}_{l}} \operatorname{ad}(X)=0$ for all $X \in \mathfrak{g}$ if and only if $\operatorname{trace}_{\mathfrak{m}_{l}} \nabla d=$ trace $_{\mathfrak{m}_{l}} \nabla^{L} d$.

Proof. A Lie group $G$ is unimodular if its left invariant Haar measure is also right invariant. By Helgson [30], a Lie group is unimodular if and only if the absolute value of the determinant of $\operatorname{Ad}(g): G \rightarrow G$ is 1 for every $g \in G$. Equivalently ad(X) has zero trace for every $X \in \mathfrak{g}$, see [53, Lemma 6.3]. On the other hand $\sum_{i=1}^{n} L_{Y_{i}^{*}} L_{Y_{i}^{*}}=\Delta$ if and only if $\sum_{i=1}^{n} \nabla_{Y_{i}{ }^{*}} Y_{i}^{*}=0$. The latter condition is equivalent to $\operatorname{trace} \operatorname{ad}(X)=\sum_{i}\left\langle\left[X, Y_{i}\right], Y_{i}\right\rangle=0$ for all $X \in \mathfrak{g}$. Similarly if $\left\{Y_{i}\right\}$ is an orthonormal basis of $\mathfrak{m}_{l}$, then

$$
\sum_{i=1}^{\operatorname{dim}\left(\mathfrak{m}_{l}\right)} L_{Y_{i}^{*}} L_{Y_{i}^{*}}=\sum_{i=1}^{\operatorname{dim}\left(\mathfrak{m}_{l}\right)} \nabla d+\sum_{i=1}^{\operatorname{dim}\left(\mathfrak{m}_{l}\right)} \nabla_{Y_{i}^{*}} Y_{i}^{*}
$$

The second statement follows from the identity:

$$
\left\langle\sum_{i=1}^{\operatorname{dim}\left(\mathfrak{m}_{l}\right)} \nabla_{Y_{i}^{*}} Y_{i}^{*}, X^{*}\right\rangle=\sum_{i=1}^{\operatorname{dim}\left(\mathfrak{m}_{l}\right)}\left\langle\left[X, Y_{i}\right], Y_{i}\right\rangle .
$$

We discuss the relation between the Levi-Civita connection and the canonical connection on the Riemannian homogeneous manifold. A connection is $G$ invariant if $\left\{\bar{L}_{a}, a \in G\right\}$ are affine maps for the connection, so they preserve parallel vector fields. For $X \in \mathfrak{g}$, define the derivation $A_{X}=L_{X}-\nabla_{X}$. A $G$-invariant connection is determined by a linear map $\left(A_{X}\right)_{o} \in \mathbb{L}\left(T_{o} M ; T_{o} M\right)$. Each $A_{X}$ is in correspondence with an endomorphism $\Lambda_{\mathfrak{m}}(X)$ on $\mathbb{R}^{n}$, satisfying the condition that for all $X \in \mathfrak{m}$ and $h \in H$, 
$\Lambda_{\mathfrak{m}}(\operatorname{ad}(h) X)=\operatorname{Ad}(\lambda(h)) \Lambda_{\mathfrak{m}}(X)$ where $\lambda(h)$ is the isotropy representation of $h$, see Kobayashi and Nomizu [40]. Then

$$
\tilde{\nabla}_{X} Y:=L_{X} Y-u_{0} \Lambda_{\mathfrak{m}}(X) u_{0}^{-1} Y
$$

defines a connection. We identified $T_{o} M$ with $\mathbb{R}^{n}$ by a frame $u_{0}$. If $\Lambda_{\mathfrak{m}}=0$ this defines the canonical connection $\nabla^{c}$ whose parallel translation along a curve is left translation.

Denote by $D^{c} / d t$ the corresponding covariant differentiation. Parallel translations along $\alpha(t)$ are given by $d \bar{L}_{\gamma(t)}$. This is due to the fact that left translations and $\pi$ commute. If $X \in \mathfrak{m}$, let $\gamma_{t}=\gamma_{0} \exp (t X)$ and $\alpha(t)=\gamma_{0} \exp (t X) o$. Then $\gamma$ is the horizontal lift of $\alpha$ and $\alpha(t)$ is a $\nabla^{c}$ geodesic,

$$
\frac{D^{c}}{d t} \dot{\alpha}=\frac{D^{c}}{d t} \pi_{*} T L_{\gamma(t)}(X)=\frac{D^{c}}{d t} T \bar{L}_{\gamma(t)} \pi_{*}(X)=0 .
$$

For $x \in \mathfrak{g}$, denote by $X_{m}$ and $X_{\mathfrak{h}}$ the component of $X$ in $\mathfrak{m}$ and in $\mathfrak{h}$ respectively.

DEFINITION 10.1. A reductive Riemannian homogeneous space is naturally reductive, if

$$
\left\langle[X, Y]_{\mathfrak{m}}, Z\right\rangle=-\left\langle Y,[X, Z]_{\mathfrak{m}}\right\rangle, \quad \text { for all } X, Y, Z \in \mathfrak{m} .
$$

If $G$ is of compact type, then $G / H$ is reductive with reductive structure $m=\mathfrak{h}^{\perp}$ and is naturally reductive, with respect to the bi-invariant metric.

It is clear that (10.1) implies (10.2) and $M$ is naturally reductive if $\nabla$ and $\nabla^{L}$ have the same set of geodesics. In particular, if $G$ admits an $\operatorname{ad}_{G}$ invariant inner product and $\mathfrak{m}=\mathfrak{h}^{\perp}$, then $G / H$ is naturally reductive with respect to the induced Riemannian metric. A special reductive homogeneous space is a symmetric space with symmetry $\sigma$ and the canonical decomposition $\mathfrak{g}=\mathfrak{h} \oplus \mathfrak{m}$. For it, $[\mathfrak{h}, \mathfrak{m}] \subset \mathfrak{m}$ and $[\mathfrak{m}, \mathfrak{m}] \subset \mathfrak{h}$. If the symmetric decomposition is orthogonal, it is naturally reductive.

We collect in the lemma below useful information for the computations of the Markov generators whose proof is included for the convenience of the reader.

LEMMA 10.2. $\quad 1 . \nabla^{c}$ is torsion skew symmetric precisely if $M$ is naturally reductive.

2. The projections of the translates of one parameter family of subgroups of $G$ are geodesics for the Levi-Civita connection if and only if $M$ is naturally reductive.

3. Let $U: \mathfrak{m} \times \mathfrak{m} \rightarrow \mathfrak{m}$ be defined by

$$
2\langle U(X, Y), Z\rangle=\left\langle X,[Z, Y]_{\mathfrak{m}}\right\rangle+\left\langle[Z, X]_{\mathfrak{m}}, Y\right\rangle .
$$

Then trace $_{\mathfrak{m}_{l}} \nabla^{c} d f=$ trace $_{\mathfrak{m}_{l}} \nabla d f$ if and only if trace $\mathfrak{m}_{l} U=0$. In other words, for any $Z \in \mathfrak{m}, \operatorname{trace}_{\mathfrak{m}_{l}} \operatorname{ad}(Z)=0$. In particular $\operatorname{trace}_{\mathfrak{m}_{l}} \nabla^{c} d f=\operatorname{trace}_{\mathfrak{m}_{l}} \nabla d f$, if $M$ is naturally reductive.

4. $\nabla^{c} d \pi=0$. 
Proof. (1) Let $Y^{*}$ denote the action field generated by $Y \in \mathfrak{m}$, identified with $\pi_{*}(Y) \in T_{o} M$, and $Y^{*}(u o)=\left.(d / d t)\right|_{t=0} \bar{L}_{\exp (t Y)} u o=\left.(d / d t)\right|_{t=0} \pi(\exp (t Y) u)$. If $p=u o$, $\nabla_{X}^{c} Y^{*}(o)=\left[X^{*}, Y^{*}\right](o)=-[X, Y]_{\mathfrak{m}}$. The torsion tensor for $\nabla^{c}$ is left invariant, its value at $o$ is

$$
\mathcal{T}_{o}^{c}(X, Y)=\left(\nabla_{X}^{c} Y^{*}\right)_{o}-\left(\nabla_{Y}^{c} X^{*}\right)_{o}-\left[X^{*}, Y^{*}\right]_{o}=-[X, Y]_{\mathfrak{m}} .
$$

We see that $\mathcal{T}^{c}$ is skew symmetric if and only if (10.2) holds.

(2) A $G$-invariant connection on $M$ has the same set of geodesics as $\nabla^{c}$ if and only if $\Lambda_{\mathfrak{m}}(X)(X)=0$, c.f. Kobayashi and Nomizu [40]. It is well known that the function

$$
\Lambda_{\mathfrak{m}}(X)(Y)=\frac{1}{2}[X, Y]_{\mathfrak{m}}+U(X, Y)
$$

defines the Levi-Civita connection. In fact it is clear that $(1 / 2)[X, Y]-U(X, Y)$ has vanishing torsion and $\langle\Lambda(X, Y), Z\rangle+\langle Y, \Lambda(X, Z)\rangle=0$, which together with the fact $\nabla^{c}$ is metric implies that it is a Riemannian connection. The Levi-Civita connection $\nabla$ and $\nabla^{c}$ have the same set of geodesics if and only if $U(X, X)=0$. By polarisation, this is equivalent to $M$ being naturally reductive.

(3) For any $X, Y \in \mathfrak{m}$,

$$
\left(\nabla_{X} Y^{*}\right)_{o}=\nabla_{X}^{c} Y^{*}+\frac{1}{2}[X, Y]_{\mathfrak{m}}+U(X, Y)
$$

If $f: M \rightarrow \mathbb{R}$ is a smooth function,

$$
\begin{aligned}
\left\langle\nabla_{X} \nabla f, Y\right\rangle & =L_{Y}\left(d f\left(Y^{*}\right)\right)-\left\langle\nabla f, \nabla_{X} Y^{*}\right\rangle \\
& =\left\langle\nabla_{X}^{c} \nabla f, Y\right\rangle+\left\langle\nabla f, \nabla_{X}^{c} Y^{*}-\nabla_{X} Y^{*}\right\rangle .
\end{aligned}
$$

Summing over the basis of $\mathfrak{m}_{l}$ we see that

$$
\operatorname{trace}_{\mathfrak{m}_{l}} \nabla d f-\operatorname{trace}_{\mathfrak{m}_{l}} \nabla^{c} d f=\left\langle\nabla f, \sum_{i} U\left(Y_{i}, Y_{i}\right)\right\rangle,
$$

which vanishes for all smooth $f$ if and only if $\sum_{i} U\left(Y_{i}, Y_{i}\right)$ vanishes. If $G$ is of compact type, it has an $\operatorname{Ad}_{G}$-invariant metric for which (10.1) holds. This completes the proof.

(4) We differentiate the map $d \pi: G \mapsto \mathbb{L}(T G ; T M)$ with respect to the connection $\nabla^{c}$. Let $\gamma(t)$ be a curve in $G$ with $\gamma(0)=u$ and $\dot{\gamma}(0)=w$. Then for $X \in \mathfrak{g}$,

$$
\left(\nabla_{w}^{c} d \pi\right)(X)=/ /_{t}^{c}(\pi \circ \gamma) \frac{d}{d t}\left(/ /_{t}^{c}(\pi \circ \gamma)\right)^{-1} d \pi\left(/ t_{t}^{L}(\gamma) X\right),
$$

where $/{ }_{t}^{c}(\pi \circ \gamma)$ and $/ /_{t}^{L}(\gamma)$ denote respectively parallel translations along $\pi \circ \gamma$ and $\gamma$ with respect to $\nabla^{c}$ and $\nabla^{L}$. Since $/ /_{t}^{c}(\pi \circ \gamma)=d \bar{L}_{\pi \circ \gamma(t)}$ and $/{ }_{t}^{L}(\gamma)=d L_{\gamma(t)}$ the covariant derivative vanishes. Indeed $\pi$ and left translation commutes, $(d \pi)_{u} d L_{u}=\left(d \bar{L}_{u}\right)_{o}(d \pi)_{1}$,

$$
\nabla_{w}^{c} d \pi=/\left.\left.\right|_{t} ^{c}(\pi \circ \gamma) \frac{d}{d t}\right|_{t=0}(d \pi)_{1}\left(d L_{\gamma(-t)} / /_{t}^{L}(\gamma)\right)=0
$$

In the propositions below we keep the notation in Theorem 6.10, Theorem 7.3 and 
Proposition 8.2. We identify $\mathfrak{m}_{l}$ with its projection to $T_{o} M$. Let $\bar{x}_{t}:=\lim _{\epsilon \rightarrow 0} x_{t / \epsilon}^{\epsilon}$.

Proposition 10.3. Suppose that $\overline{\mathcal{L}}=\left|Y_{0}\right|^{2} / \lambda\left(Y_{0}\right) \operatorname{dim}\left(\mathfrak{m}_{l}\right) \Delta_{\mathfrak{m}_{l}}$. If $\operatorname{trace}_{\mathfrak{m}_{l}} U=0$, equivalently $\operatorname{trace}_{\mathfrak{m}_{l}} \operatorname{ad}(Z)=0$ for all $Z \in \mathfrak{m}$, then $\left(\bar{x}_{t}\right)$ is a Markov process with generator

$$
\frac{\left|Y_{0}\right|^{2}}{\lambda\left(Y_{0}\right) \operatorname{dim}\left(\mathfrak{m}_{l}\right)} \operatorname{trace}_{\mathfrak{m}_{l}} \nabla d .
$$

If $M$ is furthermore isotropy irreducible, $\left(\bar{x}_{t}\right)$ is a scaled Brownian motion.

Proof. Let $\left\{\tilde{Y}_{i}, 1 \leq i \leq m_{l}\right\}$ be an orthonormal basis of $\mathfrak{m}_{l}$ and let $(1 / 2) a^{2}=$ $\left|Y_{0}\right|^{2} / \operatorname{dim}\left(\mathfrak{m}_{l}\right) \lambda_{l}$. Let $Y_{i}=a \tilde{Y}_{i}$. Let $\left(u_{t}\right)$ be a Markov process with generator $(1 / 2) \sum_{k=1}^{\operatorname{dim}\left(m_{l}\right)} L_{Y_{k}^{*}} L_{Y_{k}^{*}}$, and represented by a solution of the left invariant SDE: $d u_{t}=$ $\sum_{k=1}^{m_{l}} Y_{k}^{*}\left(u_{t}\right) \circ d B_{t}^{k}$ where $\left\{B_{t}^{k}\right\}$ are independent one dimensional Brownian motions. Let $y_{t}=\pi\left(u_{t}\right)$. Denote by $\phi_{t}^{k}$ the integral flow of $Y_{k}, \phi_{t}^{i}(g)=g \exp \left(t Y_{i}\right)$. If $f \in C_{K}^{2}(M ; \mathbb{R})$,

$$
\begin{aligned}
f \circ \pi\left(u_{t}\right)= & f \circ \pi\left(u_{0}\right)+\left.\sum_{i=1}^{m_{l}} \int_{0}^{t} \frac{d}{d t} f \circ \pi\left(u_{r} \exp \left(t Y_{i}\right)\right)\right|_{t=0} d B_{r}^{i} \\
& +\left.\frac{1}{2} \sum_{i=1}^{m_{l}} \int_{0}^{t} \frac{d^{2}}{d t^{2}} f \circ \pi\left(u_{r} \exp \left(t Y_{i}\right)\right)\right|_{t=0} d r .
\end{aligned}
$$

We compute the last term beginning with the first order derivative,

$$
\left.\frac{d}{d t} f \circ\left(u_{r} \exp \left(t X_{i}\right)\right)\right|_{t=0}=\left.d f\left(\pi_{*}\left(L_{u_{r} \exp \left(t Y_{i}\right)} Y_{i}\right)\right)\right|_{t=0}=d f\left(\left(\bar{L}_{u_{r}}\right)_{*} X_{i}\right) .
$$

Let $D / d t$ denote covariant differentiation along the curve $\left(x_{t}\right)$ with respect to the Levi-Civita connection.

$$
\begin{aligned}
& \left.\sum_{i=1}^{m_{l}} \frac{d^{2}}{d t^{2}} f \circ \pi\left(u_{r} \exp \left(t Y_{i}\right)\right)\right|_{t=0} \\
& =\left.\sum_{i=1}^{m_{l}} \frac{d}{d t} d f\left(\left(\bar{L}_{u_{r} \exp \left(t Y_{i}\right)}\right)_{*}\left(\pi_{*}\left(Y_{i}\right)\right)\right)\right|_{t=0} \\
& =\sum_{i=1}^{m_{l}} \nabla d f\left(\left(\bar{L}_{u_{r}}\right)_{*}\left(\pi_{*}\left(Y_{i}\right)\right),\left(\bar{L}_{u_{r}}\right)_{*}\left(\pi_{*}\left(Y_{i}\right)\right)\right)+\sum_{i=1}^{m_{l}} d f\left(\left.\frac{D}{d t}\left(\bar{L}_{u_{r} \exp \left(t Y_{i}\right)}\right)_{*}\left(\pi_{*}\left(Y_{i}\right)\right)\right|_{t=0}\right) .
\end{aligned}
$$

Since left translations are isometries, for each $u \in G,\left\{\left(\bar{L}_{u}\right)_{*}\left(\pi_{*}\left(Y_{i}\right)\right)\right\}$ is an orthonormal basis of $\pi_{*}\left(u \mathfrak{m}_{l}\right) \subset T_{\pi(u)} M$. Thus

$$
\left.\sum_{i=1}^{m_{l}} \frac{d^{2}}{d t^{2}} f \circ \pi\left(u_{r} \exp \left(t Y_{i}\right)\right)\right|_{t=0}=a^{2} \operatorname{trace}_{\mathfrak{m}_{l}} \nabla^{c} d f+\sum_{i=1}^{\mathfrak{m}_{l}} d f\left(\left.\frac{D^{c}}{d t}\left(\bar{L}_{u_{r} \exp \left(t Y_{i}\right)}\right)_{*} Y_{i}^{*}\right|_{t=0}\right) .
$$

If $\operatorname{trace}_{\mathfrak{m}_{l}} U=0, \operatorname{trace}_{m_{l}} \nabla^{c} d f=\operatorname{trace}_{m_{l}} \nabla d f$ by Lemma 10.2. We have seen that the term involving $D^{c} / d t$ vanishes. This gives: 


$$
\left.\sum_{i=1}^{m_{l}} \frac{d^{2}}{d t^{2}} f \circ \pi\left(u_{r} \exp \left(t Y_{i}\right)\right)\right|_{t=0}=a^{2} \operatorname{trace}_{\mathfrak{m}_{l}} \nabla d f .
$$

Put everything together we see that

$$
\boldsymbol{E} f \circ \pi\left(u_{t}\right)=f \circ \pi\left(u_{0}\right)+\frac{1}{2} a^{2} \int_{0}^{t} \boldsymbol{E}(\text { trace })_{\mathfrak{m}_{l}} \nabla d f\left(\pi\left(u_{r}\right)\right) d r .
$$

For $x=\pi(y)$ we define $Q_{t} f(x)=P_{t}(f \circ \pi)(y)$, so $P_{t}(f \circ \pi)=\left(Q_{t} f\right) \circ \pi$. We apply Dynkin's criterion for functions of a Markov process to see that $\left(\bar{x}_{t}\right)$ is Markovian. The infinitesimal generator associated to $Q_{t}$ is $(1 / 2) a^{2}$ (trace) $\mathfrak{m}_{l} \nabla d f$. If $M$ is an irreducible Riemannian symmetric space, $\mathfrak{m}$ is irreducible, $d \pi(\mathfrak{m})=T_{o} M$ and the Markov process $\left(\bar{x}_{t}\right)$ is a scaled Brownian motion. This concludes the Proposition.

If $M$ is naturally reductive, the proof is even simpler. In this case, $\sigma_{i}(t)=$ $\pi\left(u \exp \left(t Y_{i}\right)\right)$ is a geodesic with initial velocity $Y_{i}$ and $\dot{\sigma}(t)=(d / d t) \pi_{*}\left(u \exp \left(t Y_{i}\right)\right)$. Consequently, the following also vanishes:

$$
\left.\frac{D}{d t}\left(\bar{L}_{u_{r} \exp \left(t Y_{i}\right)}\right)_{*}\left(Y_{i}^{*}\right)\right|_{t=0}=0 .
$$

\section{Examples.}

Corollary 9.2 applies to the example in Section 2.1, where $G=S U(2), H=U(1)$ and the $\operatorname{Ad}_{H}$-invariant space $\mathfrak{m}=\left\langle X_{2}, X_{3}\right\rangle$ is irreducible. For any $Y \in \mathfrak{m}, \operatorname{ad}^{2}\left(X_{1}\right)(Y)=$ $-4 Y$. Note that 4 is the second non-zero eigenvalue of $\Delta_{S^{1}}$, also the killing form of $S U(2)$ is $K(X, Y)=4 \operatorname{trace}(X Y)$ and $\mathcal{B}_{\mathrm{ad}_{\mathfrak{h}}, \mathfrak{m}}\left(X_{1}, X_{1}\right)=K\left(X_{1}, X_{1}\right)=-8$.

EXAmple 11.1. Let $\left(b_{t}\right)$ be a one dimensional Brownian motion, $g_{0} \in S U(2)$, $Y_{0} \in\left\langle X_{2}, X_{3}\right\rangle$ non-zero. Let $\left(g_{t}^{\epsilon}, h_{t}^{\epsilon}\right)$ be the solution to the following SDE on $S U(2) \times U(1)$,

$$
d g_{t}^{\epsilon}=g_{t}^{\epsilon} Y_{0} d t+\frac{1}{\sqrt{\epsilon}} g_{t}^{\epsilon} X_{1} d b_{t}
$$

with $g_{0}^{\epsilon}=g_{0}$. Let $\pi(z, w)=\left(\left(|w|^{2}-|z|^{2}\right) / 2, z \bar{w}\right)$ and $x_{t}^{\epsilon}=\pi\left(g_{t}^{\epsilon}\right)$. Let $\left(\tilde{x}_{t}^{\epsilon}\right)$ be the horizontal lift of $\left(x_{t}^{\epsilon}\right)$. Then $\left(\tilde{x}_{t / \epsilon}^{\epsilon}\right)$ converges weakly to the hypoelliptic diffusion with generator $\overline{\mathcal{L}}=\left(\left|Y_{0}\right|^{2} / 4\right) \Delta^{\text {hor }}$. Furthermore, $x_{t / \epsilon}^{\epsilon}$ converges in law to the Brownian motion on $S^{2}(1 / 2)$ scaled by $\left|Y_{0}\right|^{2} / 2$.

The first part of the theorem follows from Theorem 6.10 and Corollary 9.2. The scaling $1 / 2$ indicates the extra time needed for producing the extra direction $\left[X_{1}, Y_{0}\right]$. For the second part we use the fact that $G$ is compact, so $\langle[X, Y],[Z]\rangle=-\langle Y,[X, Z]\rangle$ for any $X, Y, Z \in \mathfrak{m}$, and Proposition 10.3 applies. Incidentally, a similar argument can be made for an analogous equation on $G=S U(n)$ and $H$ the torus group of $G$.

ExAmple 11.2. Let $G=S O(4), H=S O(3), E_{i, j}$ the elementary $4 \times 4$ matrices, and $A_{i, j}=\left(E_{i j}-E_{j i}\right) / \sqrt{2}$. Then $\mathfrak{h}=\left\{A_{1,2}, A_{1,3}, A_{2,3}\right\}$, its orthogonal complement $\mathfrak{m}$ is irreducible w.r.t. $\operatorname{Ad}_{H}$. For $k=1,2$, and 3 , let $Y_{k}=A_{k 4}$. Let us consider the equations, 


$$
d g_{t}^{\epsilon}=\frac{1}{\sqrt{\epsilon}} A_{1,2}\left(g_{t}^{\epsilon}\right) \circ d b_{t}^{1}+\frac{1}{\sqrt{\epsilon}} A_{1,3}\left(g_{t}^{\epsilon}\right) \circ d b_{t}^{2}+Y_{k}\left(g_{t}^{\epsilon}\right) d t
$$

Observe that $\left\{A_{1,2}, A_{1,3}\right\}$ is a set of generators, in fact $\left[A_{1,2}, A_{1,3}\right]=(-1 / \sqrt{2}) A_{2,3}$, and $\mathcal{L}_{0}=\left(A_{1,2}\right)^{2} / 2+\left(A_{1,3}\right)^{2} / 2$ satisfies strong Hörmander's conditions. It is easy to check that,

$$
\frac{1}{2} \operatorname{ad}^{2}\left(A_{1,2}\right)\left(Y_{k}\right)+\frac{1}{2} \operatorname{ad}^{2}\left(A_{1,3}\right)\left(Y_{k}\right)=-\frac{1}{4}\left(2 \delta_{1, k}+\delta_{2, k}+\delta_{3, k}\right) Y_{k} .
$$

For any $Y \in \mathfrak{m},\left\langle Y, \operatorname{Ad}(h) Y_{k}\right\rangle$ is an eigenfunction of $\mathcal{L}_{0}$ corresponding to the eigenvalue $-\lambda\left(Y_{k}\right)$, where

$$
\lambda\left(Y_{1}\right)=\frac{1}{2}, \lambda\left(Y_{2}\right)=\frac{1}{4}, \lambda\left(Y_{3}\right)=\frac{1}{4} .
$$

Define $\pi: g \in S O(4) \rightarrow S^{4}$ to be the map projecting $g$ to its last column. Set $x_{t}^{\epsilon}=\pi\left(g_{t}^{\epsilon}\right)$ whose horizontal lift through $u_{0}$ will be denoted by $\tilde{x}_{t}^{\epsilon}$. Then

$$
a_{i, j}\left(Y_{k}\right):=\frac{1}{\lambda\left(Y_{k}\right)} \int_{H}\left\langle Y_{i}, \operatorname{Ad}(h) Y_{k}\right\rangle \mathcal{L}_{0}^{-1}\left(\left\langle Y_{j}, \operatorname{Ad}(h)\left(Y_{k}\right)\right\rangle\right) d h .
$$

For $i \neq j, a_{i, j}\left(Y_{k}\right)=0$ and $a_{i, i}\left(Y_{k}\right):=1 / 3 \lambda\left(Y_{k}\right)$. In Theorem 6.10 take $Y_{0}=Y_{k}$ to see

$$
\overline{\mathcal{L}}=\frac{1}{\lambda\left(Y_{k}\right)} \int_{S O(3)} \nabla^{L} d f\left(\left(\operatorname{Ad}(h)\left(Y_{k}\right)\right)^{*},\left(\operatorname{Ad}(h)\left(Y_{k}\right)\right)^{*}\right) d h=\frac{1}{3 \lambda\left(Y_{k}\right)} \sum_{i=1}^{3} \nabla^{L} d f\left(Y_{i}, Y_{i}\right) .
$$

On the other hand, the effective diffusion for the equations

$$
d g_{t}^{\epsilon}=\frac{1}{\sqrt{\epsilon}} A_{1,2}\left(g_{t}^{\epsilon}\right) \circ d b_{t}^{1}+\frac{1}{\sqrt{\epsilon}} A_{1,3}\left(g_{t}^{\epsilon}\right) \circ d b_{t}^{2}+\frac{1}{\sqrt{\epsilon}} A_{2,3}\left(g_{t}^{\epsilon}\right) \circ d b_{t}^{3}+Y_{k}\left(g_{t}^{\epsilon}\right) d t
$$

is the same for all $Y_{k}$. It is easy to see that $\overline{\mathcal{L}}=(2 / 3) \sum_{i=1}^{3} \nabla^{L} d f\left(Y_{i}, Y_{i}\right)$.

Further symmetries can, of course, be explored.

EXAMPLE 11.3. Let $Y_{0}=\sum_{k=1}^{3} c_{k} Y_{k}$ be a mixed vector. Then,

$$
\overline{\alpha_{i} \beta_{j}}=\sum_{k, l=1}^{3} c_{k} c_{l} \overline{\alpha_{i}\left(Y_{k}\right) \beta_{j}\left(Y_{l}\right)}=\sum_{k, l=1}^{3} c_{k} c_{l} \frac{1}{\lambda_{l}} \overline{\alpha_{i}\left(Y_{k}\right) \alpha_{j}\left(Y_{l}\right)}
$$

If $c_{1}=0$ and $c_{2}=c_{3}, \overline{\alpha_{i} \beta_{j}}=4\left(c_{2}\right)^{2} \sum_{k, l=2}^{3} \overline{\alpha_{i}\left(Y_{k}\right) \alpha_{j}\left(Y_{l}\right)}$. By symmetry, $\overline{\alpha_{i}\left(Y_{k}\right) \alpha_{j}\left(Y_{l}\right)}$ vanishes for $i \neq j$.

ExAmple 11.4. Let $n \geq 2, G=S O(n+1), H=\left\{\left(\begin{array}{ll}R & 0 \\ 0 & 1\end{array}\right), R \in S O(n)\right\}$, and $S^{n}=S O(n+1) / S O(n)$. Then $H$ fixes the point $o=(0, \ldots, 0,1)^{T}$. The homogeneous space $S^{n}$ has the reductive decomposition: 


$$
\mathfrak{h}=\left\{\left(\begin{array}{ll}
S & 0 \\
0 & 0
\end{array}\right), S \in \mathfrak{s o}(n)\right\}, \mathfrak{m}=\left\{Y_{C}=\left(\begin{array}{cc}
0 & C \\
-C^{T} & 0
\end{array}\right), C \in \mathbb{R}^{n}\right\} .
$$

Let $\sigma(A)=S_{0} A S_{0}^{-1}$ and $S_{0}=\left(\begin{array}{cc}I & 0 \\ 0 & -1\end{array}\right)$. Then $\mathfrak{g}=\mathfrak{h} \oplus \mathfrak{m}$ is the symmetric space decomposition for $\sigma$. We identify $Y_{C}$ with the vector $C$, and compute:

$$
\operatorname{Ad}\left(\left(\begin{array}{ll}
R & 0 \\
0 & 1
\end{array}\right) Y_{C}\right)=\left(\begin{array}{cc}
0 & R C \\
-(R C)^{T} & 0
\end{array}\right) .
$$

This action is transitive on the unit tangent sphere and is irreducible. There is a matrix $R \in S O(n)$ that sends $C$ to $-C$. Then $\bar{Y}_{0}=0$ for every $Y_{0} \in \mathfrak{m}$ and the conditions of Proposition 8.2 are satisfied.

Let $A_{i, j}=(1 / \sqrt{2})\left(E_{i j}-E_{j i}\right)$, where $i<j$, and $E_{i j}$ is the elementary matrix with the nonzero entry at the $(i, j)$-th position. Let $Y_{0} \in \mathfrak{m}$ be a non-trivial vector. Consider the equation,

$$
d g_{t}^{\epsilon}=\frac{1}{\sqrt{\epsilon}} \sum_{1 \leq i<j \leq n} A_{i, j}^{*}\left(g_{t}^{\epsilon}\right) \circ d b_{t}^{i, j}+Y_{0}^{*}\left(g_{t}^{\epsilon}\right) d t .
$$

We define $\lambda=(n-1) / 4$. By a symmetry argument it is easy to see that,

$$
\sum_{1 \leq i<j \leq n} \operatorname{ad}^{2}\left(A_{i j}\right)=-\frac{1}{2}(n-1) I .
$$

For $1 \leq i, j, k \leq n,\left[A_{i, j}, A_{k, n+1}\right]=(-1 / \sqrt{2}) \delta_{k, i} A_{j, n+1}+(1 / \sqrt{2}) \delta_{j, k} A_{i, n+1}$, which follows from $E_{i j} E_{k l}=\delta_{j k} E_{i l}$. Hence for $i \neq j$,

$$
2 \operatorname{ad}^{2}\left(A_{i, j}\right)\left(A_{k, n+1}\right)=-\left(\delta_{i, k}+\delta_{j, k}\right) A_{k, n+1} .
$$

Let $x_{t / \epsilon}^{\epsilon}=g_{t / \epsilon}^{\epsilon} O$ and $\left(\tilde{x}_{t / \epsilon}^{\epsilon}\right)$ the horizontal lift of $\left(x_{t / \epsilon}^{\epsilon}\right)$ through $g_{0}$. By Theorem 6.10, converges to a Markov process whose limiting Markov generator is, by symmetry,

$$
\overline{\mathcal{L}}=\frac{\left|Y_{0}\right|^{2}}{\lambda \operatorname{dim}(\mathfrak{m})} \Delta^{h o r}=\frac{4\left|Y_{0}\right|^{2}}{n(n-1)} \Delta^{h o r} .
$$

The upper bound for the rate of convergence from Theorem 6.5 holds. By Theorem 6.10 and Proposition 10.3 the stochastic processes $\left(x_{t / \epsilon}^{\epsilon}\right)$ converge to the Brownian motion on $S^{n}$ with scale $8\left|Y_{0}\right|^{2} / n(n-1)$. By $(11.2)$, for any $1 \leq i, j, k \leq n, A_{k, n+1}$ are eigenvectors for $\operatorname{ad}^{2}\left(A_{i, j}\right)$. Furthermore, for $1 \leq i<j \leq n$,

$$
\left[A_{i, n+1}, A_{j, n+1}\right]=-\frac{1}{\sqrt{2}} A_{i, j},
$$

and $\Delta^{\text {hor }}$ satisfies the one step Hörmander condition.

EXAMPLE 11.5. We keep the notation in the example above. Let $Y_{0}$ be a unit vector from $\mathfrak{m}$ and let $\left(g_{t}^{\epsilon}\right)$ be the solutions of the following equation: 


$$
d g_{t}^{\epsilon}=\frac{1}{\sqrt{\epsilon}} \sum_{1 \leq i<j \leq n} A_{i, j}^{*}\left(g_{t}^{\epsilon}\right) \circ d b_{t}^{i, j}+\frac{n(n-1)}{8} Y_{0}^{*}\left(g_{t}^{\epsilon}\right) d t .
$$

Then as $\epsilon \rightarrow 0, \pi\left(g_{t / \epsilon}^{\epsilon}\right)$ converges to a Brownian motion on $S^{n}$.

We finally provide an example in which the group $G$ is not compact.

ExAMPLE 11.6. Let $n \geq 3$. Let $F(x, y)=-x_{0} y_{0}+\sum_{k=1}^{n} x_{k} y_{k}$ be a bilinear form on $\mathbb{R}^{n+1}$. Let $O(1, n)$ be the set of $(n+1) \times(n+1)$ matrices preserving the indefinite form

$$
O(1, n)=\left\{A \in G L(n+1): A^{T} S A=S, S=\left(\begin{array}{cc}
-1 & 0 \\
0 & I_{n}
\end{array}\right)\right\}
$$

Let $G$ denote the identity component of $O(1, n)$, consisting of $A \in O(1, n)$ with $\operatorname{det}(A)=$ 1 and $a_{00} \geq 1$. This is an $n(n+1) / 2$ dimensional manifold, $X \in \mathfrak{g}$ if and only if $X^{T} S+S X=0$. So

$$
\mathfrak{g}=\left\{\left(\begin{array}{cc}
0 & \xi^{T} \\
\xi & S
\end{array}\right), \xi \in \mathbb{R}^{n}, S \in \mathfrak{s o}(n)\right\} .
$$

The map $\sigma(A)=S A S^{-1}$ defines an evolution on $G$ and

$$
M=\left\{x=\left(x^{0}, x^{1}, \ldots, x^{n}\right): F(x, x)=-1, x^{0} \geq 1\right\}
$$

is a symmetric space. Its isometry group at $e_{0}=(1,0, \ldots, 0)^{T}$ is $H=\left\{\left(\begin{array}{ll}1 & 0 \\ 0 & B\end{array}\right)\right\}$ where $B \in S O(n)$. The -1 eigenspace of the Lie algebra involution is:

$$
\mathfrak{m}=\left\{\left(\begin{array}{cc}
0 & \xi^{T} \\
\xi & 0
\end{array}\right)\right\} .
$$

Let us take $Y_{0} \in \mathfrak{m}$ and consider the equation,

$$
d g_{t}^{\epsilon}=\frac{1}{\sqrt{\epsilon}} \sum_{1 \leq i<j \leq n} A_{i, j}^{*}\left(g_{t}^{\epsilon}\right) \circ d b_{t}^{i, j}+Y_{0}^{*}\left(g_{t}^{\epsilon}\right) d t .
$$

It is clear that Theorems 6.10 and 7.3 apply. The isotropy representation of $H$ is irreducible. The action of $\operatorname{Ad}_{H}$ on $\mathfrak{m}$ is essentially the action of $S O(n)$ on $\mathbb{R}^{n}$ :

$$
\operatorname{Ad}\left(\left(\begin{array}{ll}
1 & 0 \\
0 & B
\end{array}\right)\right)\left(\begin{array}{cc}
0 & \xi^{T} \\
\xi & 0
\end{array}\right)=\left(\begin{array}{cc}
0 & (B \xi)^{T} \\
B \xi & 0
\end{array}\right) .
$$

The conclusion of Theorems hold. The symmetric space $M$ is naturally reductive and so Proposition 10.3 applies. Note that

$$
\left[\left(\begin{array}{cc}
0 & \xi^{T} \\
\xi & 0
\end{array}\right),\left(\begin{array}{cc}
0 & \eta^{T} \\
\eta & 0
\end{array}\right)\right]=\left(\begin{array}{cc}
0 & 0 \\
0 & \xi \eta^{T}-\eta \xi^{T}
\end{array}\right) .
$$

Since $e_{i} e_{j}^{T}=E_{i j},[\mathfrak{m}, \mathfrak{m}]$ generates a basis of $\mathfrak{h}$. The effective process arising from the 
family of stochastic differential equations (1.1) is a scaled horizontal Brownian motion. Observe that the latter satisfies the one step Hörmander condition. The limit of $\pi\left(g_{t / \epsilon}^{\epsilon}\right)$ is a scaled hyperbolic Brownian motion. The rate of convergence stated in Theorem 6.5 is valid here, the scale is $8 / n(n-1)$.

\section{Further discussions and open questions.}

The study of scaling limits should generalise to principal bundles and to foliated manifolds. Let $\pi: P \rightarrow M$ be a principal bundle with group action $H$ whose Lie algebra $\mathfrak{h}$ is given a suitable inner product. For $A \in \mathfrak{h}$ denote by $A^{*}$ the corresponding fundamental vertical vector field: $A^{*}(u)=\left.(d / d t)\right|_{t=0} u \exp (t A)$. Let us fix an orthonormal basis $\left\{A_{1}, \ldots, A_{p}\right\}$ of $\mathfrak{h}$. Let $\left\{\sigma_{j}, j=p+1, \ldots, n\right\}$ be smooth horizontal sections of $T P, f_{0}$ a vertical vector field, $\left\{w_{t}^{1}, \ldots, w_{t}^{N_{1}}, b_{t}^{1}, \ldots, b_{t}^{N_{2}}\right\}$ independent one dimensional Brownian motions. Let $\left\{a_{j}^{k}, c_{i}^{l}\right\}$ be a family of smooth functions on $P$. For example, a computation analogous to that in Lemma 4.1, should lead to a system of SDEs of Markovian type, whose solutions are slow and fast motions, and the following proposition.

Proposition 12.1. Let $u_{t} \equiv \phi_{t}\left(u_{0}\right)$ be a solution to

$$
d u_{t}=\left(\sigma_{0}+f_{0}\right)\left(u_{t}\right) d t+\sum_{k=1}^{N_{1}} \sum_{j=p+1}^{n}\left(c_{k}^{j} \sigma_{j}\right)\left(u_{t}\right) \circ d w_{t}^{k}+\sum_{l=1}^{N_{2}} \sum_{j=1}^{p}\left(a_{l}^{j} A_{j}^{*}\right)\left(u_{t}\right) \circ d b_{t}^{l} .
$$

Set $x_{t}=\pi\left(u_{t}\right)$ and denote by $\left(\tilde{x}_{t}\right)$ its horizontal lift. Then $u_{t}=\tilde{x}_{t} a_{t}$ where

$$
\begin{aligned}
& d \tilde{x}_{t}=\left(R_{a_{t}^{-1}}\right)_{*} \sigma_{0}\left(\tilde{x}_{t}\right) d t+\sum_{k=1}^{N_{2}} \sum_{j=p+1}^{n} c_{k}^{j}\left(\tilde{x}_{t} a_{t}\right)\left(R_{a_{t}^{-1}}\right)_{*}\left(\sigma_{j}\right)\left(\tilde{x}_{t}\right) \circ d w_{t}^{k} \\
& d a_{t}=T R_{a_{t}}\left(\varpi_{\tilde{x}_{t}}\left(f_{0}\right)\right) d t+\sum_{l=1}^{N_{1}} \sum_{j=1}^{p} a_{l}^{j}\left(\tilde{x}_{t} a_{t}\right) A_{j}^{*}\left(a_{t}\right) \circ d b_{t}^{l} .
\end{aligned}
$$

If each $c_{k}^{j}$ vanishes identically the problem is easier in which case we are led naturally to random ODEs of the following type

$$
\begin{aligned}
\dot{y}_{t}^{\epsilon} & =\sum_{k=1}^{m} Y_{k}\left(y_{t}^{\epsilon}\right) \alpha_{k}\left(z_{t}^{\epsilon}(\omega), y_{t}^{\epsilon}\right) \\
y_{0}^{\epsilon} & =y_{0} .
\end{aligned}
$$

We may assume that the stochastic processes $\left(z_{t}^{\epsilon}\right)$ are hypoelliptic diffusions or Lévy processes satisfying a Birkhoff's ergodic theorem, and $\alpha_{k}: G \times M \rightarrow \mathbb{R}$ are smooth functions. The vector fields $\left\{Y_{k}\right\}$ are, for example, given by $Y_{k}(\cdot)=Y(\cdot)\left(e_{k}\right)$ where for each $y \in P, Y(y): \mathbb{R}^{m} \rightarrow T_{y} P$ is isometric and $\left\{e_{k}\right\}$ is an orthonormal basis of $\mathbb{R}^{m}$. Letting $\epsilon$ approaches zero, we expect an averaging principle and an effective ODE in the limit, c.f. [45]. For the next step, the challenging problem is to find geometrically meaningful conditions on the coefficients so that the effective ODE is trivial, in which case one may proceed to obtain an effective diffusion on a larger time scale. We observe 
that the limit theorems in $[\mathbf{4 7}]$ do not cover this.

ACKnOwledgments. I would like to acknowledge helpful conversations with K. Ardakov, R. L. Bryant, K. D. Elworthy, M. Hairer, J. Rawnsley and D. Rumynin. I also like to thank the Institute for Advanced Studies for its hospitality during my visit in 2014 .

\title{
Appendix: Real Peter-Weyl by Dmitriy Rumynin
}

\begin{abstract}
We explain how unitary representations of compact groups split into three types. We summarise their properties. As an application we state and prove Peter-Weyl Theorem for real representations of compact groups.
\end{abstract}

Let $G$ be a compact topological group. We refer the reader to textbooks $[\mathbf{7 0}],[\mathbf{7 1}]$ for standard properties of $G$ and its representations. The group $G$ has a unique biinvariant Haar measure $\mu$ such that $\mu(G)=1$. We utilise the corresponding integral $\int_{G} \Phi(x) \mu(d x)$.

We study unitary representations of $G$. It is a representation of $G$ on a real (or complex) Hilbert space $V$ (or $\mathcal{V}$ - we use calligraphic letters for complex Hilbert spaces) such that the action map $G \times V \rightarrow V$ is continuous and each $g \in G$ acts by an orthogonal (unitary) operator. A key example is the real regular representation $L^{2}(G)$, that is, the space of all $\mathbb{R}$-valued $L^{2}$-functions with the action $[x \cdot \Phi](y)=\Phi(y x)$. By $\mathcal{L}^{2}(G)$ we denote the complex regular representation, the space of all $\mathbb{C}$-valued $L^{2}$-functions with the same action.

Each unitary representation is a direct sum of irreducible unitary representations. Each irreducible unitary representation is finite dimensional. Let $\operatorname{Irr}_{\mathbb{R}} G$ be the set of isomorphism classes of irreducible unitary representations (correspondingly $\operatorname{Irr}_{\mathbb{C}} G$ for complex ones). The character of $V \in \operatorname{Irr}_{\mathbb{R}} G$ is the function $\chi_{V} \in L^{2}(G)$ given by $\chi_{V}(x)=\operatorname{Tr}_{V}(x)$. The Frobenius-Schur indicator of $\mathcal{V} \in \operatorname{Irr}_{\mathbb{C}} G\left(\right.$ or $\left.V \in \operatorname{Irr}_{\mathbb{R}} G\right)$ is

$$
\operatorname{FS}(\mathcal{V})=\int_{G} \chi_{\mathcal{V}}\left(x^{2}\right) \mu(d x)\left(\text { or } \operatorname{FS}(V)=\int_{G} \chi_{V}\left(x^{2}\right) \mu(d x)\right) .
$$

A complex unitary representation $(\mathcal{V},\langle\mid\rangle)$ can be considered as a real vector space $\mathcal{V}_{\mathbb{R}}$. It is a real unitary representation with a form $\operatorname{Re}\langle\mid\rangle$. In the opposite direction, $V \otimes_{\mathbb{R}} \mathbb{C}$ is a complexification of $(V,\langle\mid\rangle)$ with a natural unitary form $\langle x \otimes \alpha \mid y \otimes \beta\rangle=\bar{\alpha} \beta\langle x \mid y\rangle$. Notice that $\left(V \otimes_{\mathbb{R}} \mathbb{C}\right)_{\mathbb{R}}=V \otimes 1 \oplus V \otimes \boldsymbol{i} \cong 2 V$ as real representations of $G$. This defines a correspondence

$$
\operatorname{Irr}_{\mathbb{R}} G \leftrightarrow \operatorname{Irr}_{\mathbb{C}} G .
$$

The representations $V$ and $\mathcal{V}$ are in correspondence if $\mathcal{V}$ is a direct summand of $V \otimes_{\mathbb{R}} \mathbb{C}$. By Frobenius reciprocity, this is equivalent to $V$ being a direct summand of $\mathcal{V}_{\mathbb{R}}$. The properties of this correspondence depend on the endomorphism algebra $\mathbb{F}=\operatorname{End}_{G}(V)$. Recall that $\mathbb{F}$ consists of all $\mathbb{R}$-linear operators on $V$ that commute with all elements of $G$. 
The algebra $\mathbb{F}$ is a division algebra (Schur lemma), hence, it must be either real numbers $\mathbb{R}$, or complex numbers $\mathbb{C}$, or quaternions $\mathbb{H}$ (Frobenius Theorem). Depending on what $\mathbb{F}$ is, we call each of the representations in correspondence $V \leftrightarrow \mathcal{V}$ a representation of real, complex or quaternionic type correspondingly.

Useful properties of representations of each type are summarised in Table 1 . The last two rows show that the type can be determined by computing the Frobenius-Schur indicator: it is particularly useful for a finite group $G$.

Table 1. Properties of $V \leftrightarrow \mathcal{V}$ depending on $\mathbb{F}$.

\begin{tabular}{|c||c|c|c|}
\hline $\mathbb{F}=\operatorname{End}_{G}(V)$ & $\mathbb{R}$ & $\mathbb{C}$ & $\mathbb{H}$ \\
\hline$V_{\mathbb{C}} \cong \mathcal{V}$ & Yes & No & No \\
$V_{\mathbb{C}}$ & $\mathcal{V}$ & $\mathcal{V} \oplus \mathcal{V}^{*}$ & $2 \mathcal{V}$ \\
$\operatorname{Irr}_{\mathbb{R}} G$ to $\operatorname{Irr}_{\mathbb{C}} G$ correspondence is & $1: 1$ & $1: 2$ & $1: 1$ \\
$\mathcal{V}_{\mathbb{R}}$ & $2 V$ & $V$ & $V$ \\
$\chi_{\mathcal{V}}$ takes values in & $\mathbb{R}$ & $\mathbb{C}$ & $\mathbb{R}$ \\
relation between characters & $\chi_{V}=\chi_{\mathcal{V}}$ & $\chi_{V}=2 \cdot \operatorname{Re}\left(\chi_{\mathcal{V}}\right)$ & $\chi_{V}=2 \cdot \chi_{\mathcal{V}}$ \\
$\mathcal{V} \cong \mathcal{V}^{*}$ & Yes & No & Yes \\
$G$-invariant form on $\mathcal{V}$ & symmetric & only zero & skew-symmetric \\
$\operatorname{FS}(\mathcal{V})$ is equal to & 1 & 0 & -1 \\
$\operatorname{FS}(V)$ is equal to & 1 & 0 & -2 \\
\hline
\end{tabular}

The third and fourth rows from the bottom are useful for a simple compact Lie group $G$. An irreducible representation $\mathcal{V}$ of $G$ is parametrised by its highest weight $\varpi$ : we write $\mathcal{V}=\mathcal{L}(\varpi)$, if $\mathcal{V}$ is an irreducible representation with a highest weight vector of weight $\varpi$. It is known that $\mathcal{L}(\varpi)^{*}=\mathcal{L}(-w \cdot \varpi)$ where $w$ is the longest element of the Weyl group. The fourth row immediately tells you whether $V \leftrightarrow \mathcal{V}$ is of complex type or not: it is easy to verify whether $\varpi=-w \cdot \varpi$ or not in each particular case. For instance, the vector representation of $S U_{n}(\mathbb{C})$ is $\mathcal{L}\left(\varpi_{1}\right)$ where $\varpi_{1}$ is the first fundamental weight. Then $\mathcal{L}\left(\varpi_{1}\right)^{*}=\mathcal{L}\left(-w \cdot \varpi_{1}\right)=\mathcal{L}\left(\varpi_{n-1}\right)$ so that $\mathcal{L}\left(\varpi_{1}\right)$ is of complex type if $n>2$.

Look at $S U_{2}(\mathbb{C})$. Its irreducible representations are $\mathcal{L}\left(m \varpi_{1}\right)$ for natural $m$ and $\mathcal{L}\left(m \varpi_{1}\right)^{*}=\mathcal{L}\left(-w \cdot m \varpi_{1}\right)=\mathcal{L}(m \varpi)$. No representation of $S U_{2}(\mathbb{C})$ is of complex type. The vector representation $\mathcal{L}\left(\varpi_{1}\right)$ admits a symplectic form preserved by $S U_{2}(\mathbb{C})$. The representation $\mathcal{L}\left(m \varpi_{1}\right)$ is isomorphic to $S^{m}\left(\mathcal{L}\left(\varpi_{1}\right)\right)$, its $m$-th symmetric power, that gets an induced $S U_{2}(\mathbb{C})$-invariant form, symmetric if $m$ is even, skew-symmetric if $m$ is odd. Thus, $\mathcal{L}\left(m \varpi_{1}\right)$ is of real type if $m$ is even and is of quaternionic type if $m$ is odd.

The first row helps to determine the type of the adjoint representation $\mathfrak{g}$ of a compact simple Lie group $G$. Its complexification $\mathfrak{g} \otimes_{\mathbb{R}} \mathbb{C}$ is a simple complex Lie algebra. So it is of real type. Alternatively one can use its Killing form or the fact that $\mathfrak{g} \otimes_{\mathbb{R}} \mathbb{C}=\mathcal{L}\left(\alpha_{0}\right)$ where $\alpha_{0}$ is the highest root.

The field $\mathbb{F}$ is a "natural" field for $V$. We will write elements of $\mathbb{F}$ acting on vectors from the right (it is essential since $\mathbb{H}$ is non-commutative). 
Lemma 1 (Real Artin-Wedderburn Formula). $\quad L^{2}(G) \cong \bigoplus_{V \in \operatorname{Irr}_{\mathbb{R}} G} \operatorname{dim}_{\mathbb{F}}(V) V$.

Proof. $\quad$ Notice that $L^{2}(G) \otimes_{\mathbb{R}} \mathbb{C} \cong \mathcal{L}^{2}(G) \cong \bigoplus_{\mathcal{V} \in \operatorname{Irr}_{\mathbb{C}} G} \operatorname{dim}_{\mathbb{C}}(\mathcal{V}) \mathcal{V}$. Thus, we need to prove that $\operatorname{dim}_{\mathbb{F}}(V) V \otimes_{\mathbb{R}} \mathbb{C}$ is isomorphic to $\bigoplus_{V \leftrightarrow \mathcal{V}} \operatorname{dim}_{\mathbb{C}}(\mathcal{V}) \mathcal{V}$. This is easily checked case by case for each column in Table 1 .

We fix standard imaginary units $\boldsymbol{i}, \boldsymbol{j}, \boldsymbol{k} \in \mathbb{H}$ and $\boldsymbol{i} \in \mathbb{C}$. The complex conjugation on $\mathbb{F}$ is given by the formula $\overline{\alpha+\beta \boldsymbol{i}+\gamma \boldsymbol{j}+\delta \boldsymbol{k}}=\alpha-\beta \boldsymbol{i}-\gamma \boldsymbol{j}-\delta \boldsymbol{k}$. Note that it is identity if $\mathbb{F}=\mathbb{R}$. Given an $\mathbb{F}$-valued function $\Phi$, we want to fix its one, two or four (depending on $\mathbb{F}$ ) components, the $\mathbb{R}$-valued functions $\Phi^{\bullet}$ such that

$$
\Phi=\Phi^{1} 1+\Phi^{i} \boldsymbol{i}+\Phi^{j} \boldsymbol{j}+\Phi^{\boldsymbol{k}} \boldsymbol{k} .
$$

Now we are ready to exhibit an explicit orthonormal basis of $L^{2}(G)$, e.g. state Peter-Weyl Theorem. Let $(V,\langle\mid\rangle) \in \operatorname{Irr}_{\mathbb{R}} G, d=\operatorname{dim}_{\mathbb{F}}(V)$. Consider $V$ as a right vector space over $\mathbb{F}$. Choose a vector space isomorphism with the space of columns

$$
V \rightarrow \mathbb{F}^{d}, a \mapsto \underline{a} .
$$

This gives a $G$-invariant positive definite unitary $\mathbb{F}$-form on $V$ :

$$
[a \mid b]=\int_{G}(\underline{x \cdot a})^{*} \underline{x \cdot b} \mu(d x)
$$

where $(\underline{x \cdot a})^{*}$ is the conjugate-transpose row of the column $\underline{x \cdot a}$. Observe that $[a \boldsymbol{q} \mid b \boldsymbol{r}]=$ $\overline{\boldsymbol{q}}[a \mid b] \boldsymbol{r}$ for all $\boldsymbol{q}, \boldsymbol{r} \in \mathbb{F}$.

Lemma 2 (Real Schur Lemma). Let $\{\mid\}$ be a $G$-invariant unitary $\mathbb{F}$-form on $V$. Then there exists $\boldsymbol{r} \in Z(\mathbb{F})$ (the centre of $\mathbb{F}$ ) such that

$$
\{a \mid b\}=\boldsymbol{r}[a \mid b] \text { for all } a, b \in V .
$$

Let $\{\mid\}^{\sharp}$ be a $G$-invariant bilinear $\mathbb{R}$-form on $V$. Then there exists $\boldsymbol{q} \in \mathbb{F}$ such that

$$
\{a \mid b\}^{\sharp}=\langle a \mid b \boldsymbol{q}\rangle \text { for all } a, b \in V \text {. }
$$

Finally let $\{\mid\}^{b}$ be a $G$-invariant symmetric bilinear $\mathbb{R}$-form on $V$. Then there exists $\boldsymbol{r} \in \mathbb{R}$ such that

$$
\{a \mid b\}^{b}=\boldsymbol{r}\langle a \mid b\rangle \text { for all } a, b \in V \text {. }
$$

Proof. The linear operator $\theta: V \rightarrow V$ defined by the equation

$$
\{a \mid b\}=[a \mid \theta(b)] \text { for all } a, b \in V
$$

is a $G$-module endomorphism since both form are $G$-invariant. Thus, $\theta(a)=a \boldsymbol{r}$ for some $\boldsymbol{r} \in \mathbb{F}$ and $\{a \mid b\}=[a \mid b] \boldsymbol{r}$. The unitarity of $\{\mid\}$ ensures that $\boldsymbol{r}$ is in the centre.

A proof for $\{\mid\}^{b}$ is similar. 
Using the second statement we can find $\boldsymbol{q} \in \mathbb{F}$ such that $\{a \mid b\}^{b}=\langle a \mid b \boldsymbol{q}\rangle$ for all $a, b$. Since $\{a \mid b\}^{b}$ is symmetric, $\boldsymbol{q}$ is self-adjoint:

$$
\langle a \mid b \boldsymbol{q}\rangle=\{a \mid b\}^{b}=\{b \mid a\}^{b}=\langle b \mid a \boldsymbol{q}\rangle=\langle a \boldsymbol{q} \mid b\rangle .
$$

It remains to notice that the adjoint of $\boldsymbol{q}$ is $\overline{\boldsymbol{q}}$. This proves that $\boldsymbol{q}=\overline{\boldsymbol{q}}$ and $\boldsymbol{q} \in \mathbb{R}$.

Notice that the component []$^{1}$ is a $G$-invariant positive definite symmetric bilinear $\mathbb{R}$-form. Lemma 2 implies that $[\mid]^{1}=\langle\mid\rangle \boldsymbol{r}$ for some positive $\boldsymbol{r} \in \mathbb{R}_{>0}$. Replacing [l] with $[\mid] \boldsymbol{r}^{-1}$, we get useful properties connecting the two forms.

LEMMA 3. If the relevant elements of $\mathbb{F}$ exist, then the following identities hold for all $a, b \in V:$

$$
\begin{gathered}
\langle a \mid b\rangle=[a \mid b]^{1}=\langle a \boldsymbol{i} \mid b \boldsymbol{i}\rangle=\langle a \boldsymbol{j} \mid b \boldsymbol{j}\rangle=\langle a \boldsymbol{k} \mid b \boldsymbol{k}\rangle, \\
\langle a \boldsymbol{i} \mid b\rangle=[a \mid b]^{\boldsymbol{i}}=-\langle a \mid b \boldsymbol{i}\rangle=-\langle a \boldsymbol{j} \mid b \boldsymbol{k}\rangle=\langle a \boldsymbol{k} \mid b \boldsymbol{j}\rangle, \\
-\langle a \mid b \boldsymbol{j}\rangle=[a \mid b]^{\boldsymbol{j}}=\langle a \boldsymbol{i} \mid b \boldsymbol{k}\rangle=-\langle a \boldsymbol{k} \mid b \boldsymbol{i}\rangle=\langle a \boldsymbol{j} \mid b\rangle, \\
-\langle a \mid b \boldsymbol{k}\rangle=[a \mid b]^{\boldsymbol{k}}=-\langle a \boldsymbol{i} \mid b \boldsymbol{j}\rangle=\langle a \boldsymbol{j} \mid b \boldsymbol{i}\rangle=\langle a \boldsymbol{k} \mid b\rangle .
\end{gathered}
$$

Proof. The first identity in the first row is a result of our assumptions. Now $[a \boldsymbol{i} \mid b]=[a \boldsymbol{i} \mid b]^{1} 1+[a \boldsymbol{i} \mid b]^{\boldsymbol{i}} \boldsymbol{i}+[a \boldsymbol{i} \mid b]^{\boldsymbol{j}} \boldsymbol{j}+[a \boldsymbol{i} \mid b]^{\boldsymbol{k}} \boldsymbol{k}$, while at the same time $[a \boldsymbol{i} \mid b]=-\boldsymbol{i}[a \mid b]=$ $[a \mid b]^{\boldsymbol{i}} 1-[a \mid b]^{1} \boldsymbol{i}+[a \mid b]^{\boldsymbol{k}} \boldsymbol{j}-[a \mid b]^{\boldsymbol{j}} \boldsymbol{k}$. This proves the first identity in the second row. The remaining proofs are similar using properties such as $[a \boldsymbol{j} \mid b \boldsymbol{k}]=-\boldsymbol{j}[a \mid b] \boldsymbol{k}$.

Let $X_{1}, X_{2}, \ldots, X_{m}$ be an orthonormal (with respect to [l]) $\mathbb{F}$-basis of $V$. The scaled coordinate functions in this basis are $\mathbb{F}$-valued functions on $G$

$$
{ }^{V} \Phi_{n, l}={ }^{V} \Phi_{n, l}^{1} 1+{ }^{V} \Phi_{n, l}^{i} \boldsymbol{i}+{ }^{V} \Phi_{n, l}^{\boldsymbol{j}} \boldsymbol{j}+{ }^{V} \Phi_{n, l}^{\boldsymbol{k}} \boldsymbol{k}, \quad{ }^{V} \Phi_{n, l}(g)=\sqrt{\operatorname{dim}_{\mathbb{R}}(V)}\left[X_{n} \mid g \cdot X_{l}\right] .
$$

Theorem 1 (Peter-Weyl Theorem). The components of the scaled coordinate functions $\left\{{ }^{V} \Phi_{n, l}^{\bullet} \mid V \in \operatorname{Irr}_{\mathbb{R}} G\right\}$ form an orthonormal basis of $L^{2}(G)$.

Proof. We deduce this theorem from the usual (complex) Peter-Weyl Theorem. This states that by equipping each $\mathcal{V} \in \operatorname{Irr}_{\mathbb{C}} G$ with an orthonormal basis $Y_{1}, Y_{2}, \ldots$ we obtain an orthonormal basis of $\mathcal{L}^{2}(G)$ consisting of scaled coordinate functions

$$
\left\{{ }^{\mathcal{V}} \Psi_{n, l} \mid \mathcal{V} \in \operatorname{Irr}_{\mathbb{C}} G\right\} \text { where }{ }^{\mathcal{V}} \Psi_{n, l}(g)=\sqrt{\operatorname{dim}_{\mathbb{C}}(\mathcal{V})}\left\langle Y_{n} \mid g \cdot Y_{k}\right\rangle
$$

If we fix $\mathcal{V}$, the functions ${ }^{\mathcal{V}} \Psi_{n, l}$ form an orthonormal basis of $\operatorname{dim}_{\mathbb{C}}(\mathcal{V}) \mathcal{V}$, a direct summand of $\mathcal{L}^{2}(G)$. It follows that ${ }^{V} \Phi_{n, l}^{\bullet}$ and ${ }^{W} \Phi_{n, l}^{\bullet}$ for non-isomorphic $V$ and $W$ are orthogonal to each other. It remains to observe that ${ }^{V} \Phi_{n, l}^{\bullet}$ form an orthonormal basis of $\operatorname{dim}_{\mathbb{F}}(V) V$. Let $d=\operatorname{dim}_{\mathbb{R}}(V)$.

If $\mathbb{F}=\mathbb{R}$, we can choose $Y_{n}=X_{n} \otimes 1$ as an orthonormal basis of $\mathcal{V}=V \otimes_{\mathbb{R}} \mathbb{C}$. Since $d=\operatorname{dim}_{\mathbb{C}}(\mathcal{V}),{ }^{V} \Phi_{n, l}^{1}=\mathcal{V} \Psi_{n, l}$ form an orthonormal basis.

If $\mathbb{F}=\mathbb{C}$, we have two complex representations $\mathcal{V}_{+}$and $\mathcal{V}_{-} \cong \mathcal{V}_{+}^{*}$ that we can explicitly pinpoint, writing the two idempotents of $\operatorname{End}_{G}\left(V \otimes_{\mathbb{R}} \mathbb{C}\right)=\mathbb{F} \otimes_{\mathbb{R}} \mathbb{C} \cong \mathbb{C}^{2}$ : 
$V \otimes_{\mathbb{R}} \mathbb{C}=\mathcal{V}_{-} \oplus \mathcal{V}_{+}$where $\mathcal{V}_{ \pm}=\left(V \otimes_{\mathbb{R}} \mathbb{C}\right) \boldsymbol{e}_{ \pm}, \quad \boldsymbol{e}_{ \pm}=\frac{1}{2}(1 \otimes 1 \pm \boldsymbol{i} \otimes \boldsymbol{i})$

Now we choose $Y_{n}^{ \pm}=\sqrt{2} X_{n} \boldsymbol{e}_{ \pm}$as an orthonormal basis of $\mathcal{V}_{ \pm}$. It is clear that $\left\langle Y_{n}^{+} \mid Y_{l}^{-}\right\rangle=$ 0 , while inside $\mathcal{V}_{ \pm}$

$$
\begin{aligned}
\left\langle Y_{n}^{ \pm} \mid Y_{k}^{ \pm}\right\rangle & =\frac{1}{2}\left\langle X_{n} \otimes 1 \pm X_{n} \boldsymbol{i} \otimes \boldsymbol{i} \mid X_{l} \otimes 1 \pm X_{l} \boldsymbol{i} \otimes \boldsymbol{i}\right\rangle \\
& =\frac{1}{2}\left\langle X_{n} \mid X_{l}\right\rangle \mp \frac{\boldsymbol{i}}{2}\left\langle X_{n} \boldsymbol{i} \mid X_{l}\right\rangle \pm \frac{\boldsymbol{i}}{2}\left\langle X_{n} \mid X_{l} \boldsymbol{i}\right\rangle+\frac{1}{2}\left\langle X_{n} \boldsymbol{i} \mid X_{l} \boldsymbol{i}\right\rangle \\
& =\frac{1}{2}\left[X_{n} \mid X_{l}\right]^{1} \mp \frac{\boldsymbol{i}}{2}\left[X_{n} \mid X_{l}\right]^{\boldsymbol{i}} \mp \frac{\boldsymbol{i}}{2}\left[X_{n} \mid X_{l}\right]^{\boldsymbol{i}}+\frac{1}{2}\left[X_{n} \mid X_{l}\right]^{1} \\
& =\left[X_{n} \mid X_{l}\right]^{1} \mp \boldsymbol{i}\left[X_{n} \mid X_{l}\right]^{\boldsymbol{i}}=\left[X_{n} \mid X_{l}\right]\left(\text { or } \overline{\left[X_{n} \mid X_{l}\right]}\right)=\delta_{n, l} .
\end{aligned}
$$

In this case $d=2 \operatorname{dim}_{\mathbb{C}} \mathcal{V}_{ \pm}$. We express the functions now:

$$
\begin{aligned}
\mathcal{V}_{ \pm} \Psi_{n, l}(g) & =\sqrt{\operatorname{dim}_{\mathbb{C}}\left(\mathcal{V}_{ \pm}\right)}\left\langle Y_{n}^{ \pm} \mid g \cdot Y_{k}^{ \pm}\right\rangle \\
& =\frac{\sqrt{d}}{2 \sqrt{2}}\left\langle X_{n} \otimes 1 \pm X_{n} \boldsymbol{i} \otimes \boldsymbol{i} \mid g \cdot X_{l} \otimes 1 \pm g \cdot X_{l} \boldsymbol{i} \otimes \boldsymbol{i}\right\rangle \\
& =\frac{\sqrt{d}}{2 \sqrt{2}}\left\langle X_{n} \mid g \cdot X_{l}\right\rangle \mp \frac{\boldsymbol{i} \sqrt{d}}{2 \sqrt{2}}\left\langle X_{n} \boldsymbol{i} \mid g \cdot X_{l}\right\rangle \pm \frac{\boldsymbol{i} \sqrt{d}}{2 \sqrt{2}}\left\langle X_{n} \mid g \cdot X_{l} \boldsymbol{i}\right\rangle+\frac{\sqrt{d}}{2 \sqrt{2}}\left\langle X_{n} \boldsymbol{i} \mid g \cdot X_{l} \boldsymbol{i}\right\rangle \\
& =\frac{\sqrt{d}}{2 \sqrt{2}}\left[X_{n} \mid g \cdot X_{l}\right]^{1} \mp \frac{\boldsymbol{i} \sqrt{d}}{2 \sqrt{2}}\left[X_{n} \mid g \cdot X_{l}\right]^{\boldsymbol{i}} \mp \frac{\boldsymbol{i} \sqrt{d}}{2 \sqrt{2}}\left[X_{n} \mid g \cdot X_{l}\right]^{\boldsymbol{i}}+\frac{\sqrt{d}}{2 \sqrt{2}}\left[X_{n} \mid g \cdot X_{l}\right]^{1} \\
& =\frac{1}{\sqrt{2}}{ }^{V} \Phi_{n, l}^{1}(g) \mp \frac{\boldsymbol{i}}{\sqrt{2}}{ }^{V} \Phi_{n, l}^{\boldsymbol{i}}(g) .
\end{aligned}
$$

Orthonormality of ${ }^{V} \Phi_{n, l}^{\bullet}$ follows from explicit formulas:

$$
{ }^{V} \Phi_{n, l}^{1}=\frac{1}{\sqrt{2}} \mathcal{V}_{+} \Psi_{n, l}+\frac{1}{\sqrt{2}} \mathcal{V}_{-} \Psi_{n, l}, \quad{ }^{V} \Phi_{n, l}^{i}=\frac{i}{\sqrt{2}} \mathcal{V}_{+} \Psi_{n, l}-\frac{i}{\sqrt{2}} \mathcal{V}_{-} \Psi_{n, l}
$$

If $\mathbb{F}=\mathbb{H}$, choose a rank 1 idempotent in $\operatorname{End}_{G}\left(V \otimes_{\mathbb{R}} \mathbb{C}\right)=\mathbb{F} \otimes_{\mathbb{R}} \mathbb{C} \cong M_{2}(\mathbb{C})$ :

$$
V \otimes_{\mathbb{R}} \mathbb{C}=\mathcal{V} \oplus \mathcal{V}^{\perp} \text { where } \mathcal{V}^{\perp} \cong \mathcal{V}, \quad \mathcal{V}=\left(V \otimes_{\mathbb{R}} \mathbb{C}\right) \boldsymbol{e}, \quad \boldsymbol{e}=\frac{1}{2}(1 \otimes 1+\boldsymbol{i} \otimes \boldsymbol{i})
$$

Now we choose $Y_{n}^{+}=\sqrt{2} X_{n} \boldsymbol{e}, Y_{n}^{-}=\sqrt{2} X_{n} \boldsymbol{j} \boldsymbol{e}$, as an orthonormal basis of $\mathcal{V}$. The calculation in the case of $\mathbb{F}=\mathbb{C}$ makes it clear that $\left\langle Y_{n}^{+} \mid Y_{n}^{+}\right\rangle=1$ and $\left\langle Y_{n}^{ \pm} \mid Y_{k}^{\bullet}\right\rangle=0$ if $n \neq k$. Let us do the remaining calculations:

$$
\begin{aligned}
\left\langle Y_{n}^{-} \mid Y_{n}^{-}\right\rangle & =\frac{1}{2}\left\langle X_{n} \boldsymbol{j} \otimes 1-X_{n} \boldsymbol{k} \otimes \boldsymbol{i} \mid X_{n} \boldsymbol{j} \otimes 1-X_{n} \boldsymbol{k} \otimes \boldsymbol{i}\right\rangle \\
& =\frac{1}{2}\left\langle X_{n} \boldsymbol{j} \mid X_{n} \boldsymbol{j}\right\rangle-\frac{\boldsymbol{i}}{2}\left\langle X_{n} \boldsymbol{j} \mid X_{n} \boldsymbol{k}\right\rangle+\frac{\boldsymbol{i}}{2}\left\langle X_{n} \boldsymbol{k} \mid X_{n} \boldsymbol{j}\right\rangle+\frac{1}{2}\left\langle X_{n} \boldsymbol{k} \mid X_{n} \boldsymbol{k}\right\rangle \\
& =\left[X_{n} \mid X_{n}\right]^{1}+\boldsymbol{i}\left[X_{n} \mid X_{n}\right]^{\boldsymbol{i}}=1, \\
\left\langle Y_{n}^{+} \mid Y_{n}^{-}\right\rangle & =\frac{1}{2}\left\langle X_{n} \otimes 1+X_{n} \boldsymbol{i} \otimes \boldsymbol{i} \mid X_{n} \boldsymbol{j} \otimes 1-X_{n} \boldsymbol{k} \otimes \boldsymbol{i}\right\rangle
\end{aligned}
$$




$$
\begin{aligned}
& =\frac{1}{2}\left\langle X_{n} \mid X_{n} \boldsymbol{j}\right\rangle-\frac{\boldsymbol{i}}{2}\left\langle X_{n} \mid X_{n} \boldsymbol{k}\right\rangle-\frac{\boldsymbol{i}}{2}\left\langle X_{n} \boldsymbol{i} \mid X_{n} \boldsymbol{j}\right\rangle-\frac{1}{2}\left\langle X_{n} \boldsymbol{i} \mid X_{n} \boldsymbol{k}\right\rangle \\
& =-\left[X_{n} \mid X_{n}\right]^{\boldsymbol{j}}+\boldsymbol{i}\left[X_{n} \mid X_{n}\right]^{\boldsymbol{k}}=0 .
\end{aligned}
$$

Let us optimise the notation by $\Psi_{n, l}^{ \pm ?}={ }^{\mathcal{V}} \Psi_{n^{ \pm}, l^{\text {? }}}$. The calculation

$$
\Psi_{n, l}^{++}=\frac{1}{\sqrt{2}} V \Phi_{n, l}^{1}(g)-\frac{\boldsymbol{i}}{\sqrt{2}}^{V} \Phi_{n, l}^{i}(g)
$$

is as in the complex case. Again $d=2 \operatorname{dim}_{\mathbb{C}} \mathcal{V}$. Let us calculate the remaining functions.

$$
\begin{aligned}
& \Psi_{n, l}^{--}(g)=\frac{\sqrt{d}}{2 \sqrt{2}}\left\langle X_{n} \boldsymbol{j} \otimes 1-X_{n} \boldsymbol{k} \otimes \boldsymbol{i} \mid g \cdot X_{l} \boldsymbol{j} \otimes 1-g \cdot X_{l} \boldsymbol{k} \otimes \boldsymbol{i}\right\rangle \\
& =\frac{\sqrt{d}}{2 \sqrt{2}}\left\langle X_{n} \boldsymbol{j} \mid g \cdot X_{l} \boldsymbol{j}\right\rangle-\frac{\boldsymbol{i} \sqrt{d}}{2 \sqrt{2}}\left\langle X_{n} \boldsymbol{j} \mid g \cdot X_{l} \boldsymbol{k}\right\rangle+\frac{\boldsymbol{i} \sqrt{d}}{2 \sqrt{2}}\left\langle X_{n} \boldsymbol{k} \mid g \cdot X_{l} \boldsymbol{j}\right\rangle \\
& +\frac{\sqrt{d}}{2 \sqrt{2}}\left\langle X_{n} \boldsymbol{k} \mid g \cdot X_{l} \boldsymbol{k}\right\rangle \\
& =\frac{1}{\sqrt{2}}{ }^{V} \Phi_{n, l}^{1}(g)+\frac{i}{\sqrt{2}}^{V} \Phi_{n, l}^{i}(g), \\
& \Psi_{n, l}^{+-}(g)=\frac{\sqrt{d}}{2 \sqrt{2}}\left\langle X_{n} \otimes 1+X_{n} \boldsymbol{i} \otimes \boldsymbol{i} \mid g \cdot X_{l} \boldsymbol{j} \otimes 1-g \cdot X_{l} \boldsymbol{k} \otimes \boldsymbol{i}\right\rangle \\
& =\frac{\sqrt{d}}{2 \sqrt{2}}\left\langle X_{n} \mid g \cdot X_{l} \boldsymbol{j}\right\rangle-\frac{\boldsymbol{i} \sqrt{d}}{2 \sqrt{2}}\left\langle X_{n} \mid g \cdot X_{l} \boldsymbol{k}\right\rangle-\frac{\boldsymbol{i} \sqrt{d}}{2 \sqrt{2}}\left\langle X_{n} \boldsymbol{i} \mid g \cdot X_{l} \boldsymbol{j}\right\rangle \\
& -\frac{\sqrt{d}}{2 \sqrt{2}}\left\langle X_{n} \boldsymbol{i} \mid g \cdot X_{l} \boldsymbol{k}\right\rangle \\
& =\frac{-1}{\sqrt{2}} V \Phi_{n, l}^{j}(g)+\frac{i}{\sqrt{2}} V \Phi_{n, l}^{k}(g), \\
& \Psi_{n, l}^{-+}(g)=\frac{\sqrt{d}}{2 \sqrt{2}}\left\langle X_{n} \boldsymbol{j} \otimes 1-X_{n} \boldsymbol{k} \otimes \boldsymbol{i} \mid g \cdot X_{l} \otimes 1+g \cdot X_{l} \boldsymbol{i} \otimes \boldsymbol{i}\right\rangle \\
& =\frac{\sqrt{d}}{2 \sqrt{2}}\left\langle X_{n} \boldsymbol{j} \mid g \cdot X_{l}\right\rangle+\frac{\boldsymbol{i} \sqrt{d}}{2 \sqrt{2}}\left\langle X_{n} \boldsymbol{j} \mid g \cdot X_{l} \boldsymbol{i}\right\rangle+\frac{\boldsymbol{i} \sqrt{d}}{2 \sqrt{2}}\left\langle X_{n} \boldsymbol{k} \mid g \cdot X_{l}\right\rangle \\
& -\frac{\sqrt{d}}{2 \sqrt{2}}\left\langle X_{n} \boldsymbol{k} \mid g \cdot X_{l} \boldsymbol{i}\right\rangle \\
& =\frac{1}{\sqrt{2}} V \Phi_{n, l}^{j}(g)+\frac{i}{\sqrt{2}} V \Phi_{n, l}^{k}(g) .
\end{aligned}
$$

Orthonormality of $V_{n, l}^{\bullet}$ follows from explicit formulas:

$$
\begin{aligned}
& V \Phi_{n, l}^{1}=\frac{1}{\sqrt{2}} \Psi_{n, l}^{++}+\frac{1}{\sqrt{2}} \Psi_{n, l}^{--}, \quad V \Phi_{n, l}^{i}=\frac{i}{\sqrt{2}} \Psi_{n, l}^{++}-\frac{i}{\sqrt{2}} \Psi_{n, l}^{--}, \\
& { }^{V} \Phi_{n, l}^{j}=\frac{1}{\sqrt{2}} \Psi_{n, l}^{-+}-\frac{1}{\sqrt{2}} \Psi_{n, l}^{+-},
\end{aligned}
$$




\section{References}

[1] M. Arnaudon, Semi-martingales dans les espaces homogènes, Ann. Inst. H. Poincaré Probab. Statist., 29 (1993), 269-288.

[ 2 ] V. I. Arnol'd, Mathematical methods of classical mechanics, Springer-Verlag, New York, 1989.

[3] L. Bérard-Bergery and J.-P. Bourguignon, Laplacians and Riemannian submersions with totally geodesic fibres, Illinois J. Math., 26 (1982), 181-200.

[ 4] M. Berger, Sur les groupes d'holonomie homogène des variétés à connexion affine et des variétés riemanniennes, Bull. Soc. Math. France, 83 (1955), 279-330.

[5] N. Berline, E. Getzler and M. Vergne, Heat kernels and Dirac operators, Springer-Verlag, 1992.

[6] J. Berndt and L. Vanhecke, Geometry of weakly symmetric spaces, J. Math. Soc. Japan, 48 (1996), 745-760.

[ 7 ] A. L. Besse, Einstein manifolds, 10, Springer-Verlag, Berlin, 1987.

[ 8 ] J.-M. Bismut, The hypoelliptic Laplacian on a compact Lie group, J. Funct. Anal., 255 (2008), 2190-2232.

[ 9 ] J.-M. Bismut, Hypoelliptic Laplacian and orbital integrals, Ann. of Math. Studies, 177, Princeton University Press, Princeton, NJ, 2011.

[10] J.-M. Bismut and G. Lebeau, Laplacien hypoelliptique et torsion analytique, C. R. Math. Acad. Sci. Paris, 341 (2005), 113-118.

[11] A. N. Borodin and M. I. Freidlin, Fast oscillating random perturbations of dynamical systems with conservation laws, Ann. Inst. H. Poincaré Probab. Statist., 31 (1995), 485-525.

[12] F. E. Burstall and J. H. Rawnsley, Twistor theory for Riemannian symmetric spaces, LNIM, 1424, Springer-Verlag, 1990.

[13] J. Cheeger and M. Gromov, Collapsing Riemannian manifolds while keeping their curvature bounded, I, J. Differential Geom., 23 (1986), 309-346.

[14] R. Darling, Martingale on manifolds and geometric ito calculus, PhD thesis, The University of Warwick.

[15] R. M. Dowell, Differentiable Approximations to Brownian Motion on Manifolds, PhD thesis, University of Warwick, 1980.

[16] E. B. Dynkin, Markov processes, I, II, (Translated with the authorization and assistance of the author by J. Fabius, V. Greenberg, A. Maitra, G. Majone), Die Grundlehren der Mathematischen Wissenschaften, Bände 121, 122, Academic Press Inc., Publishers, Springer-Verlag, 1965.

[17] J. Eells and K. D. Elworthy, Stochastic dynamical systems, In: Control theory and topics in functional analysis (Internat. Sem., Internat. Centre Theoret. Phys., Trieste, 1974), III, Internat. Atomic Energy Agency, Vienna, 1976, 179-185.

[18] K. D. Elworthy, Stochastic differential equations on manifolds, London Math. Soc. Lecture Note Series, 70, Cambridge University Press, Cambridge, 1982.

[19] K. D. Elworthy, Y. Le Jan and X.-M. Li, On the geometry of diffusion operators and stochastic flows, Lecture Notes in Math., 1720, Springer-Verlag, 1999.

[20] K. D. Elworthy, Y. Le Jan and X.-M. Li, The geometry of filtering, Frontiers in Mathematics, Birkhäuser Verlag, Basel, 2010.

[21] M. Émery, Stochastic calculus in manifolds, Universitext, Springer-Verlag, Berlin, 1989.

[22] M. Freidlin, Markov processes and differential equations: asymptotic problems, Lectures in Math., ETH Zürich, Birkhäuser Verlag, Basel, 1996.

[23] M. Freidlin and M. Weber, On stochasticity of solutions of differential equations with a small delay, Stoch. Dyn., 5 (2005), 475-486.

[24] M. I. Freidlin and A. D. Wentzell, Diffusion processes on graphs and the averaging principle, Ann. Probab., 21 (1993), 2215-2245.

[25] K. Fukaya, Collapsing of Riemannian manifolds and eigenvalues of Laplace operator, Invent. Math., 87 (1987), 517-547.

[26] I. I. Gonzales-Gargate and P. R. Ruffino, An averaging principle for diffusions in foliated spaces, Ann. Probab., 44 (2016), 567-588.

[27] V. V. Gorbatsevich, A. L. Onishchik and E. B. Vinberg, Foundations of Lie theory and Lie transformation groups, Springer-Verlag, Berlin, 1997. 
[28] R. Z. Has'minskii, Stochastic processes defined by differential equations with a small parameter, Teor. Verojatnost. i Primenen, 11 (1966), 240-259.

[29] E. Heintze and W. Ziller, Isotropy irreducible spaces and s-representations, Differential Geom. Appl., 6 (1996), 181-188.

[30] S. Helgason, Differential geometry and symmetric spaces, Academic Press, New York-London, 1962.

[31] M. Högele and P. Ruffino, Averaging along foliated Lévy diffusions, Nonlinear Anal., 112 (2015), 1-14.

[32] D. Holm, J. Marsden, T. Ratiu and A. Weinstein, Stability of rigid body motion using the energy-Casimir method, In: Fluids and plasmas: geometry and dynamics, Amer. Math. Soc., 1984, 15-23.

[33] L. Hörmander, Hypoelliptic second order differential equations, Acta Math., 119 (1967), $147-171$.

[34] L. Hörmander, The analysis of linear partial differential operators, I, Distribution theory and Fourier analysis, 256, Springer-Verlag, Berlin, second edition, 1990.

[35] N. Ikeda and Y. Ogura, A degenerating sequence of Riemannian metrics on a manifold and their Brownian motions, In: Diffusion processes and related problems in analysis, I, Progr. Probab., 22, Birkhäuser Boston, 1990, 293-312.

[36] N. Ikeda and S. Watanabe, Stochastic differential equations and diffusion processes, North-Holland Publishing Co., second edition, 1989.

[37] N. Iwahori, On real irreducible representations of Lie algebras, Nagoya Math. J., 14 (1959), 59-83.

[38] A. Kasue and H. Kumura, Spectral convergence of Riemannian manifolds, Tohoku Math. J. (2), 46 (1994), 147-179.

[39] S. Kobayashi, Transformation groups in differential geometry, Classics in Mathematics. SpringerVerlag, Berlin, 1995. Reprint of the 1972 edition.

[40] S. Kobayashi and K. Nomizu, Foundations of differential geometry, I, Interscience Publishers, a division of John Wiley \& Sons, New York-London, 1963.

[41] H. A. Kramers, Brownian motion in a field of force and the diffusion model of chemical reactions, Physica, 7 (1940), 284-304.

[42] T. G. Kurtz, A general theorem on the convergence of operator semigroups, Trans. Amer. Math. Soc., 148 (1970), 23-32.

[43] J.-A. Lázaro-Camí and J.-P. Ortega, Reduction, reconstruction, and skew-product decomposition of symmetric stochastic differential equations, Stoch. Dyn., 9 (2009), 1-46.

[44] X.-M. Li, An averaging principle for a completely integrable stochastic Hamiltonian system, Nonlinearity, 21 (2008), 803-822.

[45] X.-M. Li, Effective diffusions with intertwined structures, arxiv:1204.3250, 2012.

[46] X.-M. Li, Random perturbation to the geodesic equation, Annals of Probability, 44 (2015), 544566.

[47] X.-M. Li, Limits of random differential equations on manifolds, Probab. Theory Relat. Fields, 166 (2016), 659-712.

[48] X.-M. Li, Perturbation of conservation laws and averaging on manifolds, to appear in Abelsymposium 2016, 2017.

[49] M. Liao and L. Wang, Average under the Iwasawa transformation, Proc. Amer. Math. Soc., 135 (2007), 895-901.

[50] P. Malliavin, Géométrie différentielle stochastique, Séminaire de Mathématiques Supérieures 64, Presses de l'Université de Montréal, 1978, Notes prepared by Danièle Dehen and Dominique Michel.

[51] J. E. Marsden, G. Misiołek, J.-P. Ortega, M. Perlmutter and T. S. Ratiu, Hamiltonian reduction by stages, Lecture Notes in Math., 1913, Springer, Berlin, 2007.

[52] R. R. Mazzeo and R. B. Melrose, The adiabatic limit, Hodge cohomology and Leray's spectral sequence for a fibration, J. Differential Geom., 31 (1990), 185-213.

[53] J. Milnor, Curvatures of left invariant metrics on Lie groups, Advances in Math., 21 (1976), 293-329.

[54] D. Montgomery and H. Samelson, Transformation groups of spheres, Ann. of Math. (2), 44 (1943), $454-470$. 
[55] S. B. Myers and N. E. Steenrod, The group of isometries of a Riemannian manifold, Ann. of Math. (2), 40 (1939), 400-416.

[56] E. Nelson, Dynamical theories of Brownian motion, Princeton University Press, Princeton, NJ, 1967.

[57] K. Nomizu, Invariant affine connections on homogeneous spaces, Amer. J. Math., 76 (1954), 33-65.

[58] M. Obata, On subgroups of the orthogonal group, Trans. Amer. Math. Soc., 87 (1958), 347-358.

[59] Y. Ogura and S. Taniguchi, A probabilistic scheme for collapse of metrics, J. Math. Kyoto Univ., 36 (1996), 73-92.

[60] G. C. Papanicolaou and W. Kohler, Asymptotic theory of mixing stochastic ordinary differential equations, Comm. Pure Appl. Math., 27 (1974), 641-668.

[61] G. C. Papanicolaou and S. R. S. Varadhan, A limit theorem with strong mixing in Banach space and two applications to stochastic differential equations, Comm. Pure Appl. Math., 26 (1973), 497-524.

[62] M. Rosenblatt, Markov processes, Structure and asymptotic behavior, Die Grundlehren der mathematischen Wissenschaften, Band 184, Springer-Verlag, Heidelberg, 1971.

[63] A. Selberg, Harmonic analysis and discontinuous groups in weakly symmetric Riemannian spaces with applications to Dirichlet series, J. Indian Math. Soc. (N.S.), 20 (1956), 47-87.

[64] I. Shigekawa, On stochastic horizontal lifts, Z. Wahrsch. Verw. Gebiete, 59 (1982), 211-221.

[65] J. Simons, On the transitivity of holonomy systems, Ann. of Math. (2), 76 (1962), 213-234.

[66] R. L. Stratonovich, A limit theorem for solutions of differential equations with random right-hand side, Theory Prob. Appl., 11, 1960.

[67] S. Tanno, The first eigenvalue of the Laplacian on spheres, Tôhoku Math. J. (2), 31 (1979), 179-185.

[68] H. Urakawa, The first eigenvalue of the Laplacian for a positively curved homogeneous Riemannian manifold, Compositio Math., 59 (1986), 57-71.

[69] J. A. Wolf, The geometry and structure of isotropy irreducible homogeneous spaces, Acta Math., 120 (1968), 59-148.

[70] A. Barut and R. Raczka, Theory of group representations and applications, PWN-Polish Scientific Publishers, Warsaw, 1977.

[71] E. Hewitt and K. Ross, Abstract harmonic analysis, volumes I and II, Springer-Verlag, Berlin-New York, 1979.

\author{
Xue-Mei LI \\ Department of Mathematics \\ Imperial College London \\ London SW7 2AZ, UK \\ E-mail: xue-mei.li@imperial.ac.uk
}

\author{
Dmitriy RumYNIN \\ Department of Mathematics \\ University of Warwick \\ Coventry, CV4 7AL, UK \\ E-mail: D.Rumynin@warwick.ac.uk
}

